\title{
THE SPECTRAL THEORY OF BOUNDED FUNCTIONS
}

BX

\author{
CARL S. HERZ(1)
}

The present article is intended as a survey of the title subject. The material is closely intertwined with all branches of harmonic analysis. Historically, the rigorous foundations of the theory arise in Riemann's treatment of trigonometric series, and spectral theory is essentially equivalent to the study of formal multiplication. The original motivation for the modern treatment, due to Wiener, Carleman, and Beurling, came from the study of integral equations with convolution kernels. There are applications to linear partial differential equations with constant coefficients, and there is a very close connection with problems about entire functions bounded on a line. My own concern with the topic commenced with questions about the theory of approximation for multiple Fourier transforms.

When I first looked at the subject the basic material did not appear very well organized, and certain elementary facts were not recognized as immediately obvious to experts in the field. Thus, in 1954, I set about to record what was known with certain useful additions. Shortly after the original version of this work was finished in 1956, the paper of Domar appeared. (An author's name in small capitals indicates a reference to the bibliography.) There was a considerable overlap, for, although the two papers were quite independent, both were heavily influenced by unpublished notes of Beurling. I have revised the paper in an attempt to suppress details which may be found elsewhere. Also, I have taken advantage of more recent work by others and myself to improve the material of the last half of the paper.

After a preliminary section introducing much of the notation and basic definitions, the contents of this paper are divided into six parts. The headings are:

1. The spectrum.

2. The point spectrum.

3. Potential theory and spectral analysis.

4. The spectral synthesis problem.

5. Representations.

6. Examples of spectral synthesis.

Received by the editors May 18, 1956 and, in revised form, January 28, 1959.

(1) This research was supported in part by the United States Air Force through the Air Force Office of Scientific Research of the Air Research and Development Command, under Contracts No. AF18(600)-685 and No. AF18(600)-1109. Reproduction in whole or in part is permitted for any purpose of the United States Government. 
The first section introduces the fundamental concepts and contains a rough outline of the material to be treated in the later portions. After it, $\S \S 2$ and 3 may be read independently of the rest of the paper.

The exposition here is carried out on a locally compact abelian group. Important ideas, when first introduced, are generally discussed quite fully with the viewpoint of classical analysis in mind, and examples are given, usually at the end of a section, to illustrate what is going on.

0 . Preliminaries. Let $\Gamma$ be a locally compact Hausdorff space. We denote by $C(\Gamma)$ the linear space of bounded continuous functions on $\Gamma$; for $\varphi \in C(\Gamma)$, $\|\varphi\|$ is defined as sup $|\varphi(x)|$, where the supremum is taken over all $x \in \Gamma$. $C(\Gamma)$ has a subspace $C_{0}(\Gamma)$ consisting of the functions which vanish at $\infty$, i.e. $\varphi \in C_{0}(\Gamma)$ if $\varphi$ is continuous and given $\epsilon>0$ there is a compact subset $\mathrm{K} \subset \Gamma$ such that $|\varphi(x)|<\epsilon$ for $x \in \tilde{\mathrm{K}}$, the complement of $\mathrm{K}$. The dual Banach space to $C_{0}(\Gamma)$ is $B(\Gamma)$, the space of bounded Radon measures. For $\mu \in B(\Gamma)$, $\|\mu\|=\int|\mu(d x)|$, the total variation.

For our purposes the most useful topology on $C(\Gamma)$ is the strict topology $\left({ }^{2}\right)$ which may be defined in either of two equivalent ways. The first is this: consider $C(\Gamma)$ operating on $C_{0}(\Gamma)$ by pointwise multiplication; the strict topology is the strong operator topology. For the alternative description we shall define the neighborhoods of 0 . Let $\left\{\epsilon_{n}, K_{n}\right\}$ be a sequence of pairs of positive numbers $\epsilon_{n}$ and compact sets $\mathrm{K}_{n} \subset \Gamma$ such that $\epsilon_{n} \nearrow \infty$ and $\mathrm{K}_{n} \subset \mathrm{K}_{n+1}$. A strict neighborhood of 0 in $C(\Gamma)$ consists of all functions $\varphi \in C(\Gamma)$ satisfying the inequalities $|\varphi(x)|<\epsilon_{n}$ for $x \in \mathrm{K}_{n}$. The strict topology is a locally convex topology in which $C(\Gamma)$ is a complete topological linear space. The space of strictly continuous linear functionals on $C(\Gamma)$ admits a natural identification with $B(\Gamma)$. On norm-bounded sets, the strict topology agrees with the one given by uniform convergence on compact sets.

Suppose $\mathrm{P}$ is another locally compact Hausdorff space and $\rho: \Gamma \rightarrow \mathrm{P}$ a continuous map. Given $\varphi \in C(\mathrm{P})$ we define $\hat{\rho} \varphi$ as a function on $\Gamma$ by $\hat{\rho} \varphi(x)$ $=\varphi(\rho x)$. (The reason for the notation will become clear in a little while.) It is obvious that one has a map $\hat{\rho}: C(\mathrm{P}) \rightarrow C(\Gamma)$ and that $\|\hat{\rho} \varphi\| \leqq\|\varphi\|$. What is of paramount importance is that $\hat{\rho}$ is continuous with respect to the strict topologies. Here the second definition of the strict topology is most useful; the neighborhood of 0 in $C(\Gamma)$ corresponding to the sequence $\left\{\epsilon_{n}, \mathrm{~K}_{n}\right\}$ contains the image of the neighborhood of 0 in $C(\mathrm{P})$ corresponding to the sequence $\left\{\epsilon_{n}, \rho \mathrm{K}_{n}\right\}$. The dual of the map $\hat{\rho}: C(\mathrm{P}) \rightarrow C(\Gamma)$ is a map which we denote again by $\rho ; \rho: B(\Gamma) \rightarrow B(\mathrm{P})$. If $\mu \in B(\Gamma)$, the measure $\rho \mu \in B(\mathrm{P})$ is defined on compact $\mathrm{K} \subset \mathrm{P}$ by $\rho \mu(\mathrm{K})=\mu\left(\rho^{-1} \mathrm{~K}\right)$. At the beginning of $\S 5$ we shall return to the examination of these maps.

We are mainly concerned with the case where $\Gamma$ is a locally compact abelian group. Throughout this paper the word "group" is used to mean

(2) The strict topology has been used by Beurling in unpublished work and is discussed in Buck (where it is called " $\beta$ "). 
only "locally compact abelian group." The group operation is written as addition. If $P$ is another group, a map $\rho: \Gamma \rightarrow P$ is called a "representation" if it is continuous and is a homomorphism in the algebraic sense. The term "homomorphism" is reserved for representations in which the image set $\rho \Gamma$ is closed and the image of an open set in $\Gamma$ is relatively open in $\rho \Gamma$. In particular, a subset $\Delta$ of $\Gamma$ is called a "subgroup" if and only if the identity map $\iota: \Delta \rightarrow \Gamma$ is an injective homomorphism of groups.

The topology of a group may be described by giving a basis for the neighborhoods of 0 . We use the term "nucleus" to denote an open neighborhood of 0 with compact closure which satisfies one additional condition. This extra condition is needed only in a few places; it is that we require nuclei to be Jordan measurable. A subset of a group is said to be "Jordan measurable" if its boundary has Haar measure zero. One can always choose a fundamental system of neighborhoods of 0 to be nuclei. To see this, we note that the topology of a group may be described by a system of continuous invariant pseudometrics $\left({ }^{2 a}\right)$. An invariant pseudometric on $\Gamma$ is a real valued function $\delta$ with the properties $\delta(x) \geqq \delta(0)=0, \delta(-x)=\delta(x)$, and $\delta(x+y) \leqq \delta(x)+\delta(y)$. Then $\delta(x-y)$ has all the properties of a distance except that $\delta(x-y)=0$ need not imply $x=y$. It is well known that the topology of a group can be described by a system $\{\delta\}$ of continuous invariant pseudometrics where $\delta(x)=0$ for each $\delta$ of the system implies $x=0$ and where for each $\delta$, the sets $\{x \mid \delta(x) \leqq \epsilon\}$ are compact for sufficiently small $\epsilon$. Thus a fundamental system of neighborhoods of 0 in $\Gamma$ may be given by the sets $\mathrm{N}(\delta, \xi)=\{x \mid \delta(x)<\xi\}$ where $\delta$ runs through an admissible system of continuous invariant pseudometrics and for each $\delta, \xi$ runs through a set of positive numbers tending to 0 . Holding $\delta$ fixed, set $\alpha(\xi)=|\mathbf{N}(\delta, \zeta)|$ where $|\mathbf{A}|$ denotes the Haar measure of a set $\left(^{3}\right) \mathrm{A}$. Let $\epsilon$ be such that $\{x \mid \delta(x) \leqq \epsilon\}$ is compact. The function $\alpha$ is increasing on the interval $[0, \epsilon]$. Hence it has at most a countable set of discontinuities. For each $\xi$ in the open interval $(0, \epsilon)$ which is a point of right-continuity for $\alpha, \mathrm{N}(\delta, \xi)$ is a nucleus. More generally, we shall have occasion to consider sets $A$ with the properties that $0 \in \operatorname{Int} A$, the interior of $A$; the closure $\bar{A}$, of $A$, is compact, and A is Jordan measurable. Such an A will be called a $J$-set.

It is assumed that the reader is familiar with the theory of Fourier analysis on groups as exposed, say, in CARTAN and Godement. It will be necessary to explain the notation and conventions used in the present paper. If $t$ is a point of the character group $\hat{\Gamma}$ of $\Gamma$, the character itself is denoted by $e_{t}$ and we write $e_{t}(x)=e(t x)$. The topology of $\hat{\Gamma}$ is equivalent to that inherited by the set of functions $\left\{e_{t} \mid t \in \hat{\Gamma}\right\}$ as a subset of $C(\Gamma)$ in the strict topology. Thus whenever $\rho: \Gamma \rightarrow \mathrm{P}$ is a representation, the previously defined map $\hat{\rho}$ agrees with the natural dual representation on the character groups, $\hat{\rho}: \hat{\mathrm{P}} \rightarrow \hat{\mathrm{C}}$. For

(2a) See KELLEY, p. 210.

(3) The notation ${ }^{\alpha}|x|$ " where $x$ is a point is used to denote the length of a vector in Euclidean space or some appropriate analogue. 
measures $\mu \in B(\Gamma)$, the Fourier-Stieltjes transform $\hat{\mu}$ is defined by $\hat{\mu}(t)$ $=\int \bar{e}(t x) \mu(d x)$, the bar denoting complex conjugation. More generally, $\mu$ considered as a linear functional operating on $\varphi \in C(\Gamma)$ is associated with the corresponding measure by $(\varphi, \mu)=\int \varphi(-x) \mu(d x)$. Thus $\hat{\mu}(t)=\left(e_{t}, \mu\right)$. For a function $\varphi$ defined on $\Gamma$ and a point $x \in \Gamma$ we define $\varphi^{x}$ by $\varphi^{x}(y)=\varphi(x+y)$. For $\varphi \in C(\Gamma)$ and $\mu \in B(\Gamma)$ the convolution $\mu \circ \varphi$ is the element of $C(\Gamma)$ defined by $\mu \circ \varphi(x)=\left(\varphi^{x}, \mu\right)=\int \varphi(x-y) \mu(d y)$. We shall employ the notation $L \circ \varphi$ for any linear transformation, $L$, of functions which commutes with translation. The translation operators themselves may be represented by measures $\epsilon_{x} \in B(\Gamma)$ where $\epsilon_{x}$ is the measure of mass 1 concentrated at the point $x$; $\epsilon=\epsilon_{0}$ is the unit measure at the identity. The convolution $\mu \circ \nu$ of two measures $\mu, \nu \in B(\Gamma)$ can be defined in several equivalent ways. As a linear functional on $C(\Gamma), \mu \circ \nu$ may be described by saying that $(\varphi, \mu \circ \nu)$ is the common value of $(\mu \circ \varphi, \nu)$ and $(\nu \circ \varphi, \mu)$. Alternatively, for a compact set $K \subset \Gamma$ we can write $\mu \circ \nu(\mathrm{K})=\int \nu(\mathrm{K}-x) \mu(d x)$ since for fixed $\mathrm{K}, \nu(\mathrm{K}-x)$ is a linear combination of semi-continuous functions.

Associated with a measure $\mu$ is a closed set $\Sigma(\mu)$ called "the spectrum" or "the support" of $\mu . y \in \Sigma(\mu)$ if and only if $\int_{\Omega}|\mu(d x)|>0$ for each open set $\Omega$ containing $y . \Sigma_{p}(\mu)$ is the subset of $\Sigma(\mu)$ consisting of those points to which $\mu$ assigns a nonzero measure. We shall also employ the notation $\mathrm{Z}(\mu)$ $=\{t \mid \hat{\mu}(t)=0\}$ for the set of zeros of the Fourier transform.

On a group, $C(\Gamma)$ has a subspace $C_{u}(\Gamma)$ consisting of the uniformly continuous functions. Among these are the Fourier-Stieltjes transforms. Here arises a slight asymmetry, $-\varphi \in C_{u}(\Gamma)$ is the Fourier-Stieltjes transform of $\alpha \in B(\hat{\Gamma})$ if $\varphi(x)=\int e(t x) \alpha(d t)$. Also, on a group, one has a Haar measure. Integration with respect to some fixed normalization of the Haar measure is indicated by the usual calculus notation $d x$. One may consider the usual function spaces $L^{p}(\Gamma), 1 \leqq p \leqq \infty$, defined with respect to the Haar measure. At this point a certain notational difficulty arises. The elements of the Banach spaces $L^{p}(\Gamma)$ may be identified with certain equivalence classes of functions. However it will be convenient to use the notation $f \in L^{p}, p<\infty$, to mean that $f$ is a definite, everywhere defined, Baire function which vanishes outside a $\sigma$-compact subset of $\Gamma$ and for which $\|f\|_{p}=\left\{\int|f(x)|^{p} d x\right\}^{1 / p}$ $<\infty$. Some previous conventions will be taken over. If $f \in L^{p}(\Gamma)$ and $g \in L^{q}(\Gamma)$ where $1 / p+1 / q=1$ we write $(f, g)=\int f(-x) g(x) d x$ and $f \circ g$ for the convolution, which is an element of $C_{u}(\Gamma)$. The ordinary Hilbert space inner product of $f, g \in L^{2}(\Gamma)$ is $\left(f, g^{*}\right)$ where, in general, $\varphi^{*}$ denotes the function $\varphi^{*}(x)$ $=\bar{\varphi}(-x)$.

An element $f \in L^{1}(\Gamma)$ is naturally associated with an element $\mu \in B(\Gamma)$ where $\mu(d x)=f(x) d x$. All the previous notations involving $\mu$ are carried over to $f$, e.g. $\hat{f}(t)=\int \bar{e}(t x) f(x) d x$. However, if also $\hat{f} \in L^{1}(\hat{\Gamma})$, we tacitly assume that the Fourier inversion formula $f(x)=\int e(t x) \hat{f}(t) d t$ holds everywhere. This convention is adopted whenever a pair of functions $g$ and $g$, the former defined 
on $\Gamma$, the latter on $\hat{\Gamma}$, is written down and one of them is absolutely integrable.

We plan to do harmonic analysis in the space $C(\Gamma)$ with the strict topology, whereas it is often carried out in $L^{\infty}(\Gamma)$ with the $w^{*}$-topology as dual space to $L^{1}(\Gamma)$. The difference is in most cases a trivial one. $C(\Gamma)$ may be considered as imbedded in $L^{\infty}(\Gamma)$. The relative $w^{*}$-topology on $C(\Gamma)$ is the weak topology obtained by using only the absolutely continuous measures, i.e. $L^{1}(\Gamma)$, instead of all of $B(\Gamma)$, as linear functionals. However, for our purposes the relative $w^{*}$-topology is only apparently weaker than the strict topology.

LEMma. Let $E$ be a strictly-closed, translation invariant, convex subset of $C(\Gamma)$. Then $E$ is closed in the relative $w^{*}$-topology.

Proof. Suppose $\varphi \notin E$. Let $N$ be a nucleus in $\Gamma$ and set $f_{N}(x)=|N|^{-1}$ for $x \in \mathrm{N}, f(x)=0$ for $x \notin N$. Now if $\varphi \in C(\Gamma), \lim _{\mathrm{N} \rightarrow 0} f_{\mathrm{N}} \circ \varphi=\varphi$ where the limit is taken in the strict topology. Thus for some $\mathrm{N}, f_{\mathrm{N}} \circ \varphi \notin E$ which means that there is an $f \in L^{1}(\Gamma)$ with $\|f\|_{1}=1$ and $f \geqq 0$ such that $f \circ \varphi \notin E$. Using all the hypotheses on $E$ one sees that $f \circ E \subset E$. Since $f \circ \varphi \notin E$ there is a hyperplane determined by a $\mu \in B(\Gamma)$ separating $f \circ \varphi$ from $E$ and a fortiori from $f \circ E$. Since $(f \circ \psi, \mu)=(\psi, f \circ \mu)$ it follows that $f \circ \mu$ determines a hyperplane separating $\varphi$ from $E$, but $f \circ \mu \in L^{1}(\Gamma)$. Hence $\varphi$ does not belong to the relative $w^{*}$-closure of $E$.

In BEURLING [1] there is a notion of convergence used for harmonic analysis which is stronger than strict convergence. Let $F$ be a subset of $C(\Gamma)$. One says that $\varphi \in C(\Gamma)$ is the "narrow limit" of functions in $F$ if for each pair $(\epsilon, \mathrm{K})$ consisting of a number $\epsilon>0$ and a compact set $\mathrm{K} \subset \Gamma$ there is a $\psi \in F$ such that $\|\psi\|<\|\varphi\|+\epsilon$ and $|\varphi(x)-\psi(x)|<\epsilon$ for $x \in \mathbf{K}$.

If $A$ and $B$ are two subsets of a group $\Gamma$ we define $A+B=\{x+y \mid x \in A, y \in B\}$. The notation $\mathbf{A} \oplus \mathbf{B}$ is used when the representation of a point in $A+B$ as a sum is unique. In particular, the direct sum of two groups $\Gamma_{1}$ and $\Gamma_{2}$ is written as $\Gamma_{1} \oplus \Gamma_{2}$. The groups of primary interest to us are $R^{r}$ and $I^{r}$, the respective $r$-fold direct sums of the reals and the integers. The index $r$ here is a special case of the notion of rank. A group $\Gamma$ is of rank 0 if each element belongs to a compact subgroup; $\Gamma$ is of rank $r, 1 \leqq r<\infty$, if $\Gamma$ has a subgroup $\Delta$ isomorphic to $I$ such that $\Gamma / \Delta$ is of rank $r-1$; otherwise the rank is infinite. The rank equals the dimension of the character group for groups satisfying the second axiom of countability. In the case of discrete groups, the rank is the maximum number of linearly independent elements. The terminology used here is not standard, but the rank, as defined above, seems to be the most important structural parameter for the needs of this work. The reader is referred to WEIL for important facts about the structure of groups.

1. The spectrum. Suppose $\varphi \in C(\Gamma)$ is a Fourier-Stieltjes transform, i.e. $\varphi(x)=\int e(t x) \alpha(d t)$ where $\alpha \in B(\hat{\Gamma})$. The spectrum of $\varphi, \Lambda(\varphi)$, is defined to be the set $\Sigma(\alpha)$, the spectrum (support) of $\alpha$, and the point spectrum, $\Lambda_{p}(\varphi)$, is 
$\Sigma_{p}(\alpha)$. The first matter we shall take up is the problem of extending the notion of spectrum to all of $C(\Gamma)$. In $\$ 2$, the analogous problem for the point spectrum is discussed.

Given $\varphi$ as above and $\mu \in B(\Gamma), \mu \circ \varphi(x)=\int e(t x) \hat{\mu}(t) \alpha(d t)$. It follows from the uniqueness of Fourier-Stieltjes transforms that $\mu \circ \varphi=0$ if and only if $\Sigma(\alpha)=\Lambda(\varphi) \subset \mathrm{Z}(\mu)$, the set of zeros of $\hat{\mu}$. It is also clear that if $t \notin \Lambda(\varphi)$ there is a $\mu \in B(\Gamma)$ such that $\Lambda(\varphi) \subset \mathrm{Z}(\mu)$ while $\hat{\mu}(t) \neq 0\left({ }^{3 \mathrm{a}}\right)$. We are prompted to make a formal definition.

Definition. For $\varphi \in C(\Gamma)$, the spectrum of $\varphi$ is the set $\Lambda(\varphi)=\cap \mathrm{Z}(\mu)$ where the intersection is taken over all $\mu \in B(\Gamma)$ such that $\mu \circ \varphi=0$.

It is appropriate to record here some basic results; a good reference is Godement. First there is the dual form of Wiener's Tauberian Theorem.

1.1. Theorem. For $\varphi \in C(\Gamma), \Lambda(\varphi)=\varnothing$ if and only if $\varphi=0$.

For a given $\varphi \in C(\Gamma)$, let $E(\varphi)$ be the smallest, strictly closed, translation invariant subspace of $C(\Gamma)$ which contains $\varphi$. An alternative definition of the spectrum is provided by the next statement.

1.2. TheOREM. Given $\varphi \in C(\Gamma)$, the statements $t \in \Lambda(\varphi)$ and $e_{t} \in E(\varphi)$ are equivalent.

Given a subset $\mathrm{T}$ of $\hat{\Gamma}$, we denote by $E^{\prime}(\mathrm{T})$ the smallest strictly closed subspace of $C(\Gamma)$ containing those functions $\varphi$ with $\Lambda(\varphi) \subset \mathrm{T} . E(\mathrm{~T})$ is similarly defined as the smallest strictly closed subspace of $C(\Gamma)$ containing the functions $e_{t}$ where $t \in \mathrm{T}$. Obviously we have $E(\mathrm{~T}) \subset E^{\prime}(\mathrm{T})$. The question whether one has $E(\mathrm{~T})=E^{\prime}(\mathrm{T})$ is the spectral synthesis problem which is discussed in $\S 4$ below. However, for open sets the problem has an easy solution which follows from (1.1).

1.3. THEOREM $\left({ }^{4}\right)$. If $\Omega$ is an open subset of $\hat{\Gamma}$ then $E^{\prime}(\Omega)=E(\Omega)$.

It is obvious that if $\mu \in B(\Gamma)$ and $\hat{\mu}(t)=0$ for $t \in \mathrm{T}$ then $\mu \circ \varphi=0$ for $\varphi \in E(\mathrm{~T})$. What (1.3) tells us is the useful fact that if $\hat{\mu}=0$ on a neighborhood of $\Lambda(\varphi)$ then $\mu \circ \varphi=0$.

The next proposition is another spectral synthesis theorem. It is out of place here in the sense that a proof would normally come at a later stage in the exposition.

1.4. ThEOREM( $\left.{ }^{5}\right)$. If $\varphi \in C(\Gamma)$ has spectrum consisting of a single point, $t$, then $\varphi$ is a constant multiple of $e_{t}$.

(3a) The existence of an absolutely continuous measure satisfying the requirements is simply a restatement of the regularity of the group algebra, see Loomis.

(4) Godement.

(5) Among the many extant proofs of this theorem, the one closest to the spirit of the present paper is that given by SEGAL for $\Gamma=R^{1}$ and generalized in REITER [1] to arbitrary groups. 
(1.2) states that if $t \in \Lambda(\varphi)$ then $e_{t}$ is the strict limit of linear combinations of translates of $\varphi$, i.e. functions of the form $\psi(x)=\sum c_{i} \varphi\left(x+y_{i}\right)$ where the sum is finite. (1.3) says that if $\Omega$ is open, $\varphi \in E^{\prime}(\Omega)$ is the strict limit of trigonometric polynomials with spectra in $\Omega$, i.e. finite sums of the form $\psi(x)$ $=\sum c_{i} e\left(t_{i} x\right)$ with $t_{i} \in \Omega$. Using (1.4) above and (5.4) below it is possible to replace strict limits by narrow limits. The precise statements are:

1.2'. TheOREM( $\left.{ }^{6}\right)$. If $\varphi \in C_{u}(\Gamma)$ and $t \in \Lambda(\varphi)$ then $e_{t}$ is the narrow limit of linear combinations' of translates of $\varphi$.

1.3'. Theorem $\left({ }^{7}\right)$. If $\Omega$ is an open subset of $\hat{\Gamma}$ and $\varphi \in E^{\prime}(\Omega)$ then $\varphi$ is the narrow limit of trigonometric polynomials with spectra in $\Omega$.

We shall not have occasion to use these refinements.

At this point we find it convenient to insert an important lemma. Note that if $\varphi, \psi \in C(\Gamma)$ and $a, b$ are constants then it follows immediately from the definition that $\Lambda(a \varphi+b \psi) \subset \Lambda(\varphi) \cup \Lambda(\psi)$. However, the relationship of $\Lambda(\varphi \psi)$ to $\Lambda(\varphi)$ and $\Lambda(\psi)$ seems to lie considerably deeper.

1.5. Lemma. If $\varphi, \psi \in C(\Gamma)$ and $\Lambda(\psi)$ is compact, then $\Lambda(\varphi \psi) \subset \Lambda(\varphi)+\Lambda(\psi)$.

Proof. Let $\mathrm{A}$ be a set containing $\Lambda(\psi)$ in its interior. According to (1.3), $\psi$ is the strict limit of trigonometric polynomials $\sum c_{i} e_{t_{i}}$ where $t_{i} \in \mathrm{A}$. Since $\Lambda\left(\varphi e_{t}\right)=\Lambda(\varphi)+t$, it follows that $\Lambda\left(\varphi \cdot \sum c_{i} e_{t_{i}}\right) \subset \Lambda(\varphi)+A$ and hence that $\Lambda(\varphi \psi) \subset \Lambda(\varphi)+A$. Since $\Lambda(\psi)$ is compact, $\Lambda(\varphi)+\Lambda(\psi)=\bigcap_{A}\{\Lambda(\varphi)+A\}$ where the intersection is taken over all sets $A$ with $\Lambda(\psi) \subset$ Int A. Thus $\Lambda(\varphi \psi) \subset \Lambda(\varphi)$ $+\Lambda(\psi)$.

It is obvious that if $\varphi \in L^{1} \cap C(\Gamma)$, so that its Fourier transform, $\hat{\varphi}$, is unambiguously defined, then $\Lambda(\varphi)$ is the closure of the set of points where $\hat{\varphi}$ is different from zero. This suggests that the spectrum may be definable in general by using an appropriate summability method $\left({ }^{8}\right)$ to deal with the "integral" $\int \bar{e}(t x) \varphi(x) d x$. This is so, and Riemann's method $(R, 2)$ appears to be the most convenient. The method is generalized according to the following construction. Let $\mathrm{H}$ be a measurable subset of $\hat{\Gamma}$ with finite measure. As a matter of general notation we write $\hat{\chi}_{\mathrm{H}}$ for the characteristic function of the set and $\chi_{\mathrm{B}}$ for its Fourier transform; also $\mathrm{H}^{0}$ denotes Int $\mathrm{H}-\mathrm{Int} \mathrm{H}$. In the specific construction here, $\mathrm{H}$ is assumed to be a $J$-set and $h=|\mathrm{H}|$. Define $k_{\mathrm{H}}(x)=h^{-2}\left|\chi_{\mathrm{H}}(x)\right|^{2}$ and $\omega_{\mathrm{H}}(x)=\sup _{t \in \mathrm{H}}|1-e(t x)|$. The basic facts about $k_{\mathrm{H}}$ are summarized below. Note that $\omega_{\mathrm{H}}$ is a continuous invariant pseudometric

(8) This theorem is due to Beurling (unpublished). See Domar, $\$ 4$, for a proof.

( ${ }^{7}$ ) See $\$ 5$, below, where a generalization of this is given.

(8) The early work of Beurling, e.g. BeurLIng [4], used Abel summability and complex variable methods. The advantages of Riemann's method for bounded functions were shown by Pollard. Herz [2] examines rather general summability methods. However, all the foregoing is restricted to the real line. The proper generalization of Riemann's method to groups is due implicitly to REITER [1]. 
on $\Gamma$. (In the case $\Gamma=R^{1}$ one can take the half-open interval $(-h / 2, h / 2)$ for $\mathrm{H}$; then $k_{\mathrm{H}}(x)=(h x / 2)^{-2} \sin ^{2} h x / 2$.)

\subsection{Lemma. $k_{\mathrm{H}}$ has the properties}

(i) $k_{\mathrm{H}} \in C_{0}(\Gamma)$ and $0 \leqq k_{\mathrm{H}}(x) \leqq k_{\mathrm{H}}(0)=1$;

(ii) $k_{\mathrm{H}} \in L^{1}(\Gamma)$ and $\int k_{\mathrm{H}}(x) d x=h^{-1}$;

(iii) $\hat{k}_{\mathrm{H}} \geqq 0$ and vanishes outside $\mathrm{H}^{0}$;

(iv) $\left|k_{\mathrm{H}}(x-y)-k_{\mathrm{H}}\left(x^{\prime}-y\right)\right| \leqq 2 \omega_{\mathrm{H}}\left(x-x^{\prime}\right)$ uniformly in $y$;

(v) $\int\left|k_{\mathrm{H}}(x-y)-k_{\mathrm{H}}\left(x^{\prime}-y\right)\right| d y \leqq 2 h^{-1} \omega_{\mathrm{H}}\left(x-x^{\prime}\right)$.

Proof. (i) and (ii) are easy to prove; so is (iii) when one observes that for a $J$-set $\mathrm{H}, k_{\mathrm{H}}$ is unaltered when $\mathrm{H}$ is replaced by Int $\mathrm{H}$. To prove (iv) and (v) we use $\left|k_{\mathrm{H}}(x-y)-k_{\mathrm{H}}\left(x^{\prime}-y\right)\right| \leqq h^{-2}\left|\chi_{\mathrm{H}}^{2}(x-y)-\chi_{\mathrm{H}}^{2}\left(x^{\prime}-y\right)\right|=h^{-1} \mid \chi_{\mathrm{H}}(x-y)$ $+\chi_{H}\left(x^{\prime}-y\right)\left|\cdot h^{-1} \chi_{H}(x-y)-\chi_{H}\left(x^{\prime}-y\right)\right|$. The first term of the product is the absolute value of the Fourier transform of $\left[e(t x)+e\left(t x^{\prime}\right)\right] \cdot h^{-1} \hat{\chi}_{H}(t)$ so that it is bounded by 2 and its $L^{2}$-norm is $\leqq 2 h^{-1 / 2}$ according to the Plancherel theorem. Similarly, the second term is the absolute value of the Fourier transform of $\left[e(t x)-e\left(t x^{\prime}\right)\right] \cdot h^{-1} \hat{\chi}_{\mathrm{H}}(t)$, so that it is bounded by $\omega_{\mathrm{H}}\left(x-x^{\prime}\right)$ and its $L^{2}$-norm by $h^{-1 / 2} \omega_{\mathrm{H}}\left(x-x^{\prime}\right)$. (iv) follows immediately; (v) via the Schwarz inequality.

If $\mathrm{H}$ is "small," $k_{\mathrm{H}} \varphi$ is "near" $\varphi$ in the sense that $\left|\varphi(x)-k_{\mathrm{H}}(x) \varphi(x)\right|$ $=|\varphi(x)|\left|k_{\mathrm{H}}(0)-k_{\mathrm{H}}(x)\right| \leqq\|\varphi\| \omega_{\mathrm{H}}(x)$. A family $\{\mathrm{H}\}$ of $J$-sets will be called a " $J$-net" if given any nucleus $\mathrm{N}$ in $\hat{\Gamma}$ there is an $\mathrm{H}$ of the family with $\mathrm{H} \subset \mathrm{N}$. For a $J$-net, $k_{\mathrm{H}} \varphi \rightarrow \varphi$ narrowly as $\mathrm{H} \rightarrow 0$. On the other hand, for each $\mathrm{H}$, $k_{\mathrm{H}} \varphi \in L^{1}(\Gamma)$, so that one may form $\Phi_{\mathrm{H}}(t)=\int \bar{e}(t x) k_{\mathrm{H}}(x) \varphi(x) d x$. The most elementary observations about $\Phi_{\mathrm{H}}$ lead to some important results.

1.7. Theorem. Suppose $\varphi \in C(\Gamma)$; then $\Phi_{\mathrm{H}}$ vanishes outside the set $\Lambda(\varphi)+\mathrm{H}^{0}$. Conversly, if $t \in \Gamma$ and for some nucleus $\mathrm{N}, \lim _{\mathrm{H} \rightarrow 0} \int_{t+\mathrm{N}}\left|\Phi_{\mathrm{H}}(s)\right| d s=0$, then $t \notin \Lambda(\varphi)$.

Proof. Let $\mathrm{A}$ be a closed set of positive measure with $\mathrm{A} \subset$ Int $\mathrm{H}$. For the function $k_{\mathrm{A}}$ we have $\Lambda\left(k_{\mathrm{A}}\right) \subset \mathrm{A}-\mathrm{A}$. By (1.5), $\Lambda\left(k_{\mathrm{A}} \varphi\right) \subset \Lambda(\varphi)+\mathrm{A}-\mathrm{A}$. However $k_{\mathrm{A}} \varphi \in L^{1}(\Gamma)$; so one may conclude that $\Phi_{\mathrm{A}}$, the Fourier transform of $k_{\mathrm{A}} \varphi$, vanishes outside $\Lambda(\varphi)+\mathrm{A}-\mathrm{A}$. Now for the $J$-set $\mathrm{H}$ we can find a sequence of A's such that $|\mathrm{H} \cap \tilde{\mathrm{A}}| \rightarrow 0$. Accordingly, $k_{\mathrm{A}} \rightarrow k_{\mathrm{H}}$ in the mean of $L^{1}(\Gamma)$, and therefore $\Phi_{A} \rightarrow \Phi_{H}$ uniformly. $\Phi_{A}$ vanishes outside $\Lambda(\varphi)+H^{0}$ for each $A$; so the first statement is proved. To prove the second assertion, choose an $f \in L^{1}(\Gamma)$ such that $\hat{f}(t) \neq 0$ while $\hat{f}(s)=0$ for $s \notin t+$ N. $f \circ \varphi(x)$ $=\lim _{\mathrm{H} \rightarrow 0} \int f(x-y) k_{\mathrm{H}}(y) \varphi(y) d y=\lim _{\mathrm{H} \rightarrow 0} \int e(s x) \hat{f}(s) \Phi_{\mathrm{H}}(s) d s$ by the Parseval relation. The hypotheses ensure that the limit (which exists!) must be zero. Therefore $t \notin \Lambda(\varphi)$.

For $\varphi \in C(\Gamma),\left\|\Phi_{\mathrm{H}}\right\| \leqq h^{-1}\|\varphi\|$ and $\left\|\Phi_{\mathrm{H}}\right\|_{2} \leqq h^{-1 / 2}\|\varphi\|$. The first inequality cannot be improved, and in the second the best one can do is to sharpen the $h^{-1 / 2}$ to something between $|\mathrm{H}|^{-1 / 2}$ and $\left|\mathrm{H}^{0}\right|^{-1 / 2}$. When $\Lambda(\varphi)$ is compact, $\Phi_{\mathrm{H}} \in L^{1}(\hat{\Gamma})$ and $\left\|\Phi_{\mathrm{H}}\right\|_{1}=O\left(h^{-1 / 2}\right)$ as $\mathrm{H} \rightarrow 0$ since the $L^{2}$-norm dominates the 
$L^{1}$-norm for functions vanishing outside compact sets. At least for the groups considered in classical analysis, the last estimate cannot be improved as will be shown by example. Thus, as $\mathrm{H} \rightarrow 0, \Phi_{\mathrm{H}}$ must in general be rather badly behaved. This is further illustrated by the next trivial theorem.

1.8. Theorem. A necessary and sufficient condition for $\varphi \in C(\Gamma)$ to be a Fourier-Stieltjes transform is that $\left\|\Phi_{\mathrm{H}}\right\|_{1}$ be uniformly bounded for a J-net $\{\mathbf{H}\}$. $\varphi$ is positive definite, i.e. the Fourier-Stieltjes transform of a non-negative measure, if and only if $\Phi_{\mathrm{H}} \geqq 0$ for a J-net $\{\mathrm{H}\}$.

(1.7) helps to give some information about spectral sets. If $\mathrm{T}$ is a closed, $\sigma$-compact subset of $\hat{\Gamma}$ one can find an $\alpha \in B(\hat{\Gamma})$ such that $\Sigma(\alpha)=\mathrm{T}$. Thus $\mathrm{T}$ is the spectrum of some function in $C(\Gamma)$, in fact in $C_{u}(\Gamma)$. It is not true in general that if $\varphi \in C(\Gamma)$ then $\Lambda(\varphi)$ is $\sigma$-compact; a counter-example is given at the end of this section. It is, of course, true if $\hat{\Gamma}$ is $\sigma$-compact. However the same assertion holds if $\Gamma$ is $\sigma$-compact. In this case there is a countable $J$-net $\{H\}$ in $\hat{\Gamma}$. Since $\Phi_{H} \in L^{2}(\hat{\Gamma})$, each $\Phi_{H}$ vanishes outside a $\sigma$-compact set. From (1.7) we can conclude that $\Lambda(\varphi)$ is contained in some $\sigma$-compact subgroup of $\hat{\Gamma}$. When dealing with uniformly continuous functions one does not have to impose any restrictions on the group. This is shown in the next theorem, the proof of which illustrates many important techniques.

1.9. Theorem. Given $\varphi \in C_{u}(\Gamma), \Lambda(\varphi)$ is $\sigma$-compact. Corresponding to each compact set $\mathrm{T} \subset \hat{\mathrm{T}}$, there is a $\varphi_{0} \in E^{\prime}(\mathrm{T})$ which is a best approximation to $\varphi$ in the sense that among all functions $\psi \in E^{\prime}(\mathrm{T})$, the quantity $\|\varphi-\psi\|$ attains its minimum for $\psi=\varphi_{0}$. If $\left\{\mathrm{T}_{m}\right\}$ is an increasing sequence of compact sets which cover $\Lambda(\varphi)$, in the sense that for each $t \in \Lambda(\varphi)$ there exists a nucleus $M$ and an integer $m$ such that $(t+\mathrm{M}) \cap \Lambda(\varphi) \subset \mathrm{T}_{m}$, then $\varphi_{m} \rightarrow \varphi$ uniformly, $\varphi_{m}$ being a best approximation to $\varphi$ corresponding to $\mathrm{T}_{m}$.

Proof. We first demonstrate the existence of best approximations. Given $\varphi$ and T, let $F$ be the subset of $E^{\prime}(\mathrm{T})$ consisting of functions with norm $\leqq 2\|\varphi\|$. Since $\mathrm{T}$ is compact there exists an $f \in L^{1}(\Gamma)$ with $\hat{f}=1$ on a neighborhood of T. As a consequence of (1.3), $\psi=f \circ \psi$ for each $\psi \in E^{\prime}(\mathrm{T})$, and it follows that $F$ is a bounded family of uniformly equicontinuous functions. Thus $F$, which is strictly closed, is strictly compact. A fortiori, $\varphi-F$ is strictly compact. A strictly compact subset of $C(\Gamma)$ has an element of minimum norm; $\varphi_{0}$ is chosen so that $\varphi-\varphi_{0}$ is such an element in $\varphi-F$. Next let $\mathrm{N}$ be a nucleus in $\Gamma$ and set $f_{\mathrm{N}}(x)=|\mathrm{N}|^{-1}$ for $x \in \mathrm{N}, f_{\mathrm{N}}=0$ otherwise. Since $\varphi$ is uniformly continuous, it is uniformly approximable by the functions $f_{\mathrm{N}} \circ \varphi$. The functions with Fourier transforms vanishing outside a compact set are dense in $L^{1}(\Gamma)$; thus the functions $f_{N} \circ \varphi$ are uniformly approximable by functions of the form $f \circ \varphi$ where $\hat{f}$ vanishes outside a compact set. It follows that there exists as sequence $\left\{f_{n}\right\}$ of functions in $L^{1}(\Gamma)$ whose Fourier transforms vanish outside compact sets such that $f_{n} \circ \varphi \rightarrow \varphi$ uniformly. $\Lambda\left(f_{n} \circ \varphi\right) \subset \Lambda\left(f_{n}\right) \cap \Lambda(\varphi)$ 
which is compact; so are each of the sets $\Lambda\left(f_{n} \circ \varphi\right)+\bar{M}$ where $\mathbf{M}$ is a nucleus in $\hat{\Gamma}$. We claim that, for any nucleus $M, \Lambda(\varphi) \subset \bigcup_{n=1}^{\infty}\left\{\Lambda\left(f_{n} \circ \varphi\right)+\bar{M}\right\}$, so that, in particular, $\Lambda(\varphi)$ is $\sigma$-compact. Were this not true there would be a $t \in \Lambda(\varphi)$ with $t \notin \Lambda\left(f_{n} \circ \varphi\right)+\bar{M}$ for any $n$. Choose $g \in L^{1}(\Gamma)$ such that $\hat{g}(t)=1$ and $\hat{g}=0$ outside $t-M$. $\hat{g}$ vanishes on a neighborhood of $\Lambda\left(f_{n} \circ \varphi\right)$ which implies that $g \circ\left(f_{n} \circ \varphi\right)=0$ according to (1.3). Since this holds for each $n, g \circ \varphi$ $=\lim g \circ\left(f_{n} \circ \varphi\right)=0$ contradicting the assumption $t \in \Lambda(\varphi)$. The Heine-Borel theorem may be applied to show that given the family of sets $\left\{\mathrm{T}_{n}\right\}$, for each $n$ there is an $m_{n}$ such that $\Lambda\left(f_{n} \circ \varphi\right) \subset \mathrm{T}_{m_{n}}$. By definition, $\left\|\varphi-\varphi_{m_{n}}\right\|$ $\leqq\left\|\varphi-f_{n} \circ \varphi\right\| . \mathrm{T}_{m}$ being an increasing sequence of sets, $\varphi_{m}$ converges uniformly to $\varphi$.

The above is typical of the type of argument where approximation by functions with compact spectrum is used. It is worthwhile noting a few facts about functions with compact spectrum. We have already employed the device of writing $\varphi \in E^{\prime}(\mathrm{T})$, with T compact, as $\varphi=f \circ \varphi$ where $f \in L^{1}(\Gamma)$ and $\hat{f}=1$ on a neighborhood of T. This shows that $\varphi$ is a very "smooth" function, e.g. $\varphi \in C_{u}(\Gamma)$. Moreover $\Phi_{\mathrm{H}} \in L^{1}(\hat{\Gamma})$ so that $\varphi$ is the limit, in a rather strong sense, of Fourier transforms $\int e(t x) \Phi_{\mathrm{H}}(t) d t$ where the domain of integration is eventually an arbitrarily small neighborhood of $\Lambda(\varphi)$. In the case $\Gamma=R^{1}$, it is implicit in Theorem X of PALEY and Wiener that this implies $\varphi$ is the restriction to real arguments of an entire function of exponential type. There is accordingly an intimate connection between the theory of entire functions of exponential type and the theory of functions with compact spectrum( $\left.{ }^{9}\right)$. This topic is discussed more fully in $\$ 5$, but the reader is invited to keep it in mind as a guide to the interpretation in classical analysis of many of the results of this paper.

The subject of spectral analysis has two further aspects which we have not indicated. One is the problem of gaining information about $\Lambda(\varphi)$ from some basic properties of $\varphi$ without necessarily assuming a complete knowledge of the function itself. For example, it follows from the results of $\$ 2$ that if $\varphi \in C_{0}(\Gamma)$ then $\Lambda(\varphi)$ is perfect (unless $\varphi$ is the null function). In this connection, consider a definition.

Definition. $A$ closed set $\mathrm{T}$ in $\hat{\Gamma}$ is a set of multiplicity if $C_{0}(\Gamma) \cap E^{\prime}(\mathrm{T}) \neq 0$.

This definition is easily seen to be equivalent to the usual one $\left({ }^{10}\right)$. However, it is actually more convenient to use. Also some questions present themselves naturally which might otherwise remain obscure, e.g. if $\mathrm{T}$ is a set of multiplicity is $C_{0}(\Gamma) \cap E(\mathrm{~T}) \neq 0$ ? This subject is a very difficult one; in $\S 3$ we discuss an analogous but simpler situation which may be attacked en-

(9) Thus (1.4) for $\Gamma=R^{1}$ and $t=0$ is equivalent to the statement that an entire function of minimal exponential type which is bounded on a line is a constant. This is the proof of (1.4) given in Beurling [1].

(10) Compare Pyatetskir-Shapiro. It will also be instructive to compare many of the re sults of this section and Theorem (4.5) below, for the case $\Gamma=I^{1}$, with ZYGMUND, Chapter XI. 
tirely successfully by developing potential theory on groups. Another example is the study of the case where $\varphi$ is uniformly almost periodic. Here $\Lambda(\varphi)$ is the closure of the spectrum in the sense of Bohr; this is related to the material of $\$ 2$, but one can introduce a more profound consideration. For the given group $\Gamma$, let $\rho: \Gamma \rightarrow P$ be the natural representation into its Bohr compactification: $\rho$ and $\mathrm{P}$ are characterized by saying that $\hat{\mathrm{P}}$ is a discrete group and $\hat{\rho}: \hat{\mathrm{P}} \rightarrow \hat{\Gamma}$ is one-to-one and onto. To say that $\varphi \in C(\Gamma)$ is uniformly almost periodic is to say that $\varphi=\hat{\rho} \psi$ where $\psi \in C(\mathrm{P})$. The problem is then to study the effect on the spectrum of the map $\hat{\rho}: C(\mathrm{P}) \rightarrow C(\Gamma)$. The question no longer requires a consideration of almost periodic functions but arises whenever one has a representation of one group in another. That is the topic which concerns us in $\$ 5$.

The second aspect of spectral analysis to be mentioned is that of altering the definition in such a way that the spectrum is defined for other classes of functions and is consistent with the original definition for bounded continuous functions. There are three basic procedures which have been used. First, one can use the original definition with a more restrictive set of measures allowed for consideration. For instance, let $A(\Gamma)$ be a subalgebra of $L^{1}(\Gamma)$ which is a regular, semi-simple Banach algebra with involution having the same maximal ideal space as $L^{1}(\Gamma)$. We then admit only measures $\mu \in B(\Gamma)$ of the form $\mu(d x)=f(x) d x$ where $f \in A(\Gamma)$. This procedure is discussed by DomaR( $\left.{ }^{11}\right)$.

A second procedure involves taking a topological linear space of functions, in which the translation operations are continuous and considering the closed, translation-invariant subspaces. To a given function, one associates the smallest closed translation-invariant subspace containing it. The spectrum is defined by assigning to each such subspace, a point set, e.g., given a closed translation-invariant subspace $E$, we might assign to $E$ the subset $\bigcup_{\phi \in E} \Lambda(\varphi)$ where the functions $\varphi$ are restricted to belong to some special class of bounded continuous functions, cf. (1.2). This device is used in §3. Such a definition of spectrum may not be consistent with the original definition for bounded continuous functions in the space, and the proof of consistency in a given instance may be quite difficult. The third method of extending the notion of spectrum, is to use summability methods with the definition of spectrum being analogous to the statement of (1.7), cf. BEURLING [4].

Some examples will now be given. If the group $\Gamma$ is compact, one may choose the $J$-set $\mathrm{H}$ to be $\{0\} . \Phi_{\mathrm{H}}$ is then simply the ordinary Fourier transform of $\varphi$ and everything is trivial. Therefore we shall consider only noncompact groups, $\Gamma$.

ExAmple. $\Gamma=I^{1}$ with the Haar measure being the counting measure. The space $\hat{\Gamma}$ may be identified with the interval $(-\pi, \pi]$; the dual Haar measure is $(2 \pi)^{-1}$ times Lebesgue measure and the characters are chosen as $e(t x)$ $=\exp (i t x)$. For $h \leqq 1 / 2$, let $\mathrm{H}=(-\pi h, \pi h)$; then $k_{\mathrm{H}}(x)=(\pi h x)^{-2} \sin ^{2} \pi h x$.

(11) See also BeurLING [3] where the idea originated. 
Given $\varphi \in C(\Gamma)$, i.e. a bounded sequence, set $F(t)=1 / 2 \varphi(0) t^{2}$ $-\sum_{x \neq 0} \exp (-i t x) x^{-2} \varphi(x)$. Then

$$
\Phi_{\mathrm{H}}(t)=1 / 2(\pi h)^{-2}\{F(t+\pi h)-2 F(t)+F(t+\pi h)\} .
$$

Thus the second derivative of $F$ is zero at every point of $\tilde{\Lambda}(\varphi)$, the complement of the spectrum, and that is the largest open set in which the statement holds. One can use Abel summability instead of $(R, 2)$ summability to take advantage of known results in function theory. One sets $\Phi(r ; t)$ $=\sum \varphi(x) r^{|x|} \exp (-i t x), 0<r<1 ; \lim _{r \rightarrow 1} \Phi(r ; t)=0$ if $t \notin \Lambda(\varphi)$. It follows from the theory of analytic functions that if $\varphi(x)=0$ for $x<0$ then $\Lambda(\varphi)=\hat{\Gamma}$ or $\phi$.

To show that the estimate $\left\|\Phi_{\mathrm{H}}\right\|_{1}=O\left(h^{-1 / 2}\right)$ cannot be improved, introduce a parameter $\xi, 0 \leqq \xi \leqq 1$ and set $\varphi(x)=0$ for $x \leqq 0, \varphi(x)=\varphi(x, \xi)$, the $x$ th Rademacher function when $x=1,2, \cdots$. It is known $\left({ }^{12}\right)$ that there exists a constant $B$ such that if $f \in L^{1}(I)$, then

$$
\sum_{x=1}^{\infty}|f(x)|^{2} \leqq B\left\{\int_{0}^{1}\left|\sum_{x=1}^{\infty} f(x) \phi(x, \xi)\right| d \xi\right\}^{2} .
$$

Given $t \in \hat{\Gamma}$, set $f=\bar{e}_{t} k_{\mathrm{H}}$. From the previous remark it follows that there is a universal constant $A>0$ such that $A^{2} h^{-1} \leqq\left\{\int_{0}^{1} \sum_{x} \bar{e}(t x) k_{\mathrm{H}}(x) \varphi(x, \xi) \mid d \xi\right\}^{2}$ $=\left\{\int_{0}^{1}\left|\Phi_{\mathrm{H}}(t, \xi)\right| d \xi\right\}^{2}$. Thus $(2 \pi)^{-1} \int_{-\pi}^{\pi} \int_{0}^{1}\left|\Phi_{\mathrm{H}}(t, \xi)\right| d \xi d t \geqq A h^{-1 / 2}$. Interchanging the order of integration, we see that for any fixed countable $J$-net $\{\mathbf{H}\}$, there is a $\xi$-set of positive measure in which $\lim \sup _{\mathrm{H} \rightarrow 0} h^{1 / 2}\left\|\Phi_{\mathrm{H}}(\cdot, \xi)\right\|_{1}>0$.

EXAmple. $\Gamma=R^{r}$ with Haar measure being $(2 \pi)^{-r}$ times Lebesgue measure. Taking $e(t x)=\exp \left\{i\left(t_{1} x_{1}+\cdots+t_{r} x_{r}\right)\right\}$, one finds that $\widehat{\Gamma}=R^{r}$ with ordinary Lebesgue measure as the dual Haar measure. Assuming that $\int_{|x|>1}|x|^{-2}|\varphi(x)| d x$ $<\infty$, one can form the 2 -transform

$$
F(t)=\int_{|x| \leq 1}\{\bar{e}(t x)-1+i t \cdot x\}|x|^{-2} \varphi(x) d x+\int_{|x|>1} \bar{e}(t x)|x|^{-2} \varphi(x) d x .
$$

Now suppose $\varphi \in C(\Gamma)$. Let $F_{\mathrm{H}}$ be the 2-transform of $k_{\mathrm{H}} \varphi$ and set $D=-\partial^{2} / \partial t_{1}^{2}$ $+\cdots-\partial^{2} / \partial t_{r}^{2}$. If $\{\mathbf{H}\}$ is a $J$-net, $F_{\mathbf{H}} \rightarrow F$ uniformly as $\mathbf{H} \rightarrow 0$ and $D \circ F_{\mathbf{H}}$ $=\Phi_{\mathrm{H}}$. It follows that $F$ is harmonic, i.e. $D \circ F=0$, in $\tilde{\Lambda}(\varphi)$.

EXAMPLE. Suppose $\Gamma$ is the union of an increasing collection of compact open subgroups $\{\mathrm{K}\}$. One can take a $J$-net to be $\left\{\mathrm{H}_{\mathbf{K}}\right\}$ where $\mathrm{H}_{\mathbf{K}}=\mathrm{K}^{\perp}$, the annihilator subgroup of $\mathrm{K}$ in $\hat{\Gamma}$, i.e. $\mathrm{H}_{\mathrm{K}}=\{t \mid e(t x)=1$ for $x \in \mathrm{K}\} . k_{\mathrm{H}_{\mathrm{K}}}$ is the function which is 1 on $\mathrm{K}$ and 0 elsewhere. The group $\Gamma$ is of rank 0 , but the spectral theory is nontrivial if $\Gamma$ is not compact; such a group is a good example to bear in mind.

ExAmple. Let $\Gamma_{1}$ be an uncountable discrete group and $\Gamma_{2}$ a group isomorphic to $\hat{\Gamma}_{1}$. Set $\Gamma=\Gamma_{1} \oplus \Gamma_{2}$; then $\hat{\Gamma}=\hat{\Gamma}_{1} \oplus \hat{\Gamma}_{2}$ where $\hat{\Gamma}_{2}$ is isomorphic to $\Gamma_{1}$; let $x \rightarrow t_{x}$ with $x \in \Gamma_{1}, t_{x} \in \hat{\Gamma}_{2}$ be an explicit isomorphism. For $x \in \Gamma_{1}, y \in \Gamma_{2}$

(12) KACZMARZ and Steinhaus [1, \$714]. 
define $\varphi(x, y)=e\left(t_{x} y\right)$. This function is continuous; we shall show that $\Lambda(\varphi)$ $=\hat{\Gamma}$, which is not $\sigma$-compact. To do this, choose a fixed $z \in \Gamma_{1}$ and define $f \in L^{1}(\Gamma)$ by $f(x, y)=0$ for $x \neq 0$ and $f(0, y)=e\left(t_{z} y\right)$. Set $g=f \circ \varphi$. One has $g(x, y)=\int_{\Gamma_{2}} d v \int_{\Gamma_{1}} d u \varphi(x-u, y-v) f(u, v)=\int_{\Gamma_{2}} e\left[t_{x}(y-v)\right] e\left(t_{z} v\right) d v$. Since $\Gamma_{2}$ is compact we obtain $g(x, y)=0$ for $x \neq z, g(z, y)=e\left(t_{z} y\right)$. It is easy to check that $g$ is the Fourier transform of $\hat{g} \in L^{1}(\hat{\Gamma})$ where $\hat{g}(s, t)=0$ for $t \neq t_{z}$ and $\hat{g}\left(s, t_{z}\right)$ $=\bar{e}(s z)$. Thus $\Lambda(g)=\hat{\Gamma}_{1}+t_{z}$, but $\Lambda(g) \subset \Lambda(\varphi)$ and $z \in \Gamma_{1}$ was entirely arbitrary. Thus the assertion $\Lambda(\varphi)=\hat{\Gamma}$ is proved.

2. The point spectrum. We now turn our attention to defining and analyzing the point spectrum of a uniformly continuous function. In case $\phi \in C_{u}(\Gamma)$ has the form $\phi(x)=\int e(t x) \alpha(d t)$ where $\alpha \in B(\hat{\Gamma})$, then $\Lambda_{p}(\phi)=\Sigma_{p}(\alpha)$ corresponds to the Bohr spectrum of the almost periodic part of $\phi$. More generally, if $\phi$ is any weakly almost periodic function $\left({ }^{13}\right)$ on $\Gamma$ there is an obvious choice for $\Lambda_{p}(\phi)$. However, in the general case it is not entirely clear what the point spectrum ought to be; we have chosen a maximal definition, that is the largest set which could reasonably be called a "point spectrum." This is accomplished by taking into account all possible extensions of the notion of "almost periodic mean."

Definition. A linear functional $\mathfrak{T}$ defined on $C_{u}(\Gamma)$ will be called an "invariant mean" $\left.{ }^{14}\right)$ if it satisfies

(M1) $\operatorname{Tr}(1)=1$,

(M2) $|\mathfrak{T}(\phi)| \leqq\|\phi\|$,

(M3) $\mathfrak{N}_{x}\{\phi(x+y)\}=\mathfrak{N}_{x}\{\phi(x)\}$ for each $y \in \Gamma$.

To study invariant means more closely one introduces the set $A_{\phi}$, the smallest, translation-invariant convex subset of $\dot{C}_{u}(\Gamma)$ containing $\phi$. Define $M(\phi)=\inf \|\psi\|$ for $\psi \in A_{\phi}$. The properties of $M$ are summarized below.

2.1. TheOREM. $M$ is a pseudo-norm on $C_{u}(\Gamma)$ with the properties: $M(1)=1$, $M(\phi) \leqq\|\phi\|$, and $M_{x}\{\phi(x+y)\}=M_{x}\{\phi(x)\}$ for each $y \in \Gamma$. A linear functional $\mathfrak{N C}$ defined on $C_{u}(\Gamma)$ is an invariant mean if and only if $\mathfrak{T C}(1)=1$ and $|\mathfrak{T H}(\phi)|$ $\leqq M(\phi)$. For a given $\phi \in C_{u}(\Gamma), M(\phi)=\max |\mathfrak{T}(\phi)|$ where the maximum is taken over all invariant means.

Proof. The first two statements are due to Banach( $\left.{ }^{15}\right)$. To prove the last one we take a closer look at $M(\phi)$. For any function $\psi$, let $\langle\psi\rangle$ be its convex cover, i.e. the smallest closed convex set in the complex plane containing the points $\psi(x), x \in \Gamma$. Set $\left\langle A_{\phi}\right\rangle=\bigcap\langle\psi\rangle, \psi \in A_{\phi}$. By the Hahn-Banach theorem, there exists a linear functional $L$ satisfying (M1) and (M2) with $L(\psi)=z$ if and only if $z \in\langle\psi\rangle$. Hence $\left\langle A_{\phi}\right\rangle$ is exactly the set of complex numbers of the form $z=\mathfrak{M}(\phi)$ for some invariant mean $\mathfrak{T}$. We wish to show that $M(\phi)$

(18) See EBERLEIN.

(14) These are essentially the generalized limits of BANACH, Chapter II, \$3.

(15) Loc. Cit. The third statement is proved here since the modification required to treat complex-valued functions is not entirely obvious. 
$=\max |z|, z \in\left\langle A_{\phi}\right\rangle$. To do this observe that any $\psi \in A_{\phi}$ has the form $\psi(x)$ $=\sum a_{i} \phi\left(x+y_{i}\right)$ where $a_{i} \geqq 0, \sum a_{i}=1$ and $y_{i} \in \Gamma$. If $\psi^{\prime}(x)=\sum b_{j} \phi\left(x+y_{j}\right) \in A_{\phi}$ then $\psi^{\prime \prime}(x)=\sum a_{i} b_{j} \phi\left(x+y_{i}+y_{j}\right) \in A_{\phi}$. Moreover $\left\langle\psi^{\prime \prime}\right\rangle \subset\langle\psi\rangle \cap\left\langle\psi^{\prime}\right\rangle$. Now if $\psi \in A_{\phi},\langle\psi\rangle$ meets the ring $M(\phi) \leqq|z| \leqq\|\phi\|$. Thus the sets $\langle\psi\rangle, \psi \in A_{\phi}$, and the ring have the finite intersection property. Consequently they have a point in common, $z$, where $z \in\left\langle A_{\phi}\right\rangle$ and $|z| \geqq M(\phi)$.

This result enables us to define the point spectrum $\left({ }^{16}\right)$.

Definition. For $\phi \in C_{u}(\Gamma), \Lambda_{p}(\phi)=\left\{t \in \hat{\Gamma} \mid M\left(\bar{e}_{t} \phi\right) \neq 0\right\}$. The basic facts about the point spectrum may be summed up in one theorem.

2.2. Theorem. If $\phi \in C_{u}(\Gamma)$ then $\Lambda_{p}(\phi) \subset \Lambda(\phi) . \Lambda_{p}(\phi)=\bigcup \Lambda_{p}(\rho)$ where $\rho(y)$ $=\mathfrak{M}_{x}\{\phi(x+y) \Phi(x)\}$, the union being taken over all invariant means. Moreover, for $\mu \in B(\Gamma), \Lambda_{p}(\mu \circ \phi)=\tilde{Z}(\mu) \cap \Lambda_{p}(\phi)$. If $\Lambda(\phi) \subset Z(\mu)$ then $M\{|\mu \circ \phi|\}=0$.

Proof. The essential point is the familiar device of introducing $\rho(y)$ $=\mathfrak{M}_{x}\{\phi(x+y) \phi(x)\}$, where $\mathfrak{M}$ is some invariant mean. This is the only place where the uniform continuity of $\phi$ is used, to assert that $\rho$ is a continuous positive definite function. Thus $\rho(y)=\int e(t y) \sigma(d t)$ where $\sigma$ is a finite non-negative Radon measure on $\hat{\Gamma}$. Indeed

$\mathfrak{M}_{x}\{\mu \circ \phi(x+y) \bar{\mu} \circ \bar{\phi}(x)\}=\int \rho\left(y-x+x^{\prime}\right) \mu(d x) \bar{\mu}\left(d x^{\prime}\right)=\int e(t y)|\hat{\mu}(t)|^{2} \sigma(d t)$.

The entire theorem rests upon the assertion $\Lambda_{p}(\phi)=\cap \Lambda_{p}(\rho)$ for $\Lambda_{p}(\rho)$ $=\Sigma_{p}(\sigma) \subset \Sigma(\sigma)=\Lambda(\rho) \subset \Lambda(\phi)$ and the statements about $\mu$ follow from the equations in the sentence above. What we have to show is that $M\left(\bar{e}_{t} \rho\right)=0$ is equivalent to $\mathbb{M}\left(\bar{e}_{t} \phi\right)=0$. Take $t=0$ for simplicity. If $M(\rho)=0$ then $\left\langle A_{\rho}\right\rangle$ $=\{0\}$ so that $0 \in \bar{A}_{\rho}$, the closure being taken in the uniform topology. Thus given $\epsilon>0$, there is a finite collection of pairs $\left(a_{i}, y_{i}\right) ; a_{i} \geqq 0, \sum a_{i}=1, y_{i} \in \Gamma$; such that $\left|\sum a_{i} \Re_{x}\left\{\phi\left(x+y_{i}\right) \phi(x-y)\right\}\right|<\epsilon^{2}$ for each $y \in \Gamma$. Therefore $\left|\sum a_{i} \Re_{x}\left\{\phi\left(x+y_{i}\right) \bar{\phi}\left(x+y_{j}\right)\right\}\right|<\epsilon^{2}$. Multiplying by $a_{j}$ and summing over $j$ one has, $\mathfrak{M}_{x}\left\{\left|\sum a_{i} \phi\left(x+y_{i}\right)\right|^{2}\right\}<\epsilon^{2}$. Hence $|\mathfrak{T}(\phi)|=\left|\mathfrak{M}_{x}\left\{\sum a_{i} \phi\left(x+y_{i}\right)\right\}\right|<\epsilon$ by the Schwarz inequality. This argument demonstrates that $M\left(\bar{e}_{t} \rho\right)=0$ implies $M\left(\bar{e}_{t} \phi\right)=0$, and so if $M\left(\bar{e}_{t} \rho\right)=0$ for each $\mathfrak{T}, M\left(\bar{e}_{t} \phi\right)=0$, or $\Lambda_{p}(\phi) \subset \cup \Lambda_{p}(\rho)$. In the converse direction, if $M(\phi)=0$ we can find as above a convex combination of translates with $\left|\sum a_{i} \phi\left(x+y_{i}\right)\right|<\epsilon$ for each $x \in \Gamma$. Thus $\left|\sum a_{i} \phi\left(x+y+y_{i}\right)\right|$ $<\epsilon$ so $\left|\mathfrak{T}_{x}\left\{\sum a_{i} \phi\left(x+y_{i}+y\right) \phi(x)\right\}\right|<\epsilon\|\phi\|$. This says $M(\rho)<\epsilon\|\phi\|$ for arbitrary $\epsilon>0$. Reasoning as before one sees that $\Lambda_{p}(\rho) \subset \Lambda_{p}(\phi)$ for each $\mathfrak{T}$.

There is no difficulty in extending the notion of point spectrum to $L^{\infty}(\Gamma)$. If $\phi \in L^{\infty}(\Gamma)$ and $f \in L^{1}(\Gamma)$ then $f \circ \phi \in C_{u}(\Gamma)$. We define $\Lambda_{p}(\phi)=\cup \Lambda_{p}(f \circ \phi)$ where the $f^{\prime}$ 's run over any collection of functions in $L^{1}(\Gamma)$ whose Fourier transforms have no common zeros. The previous theorem guarantees the consistency of this definition.

(10) The notion is implicit in REITER [1]. 
A valuable elementary result which facilitates the computation of point spectra is the following.

2.3. LEMMA $\left({ }^{17}\right)$. Let $\{\pi\}$ be a directed set of regular probability distributions on $\Gamma$ with the property that $\left(^{*}\right) \int|\pi(d x)-\pi(d x-y)| \rightarrow 0$ for each $y \in \Gamma$. Suppose $\phi \in C_{u}(\Gamma)$; then a necessary and sufficient condition that $M(\phi)=0$ is $\int \phi(x+y) \pi(d x)$ $\rightarrow 0$ uniformly in $y$.

Proof. The sufficiency is trivial and does not make use of $\left(^{*}\right)$. Turning to the necessity, one observes that if $M(\phi)=0$ then given $\epsilon>0$ there is a finite collection of pairs $\left(a_{i}, y_{i}\right) ; a_{i} \geqq 0, \sum a_{i}=1, y_{i} \in \Gamma$; such that $\left|\sum a_{i} \phi\left(x+y_{i}\right)\right|<\epsilon$ for each $x$. Thus

$$
\begin{aligned}
\mid \int \phi(x & +y) \pi(d x) \mid \\
& =\mid \sum a_{i} \int \phi(x+y) \pi\left(d x-y_{i}\right)+\sum a_{i} \int \phi(x+y)\left[\pi(d x)-\pi\left(d x-y_{i}\right)\right] \\
& =\left|\int \sum a_{i} \phi\left(x+y+y_{i}\right) \pi(d x)+\sum a_{i} \int \phi(x+y)\left[\pi(d x)-\pi\left(d x-y_{i}\right)\right]\right| \\
& <\epsilon+\|\phi\| \sum a_{i} \int\left|\pi(d x)-\pi\left(d x-y_{i}\right)\right| .
\end{aligned}
$$

The second term approaches zero according to $\left({ }^{*}\right)$.

Lemma (1.6) guarantees that the condition $\left({ }^{*}\right)$ of $(2.3)$ is fulfilled by the distributions with frequencies $l_{\mathrm{H}}=h k_{\mathrm{H}}$ where $\{\mathrm{H}\}$ is a $J$-net. Thus, a necessary and sufficient condition that $t \notin \Lambda_{p}(\phi)$ is that $\int \bar{e}(t x) l_{\mathrm{H}}(x-y) \phi(x) d x \rightarrow 0$ uniformly in $y$ as $\mathrm{H} \rightarrow 0$. The uniformity restriction appears to be a very severe one as will be seen in the examples at the end of the section. A more modest point spectrum would be obtained by dropping the uniformity; of course in this case the results will depend on the special choice of the $J$-net $\{H\}$. Nevertheless one may envisage a fixed $J$-net $\{\mathrm{H}\}$ and consider the functional $R(\chi)=\lim _{\mathbf{H} \rightarrow 0} \int l_{\mathbf{H}}(x) \chi(x) d x$ for all $\chi \in L^{\infty}(\Gamma)$ for which the limit exists. This is the analogue of Riemann's method $R_{2}^{\prime}$. On its domain of definition in $C_{u}(\Gamma), R$ is an invariant mean. It is natural to consider those $\phi$ 's in $C_{u}(\Gamma)$ for which $\rho(y)=\mathcal{R}_{x}\{\phi(x+y) \phi(x)\}$ exists. Let us set $\Omega(\phi)=\Lambda(\rho), \Omega_{p}(\phi)=\Lambda_{p}(\rho)$. $\Omega(\phi)$ is what we should call the "Wiener spectrum" $\left({ }^{18}\right)$ of $\phi$ and $\Omega_{p}(\phi)$ the "Wiener point spectrum." In case $\Gamma=R^{1}$ and $\mathrm{H}=(-h / 2, h / 2), \Omega(\phi)$ is exactly the Wiener spectrum. As may be seen from (2.2) and its proof, $\Omega(\phi) \subset \Lambda(\phi)$, $\Omega_{p}(\phi) \subset \Lambda_{p}(\phi)$. The first inclusion is usually proper; in fact if $\phi \in C_{0}(\Gamma)$,

(17) LORENTz, Theorem 1.

(18) See WIENER, Chapter IV. Note that Wiener's point spectrum always includes Bohr's. Wiener used the word "spectrum" to denote the energy distribution $\sigma$ above rather than its support. 
$\Omega(\phi)=\phi$. The second inclusion may be proper also, e.g. $\Omega_{p}(\phi)$ is always countable while $\Lambda_{p}(\phi)$ need not be. Indeed, it appears that unless $\phi$ is weakly almost periodic, in which case $\Omega_{p}(\phi)=\Lambda_{p}(\phi)$ and $\Omega(\phi)=\bar{\Lambda}_{p}(\phi)$, the two notions of point spectrum are not likely to agree. If $\phi$ is actually uniformly almost periodic then $\Omega(\phi)=\Lambda(\phi)$.

There is one key feature, however, which all definitions of spectrum have in common. This concerns isolated spectral points.

2.4. Theorem. Suppose $\varphi \in C_{u}(\Gamma)$ and $t$ is an isolated point of $\Lambda(\varphi)$. Then for all invariant means $\mathfrak{N}, \mathfrak{N}\left(\bar{e}_{t} \varphi\right)$ has the same nonzero value.

Proof. Assume for simplicity that $t=0$. Since 0 is isolated there is an $f \in L^{1}(\Gamma)$ with $\hat{f}(0)=1$ and $\hat{f}=0$ on a neighborhood of the rest of $\Lambda(\varphi) . \Lambda(f \circ \varphi)$ $=\{0\} ;$ so $f \circ \varphi$ is a nonzero constant. For any invariant mean, $\mathfrak{T}(f \circ \varphi)$ $=\hat{f}(0) \mathscr{T}(\varphi)$ which proves the assertion. Two consequences of this theorem are worth mentioning. One is that a set of multiplicity must have a perfect subset. The other is that if $\varphi \in C(\Gamma)$ cannot be synthesized from its spectrum then the boundary of $\Lambda(\varphi)$ has a perfect subset; in this we use the last assertion of (2.2).

Rather than continue in any generality let us specialize to $\Gamma=R^{1}$, $\mathrm{H}=(-h / 2, h / 2)$. Then if $\chi \in L^{\infty}(\Gamma)$ and $R(\chi)$ exists, the classical version of Wiener's Tauberian Theorem yields

$$
\Re(\chi)=\lim _{L \rightarrow \infty}(2 L)^{-1} \int_{-L}^{L} \chi(x) d x=\lim _{\epsilon \rightarrow 0} \int_{-\infty}^{\infty} \exp (-\epsilon|x|) \chi(x) d x .
$$

The Cesàro and Abel methods are usually more convenient in computations than is Riemann's.

EXAMPLE. Let $\phi(x)=\sin x$ for $|x| \leqq \pi / 2, \phi(x)=\operatorname{sgn} x$ for $|x|>\pi / 2$. $\Lambda_{p}(\phi)=\{0\}$, in fact $\rho(y)=\Re_{x}\{\phi(x+y) \phi(x)\} \equiv 1$, but $R(\phi)=0$. $\hat{\phi}(t)$ $=\lim _{\mathrm{H} \rightarrow 0} \Phi_{\mathrm{H}}(t)=2 i t^{-1}\left(t^{2}-1\right)^{-1} \cos \pi t / 2$ for $t \neq 0, \hat{\phi}(0)=0$. Thus the formal Fourier transform is singular at the point spectrum even though it exists there.

EXAmple. Take $\phi \in C_{u}$ with $0 \leqq \phi \leqq 1$ such that there are arbitrarily long intervals where $\phi(x) \equiv 1$. Then $M(\phi)=1$; however one can make the intervals where $\phi(x)>0$ so rare that $R\left\{|\phi|^{2}\right\}=0$. Thus $0 \in \Lambda_{p}(\phi)$ while $\Omega(\phi)=\varnothing$.

EXAMPLE. $\phi(x)=\exp \left(i x^{2}\right)$. This rapidly oscillating function is not uniformly continuous so that although $\rho(y)$ exists everywhere it does not furnish much information. Let $f(x)=\exp \left(-x^{2}\right) . \hat{f} \neq 0$ so $\Lambda_{p}(\phi)=\Lambda_{p}(f \circ \phi)$. A simple direct calculation shows that $f \circ \phi \in C_{0}$. Thus $\Lambda_{p}(\phi)=\varnothing$.

EXAMPLE. $\phi(x)=\exp \{i x \sin \log \log x\}$ for $x>e, \phi(x)=0$ for $x \leqq e$. According to (2.3), $t \notin \Lambda_{p}(\phi)$ if and only if $L^{-1} \int_{\nu}^{y+L} \exp (-i t x) \phi(x) d x \rightarrow 0$ uniformly in $y$ as $L \rightarrow \infty$. If $-1 \leqq t \leqq 1$ and $L$ is given, let $\epsilon$ be a small positive number. Choose $y>\exp \left(\epsilon^{-1} L\right)$ such that $\sin \log \log y=t$. Then 


$$
L^{-1} \int_{y}^{y+L} \exp \{i x(\sin \log \log x-t)\} d x=1-O(\epsilon) .
$$

Thus the interval $[-1,1]$ belongs to $\Lambda_{p}(\varphi)$. (In fact equality holds.) From this it is obvious that one can find bounded uniformly continuous functions whose point spectrum is the whole real line.

Note. The phenomenon that the point spectrum can have positive measure when $\Gamma=R^{1}$ is due to the requirement that the mean-value limits exist uniformly. It has been pointed out to me that if $\varphi \in L^{\infty}\left(R^{1}\right)$, then

$$
\lim _{L \rightarrow \infty}(2 L)^{-p} \int_{-L}^{L} \exp (-i t x) \varphi(x) d x=0
$$

for almost all $t$ whenever $p>1 / 2$. The result as stated requires the Rademacher-Menchov argument but elementary reasoning suffices for $p>2 / 3$. The latter argument is applicable to the obvious analogue in any noncompact, $\sigma$-compact group. The Riemann kernel is not quite as well behaved as Cesàro's, but one can prove

2.5. THEOREM. Let $\Gamma$ be a noncompact, $\sigma$-compact group and $\{\mathrm{H}\}$ a decreasing $J$-net in $\Gamma$. Then if $\varphi \in L^{\infty}(\Gamma), h^{p} \Phi_{\mathrm{H}}(t) \rightarrow 0$ almost everywhere as $H \rightarrow 0$ whenever $p>5 / 6$.

The essential point of the proof is that if $H^{\prime} \subset H$ then for $p \leqq 1$, $\left|h^{p} \Phi_{\mathrm{H}}(t)-h^{p} \Phi_{\mathrm{H}^{\prime}}(t)\right| \leqq\|\varphi\| h^{p-1} X$ where $X=\left\{\left(h / h^{\prime}\right)^{2-p}-1+\left(2 h / h^{\prime}-2\right)^{1 / 2}\right\}$. This estimate allows us to replace the $J$-net $\{H\}$ by a judiciously chosen subnet $\left\{\mathrm{H}_{n}\right\}$ so that $\lim _{n \rightarrow \infty} h_{n}^{p} \Phi_{\mathrm{H}_{n}}(t)=0$ implies $\lim _{\rightarrow \mathrm{H} 0} h^{p} \Phi_{\mathrm{H}}(t)=0$ while $\sum_{n}\left\|h_{n}^{p} \Phi_{\mathrm{H}_{n}}\right\|_{2}^{2}<\infty$.

3. Potential theory and spectral analysis $\left({ }^{19}\right)$. To avoid triviality, in this section we assume that $\Gamma$ is noncompact. In a certain sense, if $\varphi \in C(\Gamma)$ is "small at $\infty$ " then $\Lambda(\varphi)$ must be a "big" subset of $\hat{\Gamma}$. Various interpretations of this statement give rise to interesting problems. For example, if by "small" we mean $\varphi \in C_{0}(\Gamma)$ the interpretation of "big" is "set of multiplicity." The characterization of sets of multiplicity is at the moment too difficult. In the program at hand, the size of a set is a sort of Hausdorff dimension. To be precise, the statement " $\varphi$ is small at $\infty$ " will be cast in the form $\int|\varphi(x)|{ }^{2} q(x) d x$ $<\infty$ where $q$ is an appropriate function; the conclusion will be that $\Lambda(\varphi)$ has positive capacity in a certain potential theory.

The potential theories on $\hat{\Gamma}$ will be worked out in terms of operations having many of the essential characteristics of minus the second derivative.

(19) The material of this section is closely related to but independent of some of the unpublished work of Beurling and Deny on "Dirichlet spaces." The original motivation for what is here was an attempt to extend some of the results of BEURLING [2]. For the case of Riesz potentials on Euclidean space the main results were already obtained in BEURLING $[4 ; 5]$ and DENY. I have previously announced some of the theorems in SyMposium. 
Definition. $A$ linear operation $D$ with domain and range in $C(\hat{\Gamma})$ will be called a "generalized Laplacian" if

(D1) $D$ is closed in the strict topology of $C(\hat{\Gamma})$,

(D2) the domain of $D$ includes all the continuous characters,

(D3) $D$ commutes with translations,

(D4) $D$ is real and symmetric, i.e. $(D \circ \theta)^{-}=D \circ \bar{\theta}$ and $(D \circ \theta)^{*}=D \circ \theta^{*}$,

(D5) if $\theta$ belongs to the domain of $D$ and $\theta(0) \leqq \theta(t)$ for each $t \in \Gamma$ then $D \circ \theta(0) \leqq 0\left({ }^{20}\right)$.

The generalized Laplacians are associated with two other classes of entities which we now proceed to define.

Definition. A function $\nu$ defined on $\Gamma$ is "negative definite" ${ }^{21}$ if

(N1) $\nu$ is continuous, $\nu(x) \geqq \nu(0)=0, \nu(-x)=\nu(x)$;

(N2) for each finite collection of pairs $\left(c_{i}, x_{i}\right)$ where $c_{i}$ is a real number, $x_{i} \in \Gamma$, and $\sum c_{i}=0$ one has

$$
\sum c_{i} c_{j} \nu\left(x_{i}-x_{j}\right) \leqq 0 .
$$

Definition. A family of measures $\{\pi(\xi)\} \in B(\Gamma)$ defined for $\xi>0$ is a "symmetric probability semi-group" if

(P1) for each Borel set $\mathrm{B}, \pi(\xi ; \mathrm{B}) \geqq 0$ and $\pi(\xi ; \hat{\Gamma})=1$,

(P2) $\pi(\xi) \circ \pi(\eta)=\pi(\xi+\eta)$,

(P3) for each nucleus $\mathrm{N}, \lim _{\xi \rightarrow 0} \pi(\xi ; \mathrm{N})=1$,

(P4) for each Borel set $\mathrm{B}, \pi(\xi ;-\mathrm{B})=\pi(\xi ; \mathrm{B})$.

With these definitions we can state the main theorem connecting various concepts.

3.1. TheOREM( $\left.{ }^{22}\right)$. The families of generalized Laplacians, negative definite functions, and symmetric probability semi-groups correspond uniquely in triples $\{D, \nu, \pi(\xi)\}$ :

(i) $\nu(x)=D \circ e_{x}(0)$ where $e_{x}(t)=e(t x)$,

(ii) $\int e(t x) d \pi(\xi ; t)=\exp \{-\xi \nu(x)\}$,

(iii) $D \circ \theta=\lim _{\xi \rightarrow 0} \xi^{-1}[\theta-\pi(\xi) \circ \theta]$, the limit being taken in the strict topology with $\theta \in C(\hat{\Gamma})$.

The proof of the theorem is arranged as follows. We start with a generalized Laplacian $D$. Then (i) uniquely determines a function $\nu$ which must be shown to be negative definite. The hard point of this step is the proof that $\nu$ is continuous. Next we demonstrate that if $\nu$ is negative definite, (ii) uniquely determines a symmetric probability semi-group. The essential point here is

(20) I am indebted to G. A. Hunt for pointing out the fundamental importance of the last condition. (The notation $D \circ \theta$, for $D$ operating on $\theta$, is used to emphasize the similarity with convolution.)

(21) Cf. Schoenberg $[2 ; 3]$.

(22) Statement (ii) is due to Schoenberg, loc. cit. (iii) is established by HuNT [1] for Lie groups; his methods depend strongly on the differential structure. 
that if $\nu$ is negative definite and $\xi \geqq 0$ then $\exp \{-\xi \nu\}$ is positive definite. Finally, having a symmetric probability semi-group acting on $C(\hat{\Gamma})$ we examine its infinitesimal generator. This is not only a generalized Laplace operator but is actually identical with the original $D$. The circle is thus complete.

Proof. Part I. Starting with $D$, a generalized Laplacian, we define $\nu(x)$ $=D \circ e_{x}(0)$. Since $D$ commutes with translations, $D \circ e_{x}=\nu(x) e_{x}$. The fact that $D$ is real and symmetric implies the same about $\nu$. Since constants attain their maximum and minimum at 0 , (D5) says in particular that generalized Laplacians annihilate constants; hence $\nu(0)=0$. Now let $\left\{c_{i}\right\}$ be a finite collection of real constants with $\sum c_{i}=0$. Then given a corresponding collection of points $\left\{x_{i}\right\}$ of $\Gamma, \theta(t)=\left|\sum c_{i} e\left(t x_{i}\right)\right|^{2}$ is in the range of $D$, since it is a trigonometric polynomial, and achieves its minimum at 0 . Application of (D5) yields (N2) directly. From the facts already at hand it is easy to deduce that $\nu(x) \geqq 0$ and $\nu^{1 / 2}(x+y) \leqq \nu^{1 / 2}(x)+\nu^{1 / 2}(y)$. (Since $\nu(0)=0$ we conclude that $\nu^{1 / 2}$ is an invariant pseudometric on $\Gamma$.) The preceding inequalities together with the statement that the graph of $\nu$ is closed, a direct consequence of (D1), suffice to establish the continuity of $\nu$. The reader will have no trouble convincing himself that a necessary and sufficient condition for a function on a locally compact Hausdorff space to be continuous is that the restriction of the function to any compact subset have a compact graph. Thus we have only to show that $\nu$ is bounded on compact sets; but, since $\nu^{1 / 2}$ is an invariant pseudometric, this will follow if we can show that $\nu$ is bounded on some nonvoid open set, say $\Omega$, for if $\mathrm{K}$ is compact there will exist a finite set $\Phi$ such that $\mathrm{K} \subset \Phi+\Omega$ and hence $\sup _{x \in \mathrm{K}} \nu^{1 / 2}(x) \leqq \sup _{y \in \Phi, z \in \Omega} \nu^{1 / 2}(y+z) \leqq \sup _{y \in \Phi} \nu^{1 / 2}(y)+\sup _{z \in \Omega} \nu^{1 / 2}(z)$ $<\infty$. Well, any non-negative function on a locally compact Hausdorff space which has a closed graph is lower semi-continuous. In particular, $\Gamma_{n}$ $=\{x \in \Gamma \mid \nu(x) \leqq n\}$ is closed. $\Gamma=\bigcup_{n=1}^{\infty} \Gamma_{n}$; so by Baire's category theorem, one of the $\Gamma_{n}$ 's must have a nonvoid interior and that is our $\Omega$.

Proof. Part II. If for each $\xi>0$ we have a probability measure $\pi(\xi)$, i.e. one satisfying (P1), with Fourier-Stieltjes transform $\exp \{-\xi \nu\}$ where $\nu$ satisfies (N1), then (P2)-(P4) follow quite easily. Since $\nu$ is continuous and $\nu(0)=0$ it will follow from Bochner's theorem that such $\pi(\xi)$ exist provided that for each finite collection of pairs $\left(c_{1}, x_{1}\right), \ldots,\left(c_{n}, x_{n}\right)$ where $c_{i}$ is a real number and $x_{i} \in \Gamma, \sum c_{i} c_{j} \exp \left\{-\xi \nu\left(x_{i}-x_{j}\right)\right\} \geqq 0$. To prove this, define an $n \times n$ matrix $X$ by

$$
2 X_{i, j}=\nu\left(x_{i}\right)+\nu\left(x_{j}\right)-\nu\left(x_{i}-x_{j}\right) .
$$

The assumption that $\nu$ is negative definite implies that $X$ is a positive definite quadratic form. Thus there exists an $n \times n$ matrix $Y$ with $X=Y Y^{\prime}$ where $Y^{\prime}$ is the transpose of $Y$. The rows of $Y$ may be considered as vectors $y_{1}, \cdots, y_{n}$ in Euclidean space $R^{n}$. The whole point is that $\left|y_{i}-y_{j}\right|^{2}=\nu\left(x_{i}-x_{j}\right),|y|$ being the ordinary Euclidean norm. The verification is straightforward: $\left|y_{i}-y_{j}\right|^{2}$ $=\sum_{k}\left(Y_{i, k}-Y_{j, k}\right)^{2}=\sum_{k} Y_{i, k}^{2}+\sum_{k} Y_{j, k}^{2}-2 \sum_{k} Y_{i, k} Y_{j, k}=X_{i, i}+X_{j, j}-2 X_{i, j}$ 
$=\nu\left(x_{i}-x_{j}\right)$. Now it is well known that for each $\xi \geqq 0, \exp \left\{-\xi|y|^{2}\right\}$ is a positive definite function on $R^{n}$. Hence

$$
\sum c_{i} c_{j} \exp \left\{-\xi \nu\left(x_{i}-x_{j}\right)\right\}=\sum c_{i} c_{j} \exp \left\{-\xi\left|y_{i}-y_{j}\right|^{2}\right\} \geqq 0 .
$$

Proof. Part III. Given a symmetric probability semi-group $\{\pi(\xi)\}$, it is obvious that the operation $D^{\prime}$ defined for $\theta \in C(\hat{\Gamma})$ by

$$
D^{\prime} \circ \theta=\lim \xi^{-1}[\theta-\pi(\xi) \circ \theta],
$$

the limit being taken in the strict topology as $\xi \rightarrow 0$, has all the properties of a generalized Laplacian except perhaps (D1) and agrees with the original $D$ on the characters. What remains to be proved is that $D^{\prime}$ is closed and that two generalized Laplacians which agree on the characters are identical. To establish the former, first observe that for the limit defining $D^{\prime}$ it is sufficient to take a sequence of $\xi^{\prime}$ s tending to $0 . \theta$ being in the domain of $D^{\prime}$ means that for each $\chi \in C_{0}(\widehat{\Gamma}),\left\|\left\{\xi^{-1}[\theta-\pi(\xi) \circ \theta]-D^{\prime} \circ \theta\right\} \chi\right\| \rightarrow 0$. Applying the Principle of Uniform Boundedness (BANACH, Theorem 5, p. 80) one sees that $\left\|\xi^{-1}[\theta-\pi(\xi) \circ \theta]\right\|$ is uniformly bounded. Now choose $\epsilon>0$ and define the transformation $D_{\epsilon}^{\prime}$ on the domain of $D^{\prime}$ by $D_{\epsilon}^{\prime} \circ \theta=D^{\prime} \circ \theta+\epsilon \theta$ $=\lim \xi^{-1}[\theta-\exp (-\epsilon \xi) \pi(\xi) \circ \theta]$, the limit again being in the strict topology. We intend to show that the resolvent $\left(D_{!}^{\prime}\right)^{-1}$ is represented by a kernel $\kappa \in B(\hat{\Gamma})$ where for any Borel set $\mathbf{B}, \kappa(\mathbf{B})=\int_{0}^{\infty} \exp (-\epsilon \eta) \pi(\eta ; \mathbf{B}) d \eta$. Given $\theta \in C(\hat{\Gamma}), \quad \xi^{-1}[\kappa \circ \theta-\exp (-\epsilon \xi) \pi(\xi) \circ \kappa \circ \theta]=\xi^{-1} \int_{0}^{\xi} \exp (-\epsilon \eta) \pi(\eta) \circ \theta d \eta$. This expression is uniformly bounded and $\exp (-\epsilon \eta) \pi(\eta) \circ \theta \rightarrow \theta$ as $\eta \rightarrow 0$ uniformly on compact sets. Hence the limit as $\xi \rightarrow 0$ exists in the strict topology and equals $\theta$, i.e. $D^{\prime} \circ \kappa$ is the identity transformation on $C(\hat{\Gamma})$. Conversely, if $\theta$ belongs to the domain of $D_{\epsilon}^{\prime}$ we can write

$$
\begin{aligned}
\kappa \circ D^{\prime} \circ \theta & =\kappa \circ \lim \xi^{-1}[\theta-\exp (-\epsilon \xi) \pi(\xi) \circ \theta] \\
& =\lim \xi^{-1}[\kappa \circ \theta-\exp (-\epsilon \xi) \pi(\xi) \circ \kappa \circ \theta]=\theta,
\end{aligned}
$$

the interchange of the limit and convolution with $\kappa$ being justified by uniform boundedness. This proves $\kappa \circ D_{\epsilon}^{\prime}$ is the identity on the domain of $D_{\epsilon}^{\prime}$. Since the convolution transform defined by $\kappa$ on $C(\hat{\Gamma})$ is continuous in the strict topology, its inverse $D_{\epsilon}^{\prime}$, and hence $D^{\prime}$, is closed. To complete the proof suppose $D$ is any generalized Laplacian which agrees with $D^{\prime}$ on the characters. Since $D$ is closed and commutes with translations, $\alpha \circ(D \circ \theta)=D \circ(\alpha \circ \theta)$ whenever $\alpha \in B(\hat{\Gamma})$ and $\theta$ is in the domain of $D$. Thus, in particular, $\kappa$ and $D_{\epsilon}$ commute on the domain of $D$, while for a trigonometric polynomial $\tau$, $\kappa \circ D_{\boldsymbol{\epsilon}} \circ \tau=\tau$. Suppose $\theta \in C(\hat{\Gamma})$ is arbitrary, and let $\{\tau\}$ be a directed set of trigonometric polynomials converging to $\theta$ in the strict topology (such a set exists because the trigonometric polynomials are strictly dense). Then $\kappa \circ \tau \rightarrow \kappa \circ \theta$ and $D_{\epsilon} \circ(\kappa \circ \tau)=\tau \rightarrow \theta$. Since $D_{\epsilon}$ is closed, $\kappa \circ \theta$ must be in the domain of $D$ and $D_{\epsilon} \circ(\kappa \circ \theta)=\theta$. Thus $D_{\epsilon}=\kappa^{-1}=D_{\epsilon}^{\prime}$, i.e. $D=D^{\prime}$. 
If we were to restrict our attention to $C_{0}(\hat{\Gamma})$, the strict topology could be replaced by the norm topology and ordinary semi-group theory could be applied. However, if we consider the adjoint semi-group, which is still represented by $\{\pi(\xi)\}$ acting by convolution, on $B(\hat{\Gamma})$, there will no longer be strong continuity in the norm topology. Nevertheless, the resolvent is still represented by the kernel $\kappa$ and is one-to-one since $\int e(t x) \kappa(d x)=q(x)$ $=[\epsilon+\nu(x)]^{-1} \neq 0$. One may define the adjoint generalized Laplacian, $D^{*}$, by specifying that $D_{\epsilon}^{*}$ is the inverse of the convolution transform with kernel $\kappa$ of $B(\hat{\Gamma})$ into itself. Actually $D^{*}$ is the appropriate infinitesimal generator; to apply semi-group theory one need only cut down $B(\hat{\Gamma})$ to the closure of the union of the ranges of $\pi(\xi), \xi>0$. The potential theory needed here is one in which $D^{*}$ is of direct concern rather than $D$.

Definition. $\alpha \in B(\Gamma)$ is $D_{e}^{*}$-superharmonic if $\alpha$ belongs to the domain of $D^{*}$ and $D_{\text {c }}^{*} \circ \alpha \geqq 0$.

That is to say, $\alpha$ is superharmonic if $\beta=D_{c}^{*} \circ \alpha$ is a positive chargedistribution, which by the definition of $D_{e}^{*}$ means that $\alpha=\kappa \circ \beta$, i.e. $\alpha$ is the potential of a positive measure. All this would be banal were it not for the fact that superharmoniciţy is equivalent to a mean value property.

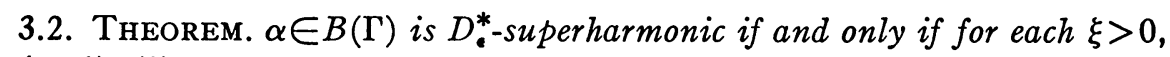
$\exp (-\epsilon \xi) \pi(\xi) \circ \alpha \leqq \alpha$.

Proof. Let $\phi$ be the Fourier-Stieltjes transform of $\alpha$. The statement that $\exp (-\epsilon \xi) \pi(\xi) \circ \alpha \leqq \alpha$ is equivalent to the statement that $\phi \cdot[1-\exp \{-\xi(\epsilon+\nu)\}]$ is positive definite. Now as $\xi \rightarrow 0, \xi^{-1} \phi \cdot[1-\exp \{-\xi(\epsilon+\nu)\}]$ converges uniformly on compact sets to $\psi=\phi \cdot(\epsilon+\nu)$. According to the Lévy convergence theorem, $\psi$ is also positive definite, i.e. $\psi(x)=\int e(t x) d \beta(t)$ where $\beta$ is a nonnegative measure on $\hat{\Gamma}$. Since $\phi=q \psi, \alpha=\kappa \circ \beta$. The converse is entirely trivial: if $\alpha=\kappa \circ \beta$ and B is any Borel set then

$$
\begin{aligned}
\alpha(\mathrm{B})- & \exp (-\epsilon \xi) \pi(\xi) \circ \alpha(\mathrm{B})=\int_{0}^{\infty} \exp (-\epsilon \eta) \pi(\eta) \circ \beta(\mathrm{B}) d \eta \\
& -\int_{0}^{\infty} \exp \{-\epsilon(\xi+\eta)\} \pi(\xi+\eta) \circ \beta(\mathrm{B}) d \eta=\int_{0}^{\xi} \exp (-\epsilon \eta) \pi(\eta) \circ \beta(\mathrm{B}) d \eta
\end{aligned}
$$

which is certainly non-negative if $\beta$ is.

The importance of the previous theorem lies in the obvious corollary that the greatest lower bound of two $D_{e}^{*}$-superharmonic measures is again $D_{\epsilon}^{*}$ superharmonic. This fact implies the "Principle of the Maximum" for our potential theory $\left({ }^{23}\right)$.

(23) Cartan gives a lucid exposition of the matters of concern here. However he puts the semi-group in the "wrong" place. The importance of the mean value property is stressed in CARTAN and DENy, but again the conditions on the kernel there are not quite the "right" ones. HunT [2] clarifies the whole problem. One could subsume the results here under his work by assuming the second axiom of countability, but there is so much structure present in our case that it is easier to do things anew than to force them into another mold. 
To proceed, the concept of "Energy" is needed. For $\alpha \in B(\hat{\Gamma})$ with FourierStieltjes transform $\varphi$, the energy, $\varepsilon(\alpha)$, of the charge distribution $\alpha$, associated with the potential theory having $\kappa$ for kernel, is defined to be $\mathcal{E}(\alpha)=2^{-1}$ $\int|\varphi(x)|{ }^{2} q(x) d x=2^{-1} Q(\varphi, \varphi)$. In case $\kappa$ has a density, $\hat{q}, \quad \varepsilon(\alpha)=2^{-1}$ $\int \hat{q}(s-t) \alpha(d t) \bar{\alpha}(d s)$. The important thing is that the only charge distribution with zero-energy is the zero element of $B(\hat{\Gamma})$ while on the other hand there are a great many distributions with finite energy, e.g. any distribution with a square-integrable density.

If one completes the charge-distributions of finite energy to form a Hilbert space under the norm-energy, then on going over to the Fourier transform he has the space $L_{q}^{2}$ of equivalence classes of measurable functions $\varphi$ on $\Gamma$ with $\int|\varphi(x)|^{2} q(x) d x<\infty$. The inner product will be written as $Q(\varphi, \psi)$ $=\left(\varphi q, \psi^{*}\right)$. Actually, if we were not concerned with the interpretation, we could have started out with a function $q$ on $\Gamma$ of the form $q(x)=[\epsilon+\nu(x)]^{-1}$, where $\epsilon$ is a positive number and $\nu$ a negative definite function, formed the Hilbert space $L_{q}^{2}$, and then proceeded as below. The connection between potential theory and spectral analysis involves simply the study of $L_{q}^{2}$.

The first part of the proof of (3.1) showed that $\nu^{1 / 2}$ was a continuous invariant pseudometric on $\Gamma$. The same is true of the function $\delta=2 \log \left(1+\nu^{1 / 2}\right)$. Now, in general, given any pseudometric $\delta$ (the property $\delta(x+y) \leqq \delta(x)+\delta(y)$ is the essential one here), the set of measurable functions $f$ on $\Gamma$ with the norm $\int|f(x)| \exp \{\delta(x)\} d x$ forms a Banach algebra under convolution. These "Beurling algebras" have been studied $\left({ }^{24}\right)$, and it turns out that if, for each $x \in \Gamma, \sum_{1}^{\infty} n^{-2} \delta(n x)<\infty$ then we have to do with a regular, semi-simple Banach algebra with involution whose maximal ideal space is simply $\hat{\Gamma}$. The $\delta$ given at the beginning of this paragraph fulfills the condition; a translation of the previous sentence yields

3.3. Lemma. Given a compact set $\mathrm{T} \subset \hat{\Gamma}$ and a nucleus $\mathrm{N}$ there is an $f \in L^{1}(\Gamma)$ such that $\int|f(x)| \nu(x) d x<\infty, \hat{f}(t)=1$ for $t \in \mathrm{T}, \hat{f}(s)=0$ for $s \notin \mathrm{T}+\mathrm{N}$, and ever $y$ where $0 \leqq \hat{f} \leqq 1$.

The lemma suggests an extension of the notion of spectrum to $L_{q}^{2}$. Indeed, given a subset $\mathrm{T}$ of $\hat{\Gamma}$, let $P_{\mathrm{T}}$ denote the set of functions of the form $\varphi(x)=\int e(t x) \alpha(d t)$ where $\alpha$ is a positive charge distribution of finite energy with $\Sigma(\alpha) \subset \mathrm{T}$, and further let $Q_{\mathrm{T}}$ denote the set of all $\varphi \in C(\Gamma) \cap L_{q}^{2}$ with $\Lambda(\varphi) \subset \mathrm{T}$. In other words $Q_{\mathrm{T}}=E^{\prime}(\mathrm{T}) \cap L_{q}^{2}$ and $P_{\mathrm{T}}$ is the set of positive definite functions in $Q_{\mathrm{T}}$. Observe that no special notation is introduced for $E(\mathrm{~T}) \cap L_{q}^{2}$; that is a clue, - at the end of the story, Theorem 3.6, we shall find that this set is all of $Q_{\mathrm{T}}$. To get on, $\bar{P}_{\mathrm{T}}$ and $\bar{Q}_{\mathrm{T}}$ are the closures of the respective sets in $L_{q}^{2}$. The point of the lemma is that, roughly speaking, these closures don't add anything new.

(24) Beurling [3] and Domar. 
3.4. Theorem. If $\mathrm{T}$ is closed, $\bar{Q}_{\mathrm{T}} \cap C(\Gamma)=Q_{\mathrm{T}}$. Further, if $\mathrm{T}$ is compact, $\bar{P}_{\mathrm{T}}=P_{\mathrm{T}}$.

Proof. The first sentence of the theorem is an obvious corollary of (3.3) (the extension from compact $\mathrm{T}$ to closed $\mathrm{T}$ is straightforward), but anyhow let us take T compact and $f$ as in the lemma except now assume $\hat{f}=1$ on a neighborhood of $\mathrm{T}$. Then clearly, for $\varphi \in Q_{\mathrm{T}}, \varphi \rightarrow \varphi(0)=(\varphi, f)$ is a bounded linear functional. The square of the norm of this functional is a constant $C(\mathrm{~T})$ called "the $D_{\epsilon}$-capacity of T." (The justification for this terminology will become more apparent later on.) Thus $|\varphi(0)|^{2} \leqq C(\mathrm{~T}) Q(\varphi, \varphi)$. Using the pseudometric property of $\nu^{1 / 2}$ and the fact that $Q_{\mathrm{T}}$ is invariant under translation, we obtain for all $\varphi \in Q_{\mathrm{T}}$ and $x \in \Gamma$

$$
|\varphi(x)|^{2} \leqq C(\mathrm{~T})\left\{1+\epsilon^{-1 / 2} \nu^{1 / 2}(x)+e^{-1} \nu(x)\right\} \int|\varphi(y)|^{2}[\epsilon+\nu(y)]^{-1} d y .
$$

This implies that a sequence of functions of $Q_{T}$ which converges in the mean of $L_{q}^{2}$ also converges uniformly on compact sets. A corollary, via the Lévy convergence theorem, is that $P_{\mathrm{T}}$ is closed.

Next comes the key result. Its statement will be strengthened later on, so it appears only as a lemma.

3.5. Lemma. Let $\varphi$ be a positive definite function in $L_{a}^{2}$ and $\mathrm{T}$ a given compact subset of $\hat{\Gamma}$. Define $\varphi_{\mathrm{T}}$ to be the orthogonal projection of $\varphi$ on $\bar{Q}_{\mathrm{T}}$. Then

(i) $\varphi_{\mathrm{T}} \in P_{\mathrm{T}}$,

(ii) for each positive definite $\psi \in L_{q}^{2}, Q\left(\varphi_{\mathrm{T}}, \psi\right) \leqq Q(\varphi, \psi)$.

The demonstration is somewhat round-about. In Part I we define a function, denoted as $\varphi_{\mathbf{T}}$, which satisfies (i) and (ii); in Part II it is shown that this function is actually the orthogonal projection of $\varphi$ on $\bar{Q}_{\mathrm{T}}$.

Proof. Part I( $\left.{ }^{25}\right)$. Since $P_{\mathrm{T}}$ is a closed convex set in $L_{q}^{2}$ there exists a unique element, call it $\varphi_{\mathrm{T}}$, of $P_{\mathrm{T}}$ which is closest to $\varphi$. $P_{\mathrm{T}}$ being a cone, for each $\psi \in P_{\mathrm{T}}, Q(\varphi, \psi) \leqq Q\left(\varphi_{\mathrm{T}}, \psi\right)$ while $Q\left(\varphi, \varphi_{\mathrm{T}}\right)=Q\left(\varphi_{\mathrm{T}}, \varphi_{\mathrm{T}}\right) . \varphi$ and $\varphi_{\mathrm{T}}$ are respectively the Fourier-Stieltjes transforms of positive charge distributions of finite energy $\alpha$ and $\alpha_{\mathrm{T}}$. By (3.2), the greatest lower bound of the potentials $\kappa \circ \alpha$ and $\kappa \circ \alpha_{\mathrm{T}}$ is again a potential $\kappa \circ \beta$, where $\beta$ is a positive charge distribution which is also of finite energy. Let $\omega$ be the Fourier-Stieltjes transform of $\beta$. It follows from the definition of the greatest lower bound of two measures that for each positive definite $\psi \in L_{q}^{2},(\omega q, \psi)=\inf \left[\left(\varphi q, \psi_{1}\right)+\left(\varphi_{\mathrm{T}} q, \psi_{2}\right)\right]$, the infimum being taken over all decompositions $\psi=\psi_{1}+\psi_{2}$ with $\psi_{1}$ and $\psi_{2}$ positive definite. In the notation of the inner product on $L_{q}^{2}$ the above is $Q(\omega, \psi)$ $=\inf \left[Q\left(\varphi, \psi_{1}\right)+Q\left(\varphi, \psi_{2}\right)\right]$. Thus for all positive definite $\psi \in L_{q}^{2}, Q\left(\varphi_{\mathrm{T}}-\omega, \psi\right)$ $\geqq 0$; on the other hand $Q\left(\omega, \varphi_{\mathrm{T}}\right)=Q\left(\varphi_{\mathrm{T}}, \varphi_{\mathrm{T}}\right)$. This implies $\varphi_{\mathrm{T}}=\omega$, for $Q\left(\varphi_{\mathrm{T}}-\omega, \varphi_{\mathrm{T}}-\omega\right)=Q\left(\varphi_{\mathrm{T}}-\omega, \varphi_{\mathrm{T}}\right)-Q\left(\varphi_{\mathrm{T}}-\omega, \omega\right) \leqq 0$ since the minuend is zero and the subtrahend non-negative. Therefore our $\varphi_{\mathrm{T}}$ satisfies both (i) and (ii).

(25) Cf. Cartan. 
Proof. Part II. If $\psi \in P_{\mathrm{T}}$, we know that $Q(\varphi, \psi) \leqq Q\left(\varphi_{\mathrm{T}}, \psi\right)$. However $Q(\varphi, \psi) \geqq Q(\omega, \psi)=Q\left(\varphi_{\mathrm{T}}, \psi\right)$, so that equality holds. We conclude that $Q(\varphi, \psi)$ $=Q\left(\varphi_{\mathrm{T}}, \psi\right)$ for each $\psi$ in the closed linear manifold spanned by $P_{\mathrm{T}}$; what remains to be proved is that the equation is valid for each $\psi \in Q_{\mathrm{T}}$. To this purpose, choose a $J$-net $\{\mathrm{H}\}$ and let $\{\mathrm{A}\}$ be the collection of sets of the form $\mathbf{A}=\mathbf{T}+\overline{\mathrm{H}}^{0}$. The above arguments apply to the compact sets $\mathrm{A}$; so if $\mathbf{A} \subset \mathbf{B}$, $Q\left(\varphi_{\mathrm{B}}, \varphi_{\mathrm{A}}\right)=Q\left(\varphi, \varphi_{\mathrm{A}}\right)=Q\left(\varphi_{\mathrm{A}}, \varphi_{\mathrm{A}}\right)$. Hence $Q\left(\varphi_{\mathrm{B}}-\varphi_{\mathrm{A}}, \varphi_{\mathrm{B}}-\varphi_{\mathrm{A}}\right)=Q\left(\varphi_{\mathrm{B}}, \varphi_{\mathrm{B}}\right)$ $-Q\left(\varphi_{A}, \varphi_{A}\right)$. Since $Q\left(\varphi_{A}, \varphi_{A}\right)$ is non-negative and decreases with $A$ by the preceding equation, $\varphi_{\mathrm{A}}$ must be a Cauchy sequence in $L_{q}^{2}$. Let $\theta$ be the limit. $\theta \in \cap P_{\mathrm{A}}$, but $\cap \mathrm{A}=\mathrm{T}$; thus $\theta \in P_{\mathrm{T}}$. Moreover, if $\psi \in P_{\mathrm{T}}, Q(\theta, \psi)=\lim Q\left(\varphi_{\mathrm{A}}, \psi\right)$ $=Q(\varphi, \psi)$. Therefore $\theta=\varphi_{\mathrm{T}}$. Now suppose $\psi \in Q_{\mathrm{T}}$; then $k_{\mathrm{H}} \psi$ belongs to the linear manifold spanned by $P_{\mathbf{A}}$ whenever $\mathrm{T}+\overline{\mathrm{H}} \subset \mathrm{A}$. For fixed $\mathrm{A}$, one has $Q(\varphi, \psi)=\lim _{\mathrm{H} \rightarrow 0} Q\left(\varphi, k_{\mathrm{H}} \psi\right)=\lim _{\mathrm{H} \rightarrow 0} Q\left(\varphi_{\mathrm{A}}, k_{\mathrm{H}} \psi\right)=Q\left(\varphi_{\mathrm{A}}, \psi\right)$. Taking the limit as $\mathrm{A} \rightarrow \mathrm{T}$ we get $Q(\varphi, \psi)=Q\left(\varphi_{\mathrm{T}}, \psi\right)$ which completes the demonstration.

As an immediate consequence of the above, one has the basic theorem connecting potential theory with spectral theory.

3.6. Theorem. For any closed set $\mathrm{T}$ in $\Gamma, P_{\mathrm{T}}$ is a fundamental subset of $\bar{Q}_{\mathrm{T}}$.

Proof. One has only to note that if $\psi \in L_{q}^{2}$ and $Q(\varphi, \psi)=0$ for each positive definite $\varphi \in L_{q}^{2}$ then $\psi=0$. If $\mathrm{T}$ is compact the theorem is an immediate consequence of (3.5). Otherwise one uses (3.3) to reduce the problem to consideration of functions with compact spectrum, and (3.5) shows that $U P_{\Sigma}$ is fundamental in $\bar{Q}_{\mathbf{T}}$ where the union is taken over all compact $\Sigma \subset \mathrm{T}$.

In order to give (3.6) a more concrete interpretation for spectral analysis, it is necessary to investigate the notion of capacity. Recall that for compact $\mathrm{T}$ we have proved that $\varphi \rightarrow \varphi(0)$ is a bounded linear functional on $Q_{\mathrm{T}}$; the square of the norm of this functional was the capacity $C(\mathrm{~T})$. Thus there is an $\omega_{\mathrm{T}} \in \bar{Q}_{\mathrm{T}}$ such that $\varphi(0)=Q\left(\varphi, \omega_{\mathrm{T}}\right)$.

3.7. Lemma. Let $\mathrm{T}$ be a given compact subset of $\hat{\Gamma}$. Define $\omega_{\mathrm{T}}$ to be the element of $\bar{Q}_{\mathrm{T}}$ such that $\varphi(0)=Q\left(\varphi, \omega_{\mathrm{T}}\right)$ for each of $\varphi \in \bar{Q}_{\mathrm{T}}$. Then

(i) $\omega_{\mathrm{T}} \in P_{\mathrm{T}}$,

(ii) for each positive definite $\psi \in L_{a}^{2}, Q\left(\omega_{\mathrm{T}}, \psi\right) \leqq \psi(0)$.

This resembles (3.5) very closely. Indeed, if $\omega$ were $\epsilon$ times a $\delta$-function and $\omega_{T}$ its orthogonal projection on $\bar{Q}_{\mathrm{T}}$ the statements would be precisely analogous. The last sentence may serve as a sufficient indication of how to prove the lemma.

The above $\omega_{\mathrm{T}}$ is the Fourier-Stieltjes transform of a finite, positive charge distribution, $\gamma_{\mathrm{T}}$, the equilibrium distribution on T, with $\Sigma\left(\gamma_{\mathrm{T}}\right) \subset \mathrm{T}$. It is clear that $C(\mathrm{~T})=\gamma_{\mathrm{T}}(\mathrm{T})=2 \varepsilon\left(\gamma_{\mathrm{T}}\right)$. The potential of $\gamma_{\mathrm{T}}, \kappa \circ \gamma_{\mathrm{T}}$, is the equilibrium potential of T. (3.7) (ii) is equivalent to the statement $\kappa \circ \gamma_{\mathbf{T}}(\mathrm{B}) \leqq|\mathrm{B}|$ for each Borel subset $B$ of $\hat{\Gamma}$. This observation enables us to formulate the definition of capacity in a natural way, i.e. the way one would define the capacity of a condenser, without reference to energy. 
Definition $\left({ }^{26}\right)$. The capacity of a compact set $\mathrm{T}$ in $\hat{\mathrm{T}}$ for the potential theory with kernel $\kappa$ is $\sup \alpha(\mathrm{T})$ where the supremum is taken over all positive charge distributions $\alpha$ with spectrum in $\mathrm{T}$ whose potentials are dominated by the Haar measure, i.e. $\kappa \circ \alpha(\mathrm{B}) \leqq|\mathrm{B}|$ for each Borel set $\mathrm{B}$.

3.8. Theorem. If $\alpha$ is any finite positive charge distribution with $\Sigma(\alpha) \subset \mathbf{T}$ and $\kappa \circ \alpha \leqq H a a r$ measure, then $\kappa \circ \alpha \leqq \kappa \circ \gamma_{\mathrm{T}}$ and $\alpha(\mathrm{T}) \leqq \gamma_{\mathrm{T}}(\mathrm{T})$ where $\gamma_{\mathrm{T}}$ is the equilibrium distribution on $\mathrm{T}$. Thus the capacity of $\mathrm{T}$ is $C_{\mathrm{\epsilon}}(\mathrm{T})$ $=\left\{\inf \int|\varphi(x)|{ }^{2}[\epsilon+\nu(x)]^{-1} d x\right\}^{-1}$ where the infimum is taken over all $\varphi \in C(\Gamma)$ with $\Lambda(\varphi) \subset \mathrm{T}$ and $\varphi(0)=1$.

Proof. Since $0 \leqq \kappa \circ \alpha \leqq$ Haar measure, we have for the Fourier-Stieltjes transform, $\varphi$, of $\alpha$ that $0 \leqq Q(\psi, \varphi) \leqq \psi(0)$ for all positive definite $\psi$ where $Q(\psi, \varphi)$ is interpreted in some generalized sense. However, since $\Sigma(\alpha)$ is compact one actually has $\varphi \in P_{\mathrm{T}}$ and the above inequality may be taken literally for $\psi \in L_{q}^{2}$. Accordingly we have for each positive definite $\psi \in L_{q}^{2} Q\left(\psi, \omega_{\mathrm{T}}-\varphi\right)$ $=Q\left(\psi_{\mathrm{T}}, \omega_{\mathrm{T}}-\varphi\right)=\psi_{\mathrm{T}}(0)-Q\left(\psi_{\mathrm{T}}, \varphi\right) \geqq 0$ which is equivalent to $\kappa \circ \gamma_{\mathrm{T}} \geqq \kappa \circ \alpha$. Moreover, taking $\psi=\omega_{\mathrm{T}}$ we get $\gamma_{\mathrm{T}}(\mathrm{T})-\alpha(\mathrm{T})=Q\left(\omega_{\mathrm{T}}, \omega_{\mathrm{T}}-\varphi\right) \geqq 0$. We have previously proved that $\gamma_{\mathrm{T}}(\mathrm{T})=C_{\mathrm{e}}(\mathrm{T})$.

It remains to justify the use of the word "capacity." We have in mind the general theory of CHOQUET; what is true is that $C(\mathrm{~T})$ is a non-negative, alternating capacity of order $\infty$ on the class of compact subsets of $\hat{\Gamma}$. An extensive discussion of the properties of such functions is given by CHOQUET. To shorten the terminology we shall call them "ordinary capacities."

Definition. A real valued function $F$ defined on all compact subsets of a locally compact Hausdorff space is an "ordinary capacity" if

(C1) $F(\varnothing)=0$,

(C2) given $\mathrm{T}$ compact and $a>0$ there exists a neighborhood $\Omega_{a}$ of $\mathrm{T}$ such that if $\mathbf{A}$ is compact and $\mathbf{A} \subset \Omega_{a}$ then $F(\mathbf{A})<F(\mathbf{T})+a$;

(C3) if one defines $F^{(1)}\left(\mathrm{T} ; \mathrm{A}_{1}\right)=F\left(\mathrm{~T} \cup \mathrm{A}_{1}\right)-F(\mathrm{~T})$ and inductively $F^{(n+1)}\left(\mathrm{T} ; \mathrm{A}_{1}, \cdots, \mathrm{A}_{n+1}\right)=F^{(n)}\left(\mathrm{T} ; \mathrm{A}_{1}, \cdots, \mathrm{A}_{n}\right)-F^{(n)}\left(\mathrm{T} \cup \mathrm{A}_{n+1} ; \mathrm{A}_{1}, \cdots, \mathrm{A}_{n}\right)$, then $F^{(n)}\left(\mathbf{T} ; \mathbf{A}_{1}, \cdots, \mathbf{A}_{n}\right) \geqq 0$ for all compact $n+1$-tuples $\mathbf{T}, \mathbf{A}_{1}, \cdots, \mathbf{A}_{n}$.

3.5'. TheOREM. Let $\alpha$ be a finite positive charge distribution of finite energy. If $\mathrm{T}$ is compact, there is a unique non-negative charge distribution $\alpha_{\mathrm{T}}$, the "sweeping-out" of $\alpha$ onto $\mathrm{T}$ which among all charge distributions $\beta$ with $\Sigma(\beta) \subset \mathrm{T}$ minimizes the quantity $\&(\alpha-\beta)$. Also $\left\|\alpha_{\mathrm{T}}\right\| \leqq\|\alpha\|$. For a fixed Borel set $\mathrm{B}$ and variable compact $\mathrm{T}$, set $F_{\mathrm{B}}(\mathrm{T})=\kappa \circ \alpha_{\mathrm{T}}(\mathrm{B})$; then $F_{\mathrm{B}}(\mathrm{T}) \leqq \kappa \circ \alpha(\mathrm{B})$ and $F_{\mathrm{B}}$ is an ordinary capacity.

Proof. Let $\varphi$ be the Fourier-Stieltjes transform of $\alpha . \varphi_{\mathrm{T}}$ as defined in (3.5)

(26) Capacity is defined by various writers with various normalizations. In the classical theory one usually has $\epsilon=0$; Deny has shown in unpublished work that one can take $\epsilon=0$ if and only if $\int_{N} \nu^{-1}(x) d x<\infty$ for some nucleus $\mathrm{N}$ in $\Gamma$. However, the essential point is whether the capacity is positive or zero and this does not depend on $\epsilon$ or the normalization. 
determines $\alpha_{\mathrm{T}} .\left\|\alpha_{\mathrm{T}}\right\|=\varphi_{\mathrm{T}}(0)=Q\left(\varphi, \omega_{\mathrm{T}}\right) \leqq \varphi(0)=\|\alpha\|$. For positive definite $\psi \in L_{a}^{2}$ set $F(\mathrm{~T} ; \psi)=Q\left(\varphi_{\mathrm{T}}, \psi\right)$. The proof is completed when we show that for each such $\psi, F(\mathrm{~T} ; \psi) \leqq Q(\varphi, \psi)$ and as a function of $\mathrm{T}, F(\mathrm{~T} ; \psi)$ is an ordinary capacity. The inequality is (3.5) (ii); property (C1) is trivial and (C2) was verified in Part II of the proof of (3.5). In order to demonstrate (C3) we shall prove by induction on $n$ : for each positive definite $\psi \in L_{q}^{2}$, $0 \leqq F^{(n)}\left(\mathrm{T} ; \mathrm{A}_{1}, \cdots, \mathrm{A}_{n} ; \psi\right)$ with equality for $\psi \in P_{\mathrm{T}}$. The case $n=1$ is straightforward; suppose now the statement is true for $n$. First, if

$$
\begin{aligned}
& \psi \in P_{\mathrm{T}}, F^{(n+1)}\left(\mathrm{T} ; \mathrm{A}_{1}, \cdots \mathrm{A}_{n+1} ; \psi\right) \\
& \quad=F^{(n)}\left(\mathrm{T} ; \mathrm{A}_{1}, \cdots, \mathrm{A}_{n} ; \psi\right)-F^{(n)}\left(\mathrm{T} \cup \mathrm{A}_{n+1} ; \mathrm{A}_{1}, \cdots, \mathrm{A}_{n} ; \psi\right)=0,
\end{aligned}
$$

by the inductive hypothesis since $P_{\mathrm{T}} \subset P_{\mathrm{TUA}_{n+1}}$. The same equation shows that $F^{(n+1)}\left(\mathrm{T} ; \mathrm{A}_{1}, \cdots, \mathrm{A}_{n+1} ; \psi\right) \geqq 0$ if $\psi \in P_{\mathrm{TUA}_{n+1}}$. On account of the symmetry in the $A_{i}$ 's the same inequality holds for $\psi \in P_{\text {TUA }_{i}}$. Now suppose $\psi \in P_{\mathbf{A}}$ where $\mathrm{A}=\mathrm{T} \cup \cup_{i=1}^{n+1} \mathrm{~A}_{i}$. Such a $\psi$ is the Fourier-Stieltjes transform of a positive charge distribution $\beta$ with $\Sigma(\beta) \subset \mathrm{A}$. We can decompose $\beta$ as $\beta=\sum \beta_{i}$ where $\Sigma\left(\beta_{i}\right) \subset \mathbf{T} \cup \mathrm{A}_{i}$ and each $\beta_{i}$ likewise is a positive charge distribution (of finite energy). Thus $\psi=\Sigma \psi_{i}$ and by linearity we have

$$
F^{(n+1)}\left(\mathrm{T} ; \mathrm{A}_{1}, \cdots, \mathrm{A}_{n+1} ; \psi\right) \geqq 0 \quad \text { for } \psi \in P_{\mathrm{A}} .
$$

Now suppose $\psi$ is an arbitrary positive definite function in $L_{q}^{2}$.

$$
F^{(n+1)}\left(\mathrm{T} ; \mathrm{A}_{1}, \cdots, \mathrm{A}_{n+1} ; \psi\right)=Q(\theta, \psi)
$$

where the precise form of $\theta$ is irrelevant but $\theta \in P_{\mathrm{A}}$. Thus $Q(\theta, \psi)=Q\left(\theta, \psi_{\mathrm{A}}\right)$, i.e. $F^{(n+1)}\left(\mathrm{T} ; \mathrm{A}_{1}, \cdots, \mathrm{A}_{n+1} ; \psi\right)=F^{(n+1)}\left(\mathrm{T} ; \mathrm{A}_{1}, \cdots, \mathrm{A}_{n+1} ; \psi_{\mathrm{A}}\right) \geqq 0$.

\subsection{Corollary. $C_{\epsilon}(\mathrm{T})$ is an ordinary capacity.}

Proof. At each stage of verification of the defining properties of an ordinary capacity we have to deal only with subcompacts of some compact set $K$. Let $K$ be given and let $T$ vary over compact subsets of $K$. Note that $C_{\epsilon}(\mathrm{T})=\epsilon \kappa \circ \gamma_{\mathrm{T}}(\hat{\Gamma}) ;(3.9)$ follows from $\left(3.5^{\prime}\right)$ by taking $\alpha=\epsilon \gamma_{\mathrm{K}}$.

At this point we have established those facts of potential theory which are directly relevant to our purposes. However, the theory by no means stops here, and we shall briefly mention two lines of further investigation. In the first place theorem $\left(3.5^{\prime}\right)$ remains true with the hypothesis of positivity omitted provided that one changes the final sentence to read " $\kappa \circ \alpha_{\mathrm{T}}(\mathrm{M})$ $=\kappa \circ \alpha(\mathrm{M})$ for each Haar measurable M $\subset$ T." Thus one has the balayage theorem for all charge distributions of finite energy. Unfortunately the statement in quotation marks above may be trivial, e.g. if $\mathrm{T}$ has measure 0 . This leads one to seek a finer potential theory; for example if $\kappa$ has a lower semicontinuous density $\hat{q}$, the potentials of measure can be considered as functions and may be investigated at each point $\left({ }^{27}\right)$. Even without this assumption,

(27) See Cartan and Deny. 
it is evident from the Fourier transform theory that the potentials are defined up to sets of capacity zero rather than up to sets of measure zero as our notation might indicate. Also, with additional assumptions one can free potential theory from the notion of energy and obtain a balayage theorem for all positive charge distributions. The second type of question concerns the nature of negative definite functions. A general representation theorem for negative definite functions is known only in the classical case $\left.{ }^{28}\right), \Gamma=I^{r_{1}} \oplus R^{r^{2}}$. Here the functions $\nu(x)=|x|^{p}, 0<p \leqq 2$, are the simplest examples, and a special consequence of (3.8) is worth quoting.

3.10. TheOREM $\left({ }^{29}\right)$. Let $\Gamma=I^{r_{1}} \oplus R^{r^{2}}, \quad r_{1}+r_{2}=r$, and suppose $0 \leqq p \leqq \min (2, r)$. Then $\varphi \in C(\Gamma)$ and $\int|\varphi(x)|^{2}\left(1+|x|^{p}\right)^{-1} d x<\infty$ imply that the Hausdorf dimension of $\Lambda(\varphi)$ is at least $r-p$. (If $r-p=0, \Lambda(\varphi)$ has at least logarithmic dimension.)

Finally we shall give a nonclassical example which will be used later.

ExAmple. A continuous invariant pseudometric $\nu$ on $\Gamma$ which has the property that $\nu(x+y) \leqq \max [\nu(x), \nu(y)]$ is called an "ultrametric." We proceed to show that an ultrametric is negative definite and to construct the corresponding probability semi-group and generalized Laplacian. Given $r>0$, let $\mathbf{B}_{r}=\{x \in \Gamma \mid \nu(x)<r\}$. $\mathbf{B}_{r}$ is an open subgroup of $\Gamma$; hence $\mathbf{B}_{r}^{\perp}$ is a compact subgroup of $\hat{\Gamma}$. Let $\beta(r)$ be the normalized Haar measure on $\mathrm{B}_{r}^{\perp}$; i.e. $\int \theta(t) \beta(r ; d t)$ is simply the average of $\theta$ on $\mathrm{B}_{r}^{\perp}$. We set

$$
\pi(\xi)=\xi \int_{0+}^{\infty} \exp (-\xi r) \beta(r) d r .
$$

It is easy to see that $\{\pi(\xi)\}$ satisfies (P1), (P2), and (P4). To verify (P3) note that $\beta(r) \circ \beta(s)=\beta[\min (r, s)]$. Thus

$$
\pi(\xi) \circ \pi(\eta)=\xi \eta \int_{0+}^{\infty} \int_{0+}^{\infty} \exp (-\xi r) \exp (-\eta s) \beta[\min (r, s)] d r d s=\pi(\xi+\eta) .
$$

The generalized Laplacian is given by

$$
D=\int_{0+}^{\infty}\{\epsilon-\beta(r)\} d r
$$

where the integral is to be interpreted as a strong Abel limit, i.e.

$$
D \circ \theta=\lim _{\xi \rightarrow 0} \int_{0+}^{\infty} \exp (-\xi r)\{\theta-\beta(r) \circ \theta\} d r .
$$

Choosing $\theta=e_{x}$ one has $D \circ e_{x}(0)=\int_{0+}^{\infty}\left\{1-\beta(r) \circ e_{x}(0)\right\} d r . \beta(r) \circ e_{x}(0)$ is the

(28) See Bochner [2]; a generalization is given in HuNT [1].

(29) Proved for $r=1$ in Beurling [5], for $r>1$ by Deny. 
average of $e_{x}$ over $\mathrm{B}_{r}^{\perp}$ and is 1 if $x$ is a trivial character on $\mathrm{B}_{r}^{\perp}$, i.e. if $\nu(x)<r$, and 0 otherwise. Thus $D \circ e_{x}(0)=\int_{0}^{\nu(x)} 1 d r=\nu(x)$. The standard representation is $\nu(x)=\int\{1-e(t x)\} \alpha(d t)$ where $\alpha=\int_{0+}^{\infty} \beta(r) d r$.

4. The spectral synthesis problem. The preceding sections have been concerned with spectral analysis, that is assigning to a given function $\varphi \in C(\Gamma)$ a certain set of characters $e_{t}, t \in \Lambda(\varphi)$. The converse problem is that of recovering, in some fashion, the original function from this set of characters. We say that spectral synthesis holds when $\varphi \in E(\Lambda(\varphi))$. This is always true when $\Gamma$ is compact $\left({ }^{30}\right)$, but there are examples showing that it may not hold in general for a given noncompact group. We shall say that spectral synthesis holds for a closed set $\mathbf{T} \subset \hat{\Gamma}$, or that $\mathrm{T}$ is an $S$-set, if $E^{\prime}(\mathrm{T})=E(\mathrm{~T})$. It is easy to see that $\mathrm{T}$ is an $S$-set if and only if the smallest closed ideal, $I^{\prime}(\mathrm{T})$, of the convolution algebra $L^{1}(\Gamma)$ containing all functions $f$ with $\mathrm{T} \subset \operatorname{Int} \mathrm{Z}(f)$ is the intersection of the regular maximal ideals containing it, i.e. if $g \in L^{1}(\Gamma)$ and $\mathrm{T} \subset \mathrm{Z}(\mathrm{g})$ imply $\mathrm{g} \in I^{\prime}(\mathrm{T})$.

Later on we shall investigate some special sets with regard to spectral synthesis. At this point we only remark that whether or not $\mathrm{T}$ is an $S$-set is a local property of $\mathrm{T}$ by which we mean that it is enough to verify that for each $t \in \mathrm{T}$ there is an $\mathrm{ACT}$ such that $\mathrm{A}$ is a relative neighborhood of $t$ and $E^{\prime}(\mathrm{A}) \subset E(\mathrm{~T})$. Therefore in discussing spectral synthesis one can restrict his attention to functions with compact spectrum. The immediate program is to relate spectral synthesis to another problem which is of considerable importance in the theory of approximation.

We denote by $V(\hat{\Gamma})$ the set of functions $\lambda=\hat{\mu}$, where $\mu \in B(\Gamma)$, with the norm $V(\lambda)=\|\mu\|$. We shall use the convention $V(\lambda)=\infty$ when $\lambda \notin V(\hat{\Gamma})$. Now given any $\mathrm{A} \subset \hat{\Gamma}$ and a continuous function $\lambda$ defined on $\mathrm{A}$ set $V_{\mathbf{A}}(\lambda)=\inf V(\tilde{\lambda})$ where the infimum is taken over all extrapolations $\tilde{\lambda}$ of $\lambda$, i.e. functions such that $\bar{\lambda}(t)=\lambda(t)$ for $t \in \mathbf{A}$; evidently $V_{\mathbf{A}}(\lambda)=\inf \|\mu\|$ where $\mu \in B(\Gamma)$ and $\lambda(t)$ $=\hat{\mu}(t)$ for $t \in \mathrm{A}$. $V(\mathrm{~A})$ denotes the set of $\lambda$ 's for which $V_{\mathbf{A}}(\lambda)<\infty$. Since $\lambda \in V(\mathbf{A})$ for any $\mathbf{A}$ implies that $\lambda$ is uniformly continuous on $\mathbf{A}$ and only continuous $\lambda$ 's come under consideration, there is no effect on $V(\mathrm{~A})$ or $V_{\mathbf{A}}$ when $\mathrm{A}$ is replaced by its closure $\overline{\mathrm{A}}$. Given $\lambda \in V(\mathrm{~A})$, a strictly continuous linear functional $L$ is defined on $E(\mathrm{~A})$ by $L(\varphi)=(\varphi, \mu)$ where $\mu \in B(\Gamma)$ and $\hat{\mu}(t)=\lambda(t)$ for $t \in \mathrm{A} ; L$ is independent of the choice of $\mu$ for the extrapolation. Considering $L$ as a bounded linear functional, one has obviously $\|L\| \leqq V_{\mathbf{A}}(\lambda)$. It is not denied that equality always holds, but we are able to prove this only in two situations.

4.1. Theorem. Suppose $\lambda \in V(\mathrm{~A})$ and let $L$ be the functional defined by $\lambda$ on $E(\mathrm{~A})$.

(i) If $\mathrm{A}$ is compact there exists a $\varphi \in E(\mathrm{~A})$ with $\|\varphi\|=1$ such that $L(\varphi)$ $=V_{\mathbf{A}}(\lambda)$.

${ }^{\left({ }^{30}\right)}$ Added May, 1959. Malliavin has shown that spectral synthesis is false in general for noncompact groups. 
(ii) If $\mathrm{A}$ is open, or more generally if for each $t \in \mathrm{A}$ there is $a \psi \in C_{0} \cap E(\mathrm{~A})$ such that $t \in \Lambda(\psi)$, there exists a $\mu \in B(\Gamma)$ with $\|\mu\|=\|L\|$ such that $\lambda(t)=\hat{\mu}(t)$ for $t \in A$.

Proof of (i). Since $\mathbf{A}$ is compact, given $\epsilon>0$ there is a $k \in L^{1}(\Gamma)$ with $\|k\|_{1}<1+\epsilon$ and $\hat{k}=1$ on A. From this observation it follows that $V_{\mathbf{A}}(\lambda)$ $=$ inf $\|f\|_{1}$ where the infimum is taken over all $f \in L^{1}(\Gamma)$ with $\hat{f}(t)=\lambda(t)$ for $t \in A$. In other words, given one such extrapolation $\hat{f}, V_{\mathbf{A}}(\lambda)=\inf \|f-g\|_{1}$ where the infimum is taken over all $g$ in the ideal $I_{\mathbf{A}}$ consisting of those functions in $L^{1}(\Gamma)$ whose Fourier transforms vanish on $\mathrm{A}$. Thus $\lambda$ determines an element, $l$, of the Banach algebra $L^{1}(\Gamma) / I(\mathrm{~A})$ and $V_{\mathrm{A}}(\lambda)=\|l\|$. The adjoint space of $L^{1}(\Gamma) / I(\mathrm{~A})$ is the annihilator subspace of $I(\mathrm{~A})$ in $L^{\infty}(\Gamma)$, i.e. $E(\mathrm{~A})$. Hence there is a $\varphi \in E(\mathrm{~A})$ with $\|\varphi\|=1$ such that $\|l\|=(l, \varphi)=L(\varphi)$.

Proof of (ii). By the Banach extension theorem, the restriction of $L$ to $C_{0} \cap E(\mathrm{~A})$ has a norm-preserving extension to all of $C_{0}(\Gamma)$. Thus, by the Riesz representation theorem, there exists a $\mu \in B(\Gamma)$ with $\|\mu\| \leqq\|L\|$ such that $L(\psi)=(\psi, \mu)$ for each $\psi \in C_{0} \cap E(\mathrm{~A}) . L$ being strictly continuous, it follows that if $t \in \Lambda(\psi)$ then $\hat{\mu}(t)=\left(e_{t}, \mu\right)=L\left(e_{t}\right)=\lambda(t)$.

For a fixed $\lambda, V_{\mathrm{T}}(\lambda)$ may be considered as a function of compact subsets $\mathbf{T}$ of $\hat{\Gamma}$. It is an increasing, non-negative function, but no other desirable properties are evident. In particular, as we shall soon see, the right-continuity of $V_{\mathrm{T}}(\lambda)$ is closely related to the spectral synthesis problem. To investigate this, let $\mathrm{T}$ be a given closed set, $\lambda$ a continuous function defined on a neighborhood of T, and set $V_{\mathrm{T}}^{\prime}(\lambda)=\inf V_{\mathbf{A}}(\lambda)$ where the infimum is taken over all $\mathbf{A}$ with $\mathrm{T} \subset$ Int $\mathrm{A}$.

4.2. Theorem. Suppose $\mathrm{T}$ is compact and $\lambda \in V(\mathrm{~T}+\mathrm{N})$ for some nucleus $\mathrm{N}$; let $L$ be the linear functional defined by $\lambda$ on $E^{\prime}(\mathrm{T})$. Then there exists a $\varphi \in E^{\prime}(\mathrm{T})$ with $\|\varphi\|=1$ such that $L(\varphi)=V_{\mathrm{T}}^{\prime}(\lambda)$. Hence a necessary and sufficient condition that $\mathrm{T}$ be an $S$-set is that $V_{\mathrm{A}}(\lambda)$ is right continuous at $\mathrm{T}$ for each $\lambda \in V(\Gamma)$.

Proof. The set-up is much the same as in (4.1)(i). $\lambda$ determines an element, $l$, of the Banach algebra $L^{1}(\Gamma) / I^{\prime}(\mathrm{T})$ where $I^{\prime}(\mathrm{T})$ is the smallest closed ideal in $L^{1}(\Gamma)$ containing the functions where Fourier transforms vanish on a neighborhood of T. The norm is $\|l\|=V_{\mathrm{T}}^{\prime}(\lambda)$; the adjoint space of $L^{1}(\Gamma) / I^{\prime}(\mathrm{T})$ is $E^{\prime}(\mathrm{T})$ and $(l, \varphi)=L(\varphi)$.

In view of the preceding theorem it is desirable to have estimates of $V_{\mathrm{T}}(\lambda)$ for given compact sets $\mathrm{T}$ and functions $\lambda \in V(\mathrm{~T})$. Some information can be obtained from the result: $\lim _{n \rightarrow \infty} V_{\mathrm{T}}^{\prime 1 / n}\left(\lambda^{n}\right)=\sup _{t \in \mathrm{T}}|\lambda(t)|$, which is standard functional analysis. (This also holds for noncompact $\mathrm{T}$ if one assumes $\lambda=\hat{\mu}$ where $\mu \in B(\Gamma)$ is the sum of an absolutely continuous measure and a discrete measure.) It is worthwhile recording a stronger statement which has applications to spectral analysis.

4.3. Theorem. Suppose $\mu \in B(\Gamma)$ and let $\mu_{n}$ be the $n$-fold convolution of $\mu$ 
with itself. Then if $\varphi \in C(\Gamma)$ has compact spectrum, lim $\sup _{n \rightarrow \infty}\left\|\mu_{n} \circ \varphi\right\|^{1 / n}$ $=\max |\hat{\mu}(t)|$ where the maximum is taken over $t \in \Lambda(\varphi)$.

The proof can be found in Domar, cf. Theorems 3.32 and 3.33.

This theorem has the particular consequence that if $\mathrm{T}$ is compact, $\lambda \in V(\mathrm{~T}+\mathrm{N})$ for some nucleus $\mathrm{N}$, and $\lambda=0$ on $\mathrm{T}$, then $\lim _{n \rightarrow \infty} V^{\prime 1 / n}\left(\lambda^{n}\right)=0$. One can show that if $\lambda$ satisfies a Lipschitz condition in a neighborhood of $\mathrm{T}$ then actually $V^{\prime}\left(\lambda^{n}\right)=0$ for sufficiently large $n$. This is the content of the next lemma in which the estimates are sharp.

4.4. Lemma ( $\left.{ }^{31}\right)$. Let $\mathrm{T}$ be a closed subset of $\hat{\Gamma}$ and $\mathrm{N}$ a nucleus. Suppose that $\lambda \in V(\mathrm{~T}+\mathrm{N})$ and that for some $J$-net $\{\mathrm{H}\},|\lambda(t)|=O\left(h^{1 / 2}\right)$ for $t \in \mathrm{T}+\mathrm{H}^{0}$. Then $V_{\mathbf{T}}^{\prime}(\lambda)=0$.

Proof. It suffices to establish the assertion for compact T. Let $\mu \in B(\Gamma)$ be such that $\hat{\mu}=\lambda$ on $\mathrm{T}+\mathrm{N}$, and consider $\varphi \in E^{\prime}(\mathrm{T})$. We have $\mu \circ \varphi$ $=\lim _{\mathrm{H} \rightarrow 0} \mu \circ\left(k_{\mathrm{H}} \varphi\right)$. Since $\mu \circ\left(k_{\mathrm{H}} \varphi\right)$ is the Fourier transform of $\lambda \Phi_{\mathrm{H}}$ when $\mathrm{H}^{0} \subset \mathrm{N}, \mu \circ \varphi=0$ will be a consequence of the fact that $\left\|\lambda \Phi_{\mathrm{H}}\right\|_{1}=o(1)$ as $\mathrm{H} \rightarrow 0$. This we now show. Let $\mathrm{T}_{\mathrm{H}}=\left(\mathrm{T}+\mathrm{H}^{0}\right) \cap \tilde{\mathrm{T}} . \lambda \Phi_{\mathrm{H}}$ vanishes outside $\mathrm{T}_{\mathrm{H}}$; the Schwarz inequality gives

$$
\begin{aligned}
\left\|\lambda \Phi_{\mathrm{H}}\right\|_{1} & =\int_{\mathrm{T}_{\mathrm{H}}}\left|\lambda(t) \Phi_{\mathrm{H}}(t)\right| d t \leqq\left\{\int_{\mathrm{T}_{\mathrm{H}}}|\lambda(t)|^{2} d t\right\}^{1 / 2}\left\{\int\left|\Phi_{\mathrm{H}}(t)\right|^{2} d t\right\}^{1 / 2} \\
& =O\left(h^{1 / 2}\right)\left|\mathrm{T}_{\mathrm{H}}\right|^{1 / 2} \cdot O\left(h^{-1 / 2}\right)=O\left(\left|\mathrm{~T}_{\mathrm{H}}\right|^{1 / 2}\right)=o(1)
\end{aligned}
$$

because, $\mathrm{T}$ being compact, $\left|\mathrm{T}_{\mathrm{H}}\right| \rightarrow 0$ as $\mathrm{H} \rightarrow 0$.

Obviously the same conclusion would hold had we assumed $|\lambda(t)|$ $=o\left(h^{1 / 2}\left|\mathrm{~T}_{\mathrm{H}}\right|^{-1 / 2}\right)$ for $t \in \mathrm{T}+\mathrm{H}^{0}$. This result is best possible in general as will be seen in an example at the end of the section, and the same idea can be extended to prove that the exponent $1 / 2$ in the lemma cannot be lowered.

So far we have only considered spectral synthesis in the strict topology. The difficulty of this problem suggests that we ask the more modest question of whether spectral synthesis holds in some weaker topology. Weakening the topology of $C(\Gamma)$ is essentially equivalent to replacing the full set of continuous linear functionals by a subspace of $B(\Gamma)$ consisting of measures satisfying a growth condition. This growth condition may be translated into a smoothness condition on the Fourier transforms. It will be seen soon that Lemma (4.4) is the key to a completely satisfactory solution.

4.5. THEOREM. Let $\Gamma$ be a group of rank $r$. If $r \leqq 2$ there is a locally convextopology on $C(\Gamma)$ which is weaker than the strict topology and stronger than the topology of uniform convergence on compact sets such that spectral analysis and

${ }^{(31)}$ The result is due to Beurling (unpublished). 
spectral synthesis hold. If $r \geqq 3$ spectral synthesis fails even for the topology of uniform convergence on compact sets.

REMARKs. The full proof of this theorem will be deferred until the end of $\$ 5$. Here we shall simply give the ideas and avoid technical complications. The example of Schwartz, given for $\Gamma=R^{3}$ shows that spectral synthesis does not even hold locally, i.e. in the topology of uniform convergence on compact sets (cf. the example at the end of this section). This result can be extended to general groups of rank $r \geqq 3$. In the other direction suppose $\Gamma=I^{r}$ or $R^{r}$ where $r \leqq 2$ and put $\delta(x)=|x|^{r / 2}$. The topology in question is given by the norm $\||\varphi|\|=\sup |\varphi(x) / 1+\delta(x)|$. The continuous linear functionals are given by those $\mu \in B(\Gamma)$ for which $\int \delta(x)|\mu(d x)|<\infty$. In this case $\hat{\mu}$ satisfies a suitable Lipschitz condition for the application of (4.4), and spectral synthesis follows immediately. More generally, if $\Gamma$ is compactly generated of rank $\leqq 2$, one can construct a pseudometric $\delta$ such that for each fixed $x \in \Gamma, \delta(n x)=O\left(n^{r / 2}\right)$ as $n \rightarrow \infty$, and one defines the norm in the same way with the same results. If $\Gamma$ is not compactly generated, one constructs a $\delta$ as above for each compactly generated open subgroup. The topology is then given by a set of pseudonorms. Finally one proves that the topology obtained is strong enough so that spectral analysis is preserved-the topology of uniform convergence on compact sets is too weak for spectral analysis when $r>0$.

A result of a different sort can be obtained from the spectral synthesis consequences of (3.6). Beurling $\left(^{32}\right)$ has shown that when $\Gamma=I^{1}$ or $R^{1}$ there are regular Banach algebras, which are subsets of $L^{1}(\Gamma)$ having $\hat{\Gamma}$ for the space of maximal ideals, for which the ideal theorem holds. The method is essentially restricted to groups of rank $\leqq 1$. For simplicity we shall confine ourselves here to the special case of groups of the form $\Gamma=\bigcup_{n=1}^{\infty} K_{n}$ where each $\mathrm{K}_{n}$ is a compact open subgroup of $\Gamma$. For $x \in \Gamma$ we write $|x|=\inf \left|\mathrm{K}_{n}\right|$ where the infimum is taken over those subgroups $\mathrm{K}_{n}$ containing $x$. One deduces from (4.4) that spectral synthesis holds in $C(\Gamma)$ in the topology given by $\|\varphi\| \|$ $=\sup _{x}|\varphi(x)| /\left(1+|x|^{1 / 2}\right)$, but $C(\Gamma)$ is not complete in this topology and we do not know that spectral synthesis is valid in the completed space. Stated in slightly different terms, the ideal theorem is not established for the algebra of functions $f \in L^{1}(\Gamma)$ with $\int|f(x)|\left(1+|x|^{1 / 2}\right) d x<\infty$. One can, however, obtain a closely related result. Let $\gamma(\xi)$ be a positive decreasing function defined for $\xi \geqq 0$ such that $\int \gamma(|x|) d x=1$. Define $A^{2}(\Gamma)$ to be the set of functions $f \in L^{1}(\Gamma)$ such that $\int|f(x)|^{2} \gamma^{-1}(|x|) d x<\infty$, for some such $\gamma$. Under the algebraic operations inherited from $L^{1}(\Gamma)$ and the norm $N(f)$ $=\inf _{\gamma}\left\{\int|f(x)|^{2} \gamma^{-1}(|x|) d x\right\}^{1 / 2}, A^{2}(\Gamma)$ is a regular commutative Banach algebra with involution whose maximal ideal space is $\hat{\mathrm{r}}$. We omit the verifica-

(32) See Analysis in some convolution algebras in SyMPOSIUM. 
tion( $\left.{ }^{33}\right)$. The dual space of $A^{2}(\Gamma)$ is $B^{2}(\Gamma)$ consisting of those measurable functions $\varphi$ for which $\int|\varphi(x)|{ }^{2} \gamma(|x|) d x<\infty$ for each admissible $\gamma$. Now we shall show that the ideal theorem holds in $A^{2}(\Gamma)$. Let $J$ be a closed ideal of $A^{2}(\Gamma)$ and set $\mathbf{T}=\bigcap_{o \in J} \mathbf{Z}(g)$. Suppose $f \in A^{2}(\Gamma)$ and $\mathbf{T} \subset \mathbf{Z}(f)$; we wish to show that $f \in J$. To do this it is sufficient to demonstrate that if $\varphi \in B^{2}(\Gamma)$ and $(g, \varphi)=0$ for each $g \in J$ then $(f, \varphi)=0$. Choose some admissible $\gamma$ so that $\int|f(x)|{ }^{2} \gamma^{-1}(|x|) d x<\infty$. Let $q(x)=\gamma(|x|)$. Then $\varphi \in L_{q}^{2}$. Using the notation of $\S 3$, we also have that $\varphi \in \bar{Q}_{\mathrm{T}}$. Now if we write $q(0)=\epsilon^{-1}, \nu(x)=q^{-1}(x)$ $-q^{-1}(0)$ we have $q(x)=[\epsilon+\nu(x)]^{-1}$ where $\nu$ is an ultrametric on $\Gamma$ and is hence negative definite. Using (3.6) one sees that $(f, \varphi)=0$ for every $\varphi \in \bar{Q}_{\mathbf{T}}$.

The cases $\Gamma=I^{1}$ and $\Gamma=R^{1}$ are treated in a similar fashion. The above discussion is applicable with $|x|$ having the ordinary meaning. Where the essential complicaton arises is that one can no longer take $q(x)=\gamma(|x|)$; the details of the appropriate modifications can be gathered from BEURLING [2]. In summary, we have

4.6. TheOREM. Suppose that $\Gamma$ is the countable union of compact subgroups or that $\Gamma=I^{1}$ or $R^{1}$. Then there is a subset, $A^{2}(\Gamma)$, of $L^{1}(\Gamma)$ which forms a regular commutative Banach algebra with involution having $\hat{\Gamma}$ as the space of regular maximal ideals such that the ideal theorem holds in $A^{2}(\Gamma)$.

ExAmple. Suppose $\Gamma=R^{3}$ and $\Sigma \subset \hat{\Gamma}$ a closed surface of class $C^{(3)}$ with everywhere strictly positive curvature. Let $\alpha$ be the distribution of surface area on $\Sigma$, and set $\varphi(x)=\int e(t x) \alpha(d t)$. It can be proved that $|x| \cdot|\varphi(x)|$ is bounded. Now suppose $\mu \in B(\Gamma)$ and $\int\left|x_{1}\right||\mu(d x)|<\infty$, with $\hat{\mu}=0$ on $\Sigma$. If we write $\psi(x)=x_{1} \varphi(x)$ then $\Lambda(\psi) \subset \Sigma$ and $\mu \circ \psi(x)=-\int \varphi(x-y) y_{1} \mu(d y)$. Thus $\mu \circ \psi$ cannot be identically zero unless $\partial \hat{\mu} / \partial t_{1}$ vanishes on $\Sigma$. Since one can always find an appropriate $\mu, \Sigma$ is not an $S$-set. The first example of this type is that of Schwartz. For another example let $\Sigma$ be the surface $\sin ^{2} t_{1} / 2$ $+\sin ^{2} t_{2} / 2+\sin ^{2} t_{3} / 2=c$ with $0<c<1 / 2$ where we take only the component with $\left|t_{j}\right|<\pi, j=1,2,3$. One can choose an appropriate $\mu$ which is a measure concentrated at a finite set of points, namely the one with $\hat{\mu}(t)=3-2 c$ $-\cos t_{1}-\cos t_{2}-\cos t_{3}$. This example shows that spectral synthesis does not hold in $I^{3}$ even in the topology of convergence on finite sets. It is also worth noticing that the general example indicates the sharpness of the estimate in the remark following (4.4). We have $\left|\Sigma_{\mathrm{H}}\right|=O\left(h^{+1 / 3}\right)$ so that the term $O\left(h^{-1 / 2}\left|\Sigma_{\mathrm{H}}\right|^{+1 / 2}\right)$ is $O\left(h^{-1 / 3}\right)$. In our examples with $\lambda(t)=\hat{\mu}(t)$ we may have $\hat{\mu}(t)=O\left(h^{1 / 3}\right)$ for $t \in \Sigma+H^{0}$, but $\hat{\mu}(t)=o\left(h^{1 / 3}\right)$ implies that the first partial derivatives of $\hat{\mu}$ vanish on $\Sigma$.

5. Representations. As noted in the introduction, given a continuous map $\rho: \Gamma \rightarrow \mathrm{P}$ of one locally compact Hausdorff space into another, there always arises a linear map $\hat{\rho}: C(\mathrm{P}) \rightarrow C(\Gamma)$ which is continuous in both the norm and

(33) It is not entirely trivial, but it is substantially the same as that given in the preceding reference to Beurling. 
the strict topologies. On account of the continuity of $\hat{\rho}$ in the strict topology, there is a dual map which we also denote by $\rho$, namely $\rho: B(\Gamma) \rightarrow B(\mathrm{P})$; this map is continuous in the norm topology. A closer analysis follows. Let $\mathcal{F}$ be the smallest $\sigma$-ring of subsets of $\mathbf{P}$ containing the images under $\rho$ of compact subsets of $\Gamma$. $\mathcal{F}$ consists entirely of Borel sets of $P$ and every Borel set which is a subset of a member of $\mathcal{F}$ itself belongs to $\mathcal{F}$. Given $\nu \in B(\mathrm{P})$ there is a unique decomposition $\nu=\nu_{0}+\nu^{\prime}$ where $\nu_{0}$ is concentrated on $\mathcal{F}$ and each set of $\mathcal{F}$ is a null set for $\nu^{\prime}$. The decomposition is obtained this way. Consider $\sup _{B \in \mathcal{F}}|\nu|(B)$; because of the complete additivity of $\nu$, the supremum is attained for some $\mathbf{B}_{0}$ in the $\sigma$-ring $\mathcal{F}$. Now for any Borel subset $\mathbf{B}$ of $\mathbf{P}$ set $\nu_{0}(B)=\nu\left(B \cap B_{0}\right), \nu^{\prime}(B)=\nu\left(B \cap \tilde{B}_{0}\right)$. Note that $\|\nu\|=\left\|\nu_{0}\right\|+\left\|\nu^{\prime}\right\|$. Thus we have a direct sum decomposition $B(\mathrm{P})=B_{0} \oplus B^{\prime}$. It is clear that $\rho B(\Gamma) \subset B_{0}$; we wish to show further that given $\nu_{0} \in B_{0}$ there exists a $\mu \in B(\Gamma)$ with $\|\mu\|=\left\|\nu_{0}\right\|$ such that $\rho \mu=\nu_{0}$. To do this we observe that given a set $\mathbf{B}_{0} \in \mathcal{F}$ supporting $\nu_{0}$ we can find a sequence $\left\{K_{i}\right\}$ of compact subsets of $\Gamma$ such that $B_{0} \subset \bigcup_{i=1}^{\infty} \rho K_{i}$. Replace $\nu_{0}$ by its restriction to $\bigcup_{i=1}^{n} \rho \mathbf{K}_{i}$. If we can show that whenever $K$ is a compact subset of $\Gamma$ and $\nu_{0} \in B(\mathbf{P})$ with $\Sigma\left(\nu_{0}\right) \subset \rho \mathrm{K}$ there is a $\mu \in B(\boldsymbol{\Gamma})$ with $\Sigma(\mu) \subset \mathrm{K},\|\mu\|=\left\|\nu_{0}\right\|$, and $\rho \mu=\nu_{0}$ the assertion will follow for arbitrary $\nu_{0} \in B_{0}$ by a limiting argument. However $\hat{\rho}: C(\rho \mathbf{K}) \rightarrow C(\mathrm{~K})$ is norm-preserving and, since $\mathbf{K}$ and $\rho \mathbf{K}$ are compact, the adjoint map of the dual Banach spaces is $\rho: B(\mathrm{~K}) \rightarrow B(\rho \mathrm{K})$. In this situation the isometry of $\hat{\rho}$ yields the required properties of $\rho$. To summarize what has been done we state

5.1. Theorem. Given two locally compact Hausdorff spaces $\Gamma$ and $\mathrm{P}$ and a continuous map $\rho: \Gamma \rightarrow \mathrm{P}$ there is induced a linear transformation $\rho: B(\Gamma)$ $\rightarrow B(\mathrm{P})$ and a direct sum decomposition $B(\mathrm{P})=\rho B(\Gamma) \oplus B_{\rho}^{\prime}$, meaning that each $\nu \in B(\mathbf{P})$ may be written in the form $\nu=\rho \mu+\nu^{\prime}$ where $\mu \in B(\Gamma), \nu^{\prime}(\rho \mathrm{K})=0$ for each compact $\mathrm{K} \subset \Gamma$, and $\|\nu\|=\|\mu\|+\left\|\nu^{\prime}\right\|$.

The statement of the theorem is not intended to suggest that $\mu$ is uniquely determined by $\nu$; this may be false if $\rho$ is not one-to-one.

The foregoing discussion serves as a preliminary to investigation of the situation in which $\Gamma$ and $P$ are groups and $\rho$ is a representation. In this case the restriction of $\hat{\rho}: C(\mathrm{P}) \rightarrow C(\Gamma)$ to the characters gives a representation $\hat{\rho}: \hat{\mathbf{P}} \rightarrow \hat{\Gamma}$. The notation will be further abused by writing $\rho: C(\hat{\Gamma}) \rightarrow C(\hat{\mathrm{P}})$ and $\hat{\rho}: B(\hat{\mathrm{P}}) \rightarrow B(\hat{\Gamma})$ for the induced maps. What is new under the additional hypotheses is that $\rho: B(\Gamma) \rightarrow B(\mathrm{P})$ is a homomorphism of the Banach algebras and the ring $\mathcal{F}$ described above is closed under group-theoretic addition of sets. From the second fact it follows that if $\mu \in B(\Gamma)$ and $\nu^{\prime} \in B_{\rho}^{\prime}$ then $\rho \mu \circ \nu^{\prime} \in B_{\rho}^{\prime}$ for if $\mathrm{B} \in \mathcal{F}$ then $\rho \mu \circ \nu^{\prime}(\mathrm{B})=\int_{\mathrm{P}} \nu^{\prime}(\mathrm{B}-r) \rho \mu(d r)=\int_{\hat{\Gamma}} \nu^{\prime}(\mathrm{B}-\rho x) \mu(d x)$ $=0$ since $\mathbf{B}-\rho x \in \mathcal{F}$ whenever $\mathbf{B} \in \mathcal{F}$. The corresponding revision of (5.1) is

5.1'. Theorem. Given two groups $\Gamma$ and $\mathrm{P}$ and a representation $\rho: \Gamma \rightarrow \mathrm{P}$ there is induced a homomorphism $\rho: B(\Gamma) \rightarrow B(\mathrm{P})$ and a direct sum decomposition 
$B(\mathrm{P})=\rho B(\Gamma) \oplus B_{\rho}^{\prime}$ as in (5.1) with the additional statement that $\rho B(\Gamma) \circ B_{\rho}^{\prime}$ $=B_{\rho}^{\prime}$.

The question which has important consequences for spectral theory is the characterization of $\rho B(\Gamma)$ as a sub-ring of $B(\mathrm{P})$ in terms of Fourier transforms. It is clear that $\nu=\rho \mu$ if and only if $\hat{\nu}=\rho \hat{\mu}$. A nontrivial reformulation of the condition is

5.2. Theorem. Given a representation $\rho: \Gamma \rightarrow P$ and $\nu \in B(\mathrm{P})$, a necessary and sufficient condition that $\nu=\rho \mu$ for some $\mu \in B(\Gamma)$ is that $\hat{\nu}=\rho \lambda$ for some $\lambda \in C(\hat{\Gamma})$.

Proof. Only the sufficiency is nonobvious, and it will suffice to establish it in each of the two cases: (i) $\rho$ is a homomorphism onto and (ii) $\rho$ is faithful; the general result follows from composing the two special cases. In (i) what has to be proved is that $B(\mathrm{P})=\rho B(\Gamma)$. This is true because, when $\rho$ is a homomorphism onto, each compact subset of $\mathrm{P}$ is of the form $\rho \mathrm{K}$ where $\mathrm{K}$ is a compact subset of $\Gamma$. This implies that $\mathcal{F}$ consists of all the Borel subsets of $\mathrm{P}$, and that does it. Case (ii) offers more difficulty; the proof is modelled after that of Bochner [1] where the theorem is proved for $\Gamma$ the real line and $\mathrm{P}$ its Bohr compactification. One starts with the extension to groups of a modification of Bochner's result given by SCHOENBERG, namely that a necessary and sufficient condition for $\theta \in C(\hat{\mathrm{P}})$ to have $V(\theta) \leqq M$ is that $\left|\int \theta(u) \hat{g}(u) d u\right| \leqq M\|\hat{g}\|$ for each $\hat{g} \in L^{1}(\hat{\mathrm{P}})$. (Schoenberg's proof extends to groups mutatis mutandis.) One has immediately that a necessary and sufficient condition for $\lambda \in C(\Gamma)$ to have $V(\rho \lambda) \leqq M$ is that $\left|\int \lambda(\hat{\rho} u) \hat{g}(u) d u\right| \leqq M\|g\|$ for each $\hat{g} \in L^{1}(\hat{\mathrm{P}})$. It is also necessary that for each $v \in \hat{\mathrm{P}},|\lambda \lambda(\hat{\rho} u-\hat{\rho} v) \hat{g}(u) d u|$ $\leqq M\|g\|$. $\rho$ being one-to-one, $\hat{\rho} \hat{\mathrm{P}}$ is dense in $\hat{\Gamma} \cdot \int \lambda(\hat{\rho} u-t) \hat{g}(u) d u$ is continuous; therefore it is necessary that $\left|\int \lambda(\hat{\rho} u-t) \hat{g}(u) d u\right| \leqq M\|g\|$. Using this strong form of the necessity together with the weak form of the sufficiency, we see that if $\lambda \in C(\hat{\Gamma}), V(\rho \lambda) \leqq M$, and $\alpha \in B(\hat{\Gamma})$ then $V(\rho(\alpha \circ \lambda)) \leqq M\|\alpha\|$. Now we prove the theorem under the extra hypothesis that $\lambda \in L^{1}(\hat{\Gamma})$. It is assumed that $\rho \lambda=\hat{\nu}$ where $\nu \in B(\mathrm{P})$. By the preceding analysis, $\rho\left(\hat{k}_{\mathrm{H}} \circ \lambda\right)=\hat{\nu}_{\mathrm{H}}$ where $\hat{\nu}_{\mathrm{H}} \in B(\mathrm{P})$ and $\left\|\nu_{\mathrm{H}}\right\| \leqq\|\nu\|$. The extra hypothesis guarantees that $\hat{k}_{\mathrm{H}} \circ \lambda=\hat{\mu}_{\mathrm{H}}$ where $\mu_{\mathrm{H}} \in B(\Gamma)$. Hence $\nu_{\mathrm{H}}=\rho \mu_{\mathrm{H}}$ and $\left\|\mu_{\mathrm{H}}\right\| \leqq\|\nu\|$. The last inequality establishes the conditional $w^{*}$-compactness of the collection $\left\{\mu_{\mathrm{H}}\right\}$. It is easy to see that $\lim _{\mathrm{H} \rightarrow 0} \mu_{\mathrm{H}}=\mu$ exists in the $w^{*}$-topology of $B(\Gamma)$ and that $\lambda=\hat{\mu}$. The superfluous assumption, $\lambda \in L^{1}(\hat{\Gamma})$, is readily removed-this we leave to the reader.

$\left(5.1^{\prime}\right)$ and (5.2) combined lead to a result which yields a number of important consequences.

5.3. THEOREM. Let $\rho: \Gamma \rightarrow P$ be a representation and $\Omega$ an open subset of $\hat{\Gamma}$ such that $\hat{\rho} \hat{\mathrm{P}} \cap \Omega$ is dense in $\Omega$. Then $\rho$ maps $V(\Omega)$ isometrically into $V\left(\hat{\rho}^{-1} \Omega\right)$ and $\rho V(\Omega)=V\left(\hat{\rho}^{-1} \Omega\right) \cap \rho C(\Omega)$. 
Proof. It is trivial that $\rho$ is a norm-diminishing map of $V(\Omega)$ into $V\left(\hat{\rho}^{-1} \Omega\right)$ whose image is contained in $V\left(\hat{\rho}^{-1} \Omega\right) \cap \rho C(\Omega)$. What we have to prove is that if $\lambda \in C(\Omega)$ and there is a $\nu \in B(\mathrm{P})$ such that $\lambda(\hat{\rho} u)=\hat{\nu}(u)$ whenever $\hat{\rho} u \in \Omega$, then there exists a $\mu \in B(\Gamma)$ with $\|\mu\| \leqq\|\nu\|$ such that $\lambda(t)=\hat{\mu}(t)$ for $t \in \Omega$. Let $\mu$ be chosen as in the decomposition $\nu=\rho \mu+\nu^{\prime}$ given by $\left(5.1^{\prime}\right)$. Certainly $\|\mu\| \leqq\|\nu\|$ so that if we prove $\hat{\nu}^{\prime}(u)=0$ for $u \in \hat{\rho}^{-1} \Omega$ we are through for then $\lambda(t)=\hat{\mu}(t)$ whenever $t \in \hat{\rho} \hat{\mathrm{P}} \cap \Omega$ and hence for all $t \in \Omega$ since this subset is dense. Consider a point $u \in \rho^{-1} \Omega$. $\Omega$ being open, we can find $\kappa \in B(\Gamma)$ such that $\hat{\kappa}(\hat{\rho} u) \neq 0$ while $\hat{\kappa}=0$ outside $\Omega$. The Fourier-Stieltjes transform of $\rho \kappa \circ \nu$ is equal to $\rho(\hat{\kappa} \lambda)$ where $\hat{\kappa} \lambda$ is defined to be 0 on the complement of $\Omega$. According to (5.2) $\rho \kappa \circ \nu \in \rho B(\Gamma)$. However $\rho \kappa \circ \nu^{\prime} \in B_{\rho}^{\prime}$; hence $\rho \kappa \circ \nu^{\prime}=0$. This means $\hat{\boldsymbol{\kappa}}(\hat{\rho} u) \hat{\nu}^{\prime}(u)=0$. We conclude that $\hat{\nu}^{\prime}$ is zero on $\hat{\rho}^{-1} \Omega$.

The first conclusion we wish to draw is this.

5.4. Corollary. Suppose the hypotheses of (5.3) are satisfied and that $\varphi \in E(\Omega), \epsilon>0$, and $\mathrm{K}$ a compact subset of $\Gamma$ are given. Then there exists a $\psi \in E\left(\hat{\rho}^{-1} \Omega\right)$ such that $\|\psi\| \leqq\|\varphi\|$ and $|\varphi(x)-\psi(\rho x)|<\epsilon$ for $x \in \mathrm{K}$.

Proof. Let $F$ be the set of functions $\psi \in E\left(\hat{\rho}^{-1} \Omega\right)$ with $\|\psi\| \leqq\|\varphi\|$. It suffices to prove that $\varphi$ is in the strict closure of $\rho F$. Since $\rho F$ is convex and circled, if this were not so there would exist a $\mu \in B(\Gamma)$ with $(\varphi, \mu)>\|\varphi\|$ such that $|(\psi, \mu)| \leqq\|\varphi\|$ for each $\psi \in F$. The last inequality is equivalent to $V_{\hat{\rho}-1 \Omega}(\rho \hat{\mu})$ $\leqq 1$. (5.3) asserts that then $V_{\Omega}(\hat{\mu}) \leqq 1$ contradicting the assumption $(\varphi, \mu)$ $>\|\varphi\|$.

It is of interest to restate (5.3) and (5.4) in a special case.

5.5. THEOREM. Let $\Omega$ be an open set in $\hat{\Gamma}$ and $\Omega_{0}$ a dense subset of $\Omega$ such that each integral linear combination of elements of $\Omega_{0}$ which lies in $\Omega$ is contained in $\Omega_{0}$, e.g. if $\Omega$ is an open subset of the real line one could take $\Omega_{0}$ to be the rational points of $\Omega$.

(i) A necessary and sufficient condition for $\lambda \in C(\Omega)$ to have the property $V_{\Omega}(\lambda) \leqq M$ is that

$$
\left|\sum c_{i} \lambda\left(t_{i}\right)\right| \leqq M \sup _{x \in \Gamma}\left|\sum c_{i} e\left(t_{i} x\right)\right|
$$

for each finite collection $\left\{\left(c_{i}, t_{i}\right)\right\}$ of pairs of complex numbers $c_{i}$ and points $t_{i} \in \Omega_{0}$.

(ii) Given $\varphi \in E(\Omega), \epsilon>0$, and $\mathrm{K}$ a compact subset of $\Gamma$ there is a trigonometric polynomial, $\psi(x)=\sum c_{i} e\left(t_{i} x\right)$ (a finite sum), with $t_{i} \in \Omega_{0}$ such that $\|\psi\| \leqq\|\varphi\|$ and $|\varphi(x)-\psi(c)|<\epsilon$ for $x \in \mathrm{K}$.

One has only to take $\hat{\mathbf{P}}$ to be the discrete group isomorphic to the abstract group generated by $\Omega_{0}$ in $\hat{\Gamma}$ and $\hat{\rho}: \hat{\mathrm{P}} \rightarrow \hat{\Gamma}$ the natural map to see that (i) is simply a version of (5.3) and that (ii) is a banal deduction from (5.4). Nevertheless, the statements are worth commenting upon. The theorem is essen- 
tially due to Krein $\left({ }^{34}\right)$, who considers only $\Gamma=R$, when $\Omega_{0}$ has the additional property that each finite collection of points in its generates a discrete subgroup of $\hat{\Gamma}$. The extra assumption leads to some loss of generality even in the classical case, e.g. $\hat{\Gamma}=R / I$ and $\Omega_{0}$ consists of those fractional parts $((n \vartheta))$ of the multiples of a fixed irrational $\vartheta$ which lie in $\Omega$. Also, the condition on $\Omega_{0}$ cannot be fulfilled for certain groups of which $\Gamma=$ rationals mod 1 in the discrete topology is an example. However Krein's method has the important advantage that it gives a prescription for the construction of the approximating polynomials. This matter will be taken up after (5.13). Another observation is that if $\Omega$ is open and $\varphi \in E(\Omega)$ the synthesis is possible in the narrow topology; this provides the strengthening of the theorem of Godement given earlier, $\left(1.3^{\prime}\right)$.

In passing we mention another corollary of (5.2). Suppose the group $\mathbf{P}$ is given and that $\Gamma$ is the same abstract group with the discrete topology, $\rho$ being the isomorphism of the abstract groups. One may identify $\rho C(\hat{\Gamma})$ with the uniformly almost periodic functions on $\hat{\mathrm{P}}$. Thus (5.2) in this case is the well-known assertion that $\hat{\nu}$ is almost periodic if and only if $\nu$ is a discrete measure.

At this point it is necessary to investigate the effect on the spectrum of the $\operatorname{map} \hat{\rho}: C(\mathrm{P}) \rightarrow C(\Gamma)$.

5.6. Lemma $\left.{ }^{35}\right)$. Let $\rho: \Gamma \rightarrow \mathrm{P}$ be a representation and suppose that $\mathrm{P}_{\rho}^{\prime}$ is a subset of $\mathbf{P}$ such that $\rho \Gamma+\mathbf{P}_{\rho}^{\prime}$ is dense in $\mathbf{P}$. Then for $\psi \in C(\mathbf{P})$, the closure of $\hat{\rho} \Lambda(\psi)$ is the smallest closed set in $\hat{\Gamma}$ containing the sets $\Lambda\left(\hat{\rho} \psi^{r}\right), r \in \mathrm{P}_{\rho}^{\prime}$.

Proof. If $\mu \in B(\Gamma)$ and $\hat{\mu}$ vanishes on a neighborhood of a $\hat{\rho} \Lambda(\psi)$ then $\rho \hat{\mu}$ vanishes on a neighborhood of $\Lambda(\psi)$. Therefore $\left(\psi^{r}, \rho \mu\right)=0$ for each $r \in P$, i.e. $\mu \circ \rho \psi^{r}=0$ for each $r$. This proves that $\Lambda\left(\hat{\rho} \psi^{r}\right)$ is included in the closure of $\hat{\rho} \Lambda(\psi)$. Conversely, if $\hat{\boldsymbol{\mu}}$ vanishes on an open set containing each $\Lambda\left(\hat{\rho} \psi^{r}\right)$ for $r \in \mathrm{P}_{\rho}^{\prime}$ then $\mu \circ \hat{\rho} \psi^{r}=0$. This is the same as saying $\rho \mu \circ \psi$ is zero at each point of $\rho \Gamma+\mathrm{P}_{\rho}^{\prime}$; the hypothesis that this set is dense implies that $\rho \mu \circ \psi=0$. We can conclude that $\hat{\rho} \Lambda(\psi)$ is contained in the closure of the union of the sets $\Lambda\left(\hat{\rho} \psi^{r}\right)$.

It is an immediate consequence of the above lemma that if $\mathrm{T}$ is a closed subset of $\hat{\Gamma}$ then $\rho$ induces a map $\hat{\rho}: E^{\prime}\left(\hat{\rho}^{-1} \mathrm{~T}\right) \rightarrow E^{\prime}(\mathrm{T})$; on account of the continuity of $\hat{\rho}$ in the strict topology we even have $\hat{\rho}: E\left(\hat{\rho}^{-1 T}\right) \rightarrow E(\mathrm{~T})$. If we assume that $\hat{\rho} \hat{\mathbf{P}} \supset \mathrm{T}$ then it is trivial that the image of $E\left(\hat{\boldsymbol{\rho}}^{-1} \mathrm{~T}\right)$ is dense in $E(\mathbf{T})$. The corresponding statement with $E$ replaced by $E^{\prime}$ cannot be true in general, for if we let $\mathrm{P}$ be the Bohr compactification of $\Gamma$ and $\rho$ the natural imbedding, $\hat{\rho} E^{\prime}\left(\hat{\rho}^{-1} \mathrm{~T}\right)$ dense in $E^{\prime}(\mathrm{T})$ implies that $\mathrm{T}$ is an $S$-set. However, if we restrict $\rho$ to be a homomorphism the statement is true. The substance of the work

(34) See Achieser, p. 146.

(36) This is due to REITER [2] in the case that $\rho$ is a homomorphism. 
of REITER [2] is the study of the case when $\rho$ is a homomorphism, and the important results are contained in the next two theorems which are essentially proved there.

5.7. TheOREM. Let $\rho: \Gamma \rightarrow \mathrm{P}$ be a homomorphism and $\mathrm{T}$ a closed subset of $\hat{\Gamma}$ such that $\hat{\rho} \hat{\mathrm{P}} \supset \mathrm{T}$. Given $\varphi \in E^{\prime}(\mathrm{T}), \epsilon>0$, and $\mathrm{K}$ a compact subset of $\Gamma$ there exists $a \psi \in E^{\prime}\left(\hat{\rho}^{-1} \mathrm{~T}\right)$ such that $\|\psi\| \leqq\|\varphi\|$ and $|\varphi(x)-\psi(\rho x)|<\epsilon$ for $x \in \mathrm{K}$.

The reader is invited to compare (5.4) and (5.7). The other theorem is sort of a converse to the statement that $\hat{\rho}$ maps $E\left(\hat{\rho}^{-1} \mathrm{~T}\right)$ into a dense subset of $E(\mathrm{~T})$.

5.8. THEOREM. Suppose the hypotheses of (5.7) are satisfied and $\psi \in C(\mathrm{P})$ is given. If $\hat{\rho} \psi^{r} \in E(\mathrm{~T})$ for each $r \in \mathrm{P}_{\rho}^{\prime}$, where $\rho \Gamma+\mathrm{P}_{\rho}^{\prime}$ is dense in $\mathrm{P}$, then $\psi \in E\left(\hat{\rho}^{-1} \mathrm{~T}\right)$.

Now (5.6) itself suggests a refinement; namely, if $\hat{\rho}$ is one-to-one on $\Lambda(\psi)$ we would like to have the closure of $\hat{\rho} \Lambda(\psi)$ equal to $\Lambda(\hat{\rho} \psi)$. This is not true in general even if $\rho$ is restricted to be a homomorphism. The situation is readily reduced to the matter of determining under what circumstances the hypotheses $\hat{\rho}$ one-to-one on $\Lambda(\psi)$ and $\hat{\rho} \psi=0$ lead to the conclusion that $\psi=0$. Statements of this nature are called uniqueness theorems, for what we are saying is that $\psi \in C(\mathrm{P})$ is uniquely determined by its values on $\rho \Gamma$. A strong form of a uniqueness theorem is an interpolation formula. In a natural way, uniqueness theorems are companions of approximation theorems such as (5.4) and (5.7). Our next aim is to investigate some cases where rather powerful uniqueness and approximation theorems hold. These are closely related to wellknown propositions in the theory of entire functions of exponential type, a subject which we shall discuss from the spectral theory viewpoint anon.

The general problem of uniqueness and approximation theorems is readily reduced to the situation where one studies a group and a subgroup. It will be convenient to change the notation here. We shall consider a group $\Gamma$ with a subgroup $\Delta$ and the injection $\iota: \Delta \rightarrow \Gamma . \Delta^{\perp}$ denotes the annihilator subgroup in $\hat{\Gamma}$ of $\Delta$. We shall occasionally abuse the notation by identifying the subgroups with their corresponding subsets. It will be convenient to have a construction which is used frequently in classical analysis and has been generalized to groups by Reiter; it forms the basis for much of his investigations; for proofs of the properties quoted below the reader is referred to his works. Let $\mathrm{T}$ be a compact subset of $\hat{\Gamma}$ and $N$ a nucleus. We set $l_{T, N}=|N|^{-1} \chi_{T+N} \bar{\chi}_{N}$. We have $l_{\mathrm{T}, \mathrm{N}} \in L^{1}(\Gamma), l_{\mathrm{T}, \mathrm{N}}$ vanishes outside $\mathrm{T}+\mathrm{N}^{0}$; and not only is $l_{\mathrm{T}, \mathrm{N}}=1$ on $\mathrm{T}$ but $V_{\mathrm{T}}^{\prime}\left(1-l_{\mathrm{T}, \mathrm{N}}\right)=0$. We also require a simple consequence of the Poisson summation formula $\left({ }^{36}\right)$, namely, suppose $\Gamma / \Delta$ is compact and that $\mathrm{H}$ is a Jordan measurable subset of $\hat{\Gamma}$ with compact closure such that $\mathrm{H}^{0} \cap \Delta^{\perp}=0$; then if $f \in L^{2}(\Gamma)$ and $\hat{f}=0$ almost everywhere outside $H$ one has $\int_{\Gamma}|f(x)|^{2} d x$ $=\int_{\Delta}|f(\iota y)|{ }^{2} d y$ where the Haar measures on $\Gamma$ and $\Delta$ are normalized to make

${ }^{(36)}$ Cf. Loomis, pp. 152-153. 
the induced Haar measure of the compact group $\Gamma / \Delta$ equal to unity. At this point we can prove

5.9. Theorem. Suppose $\Gamma / \Delta$ is compact and that $\mathrm{T}$ is a compact subset of $\hat{\Gamma}$ no two points of which are congruent modulo $\Delta^{\perp}$. Then the map $\hat{\imath}: E^{\prime}(\mathrm{T}) \rightarrow E^{\prime}(\hat{\imath} \mathrm{T})$ induced by the injection $\iota: \Delta \rightarrow \Gamma$ is an isomorphism of the two Banach spaces.

Proof. The hypotheses assert that $(\mathrm{T}-\mathrm{T}) \cap \Delta^{\perp}=0$, but $\Delta^{\perp}$ is discrete since $\Gamma / \Delta$ is compact. Therefore there is a nucleus $\mathrm{N}$ such that for the set $\mathrm{H}=\mathrm{T}+\mathrm{N}$ we have $\mathrm{H}^{0} \cap \Delta^{\perp}=0$. For a given $\varphi \in E^{\prime}(\mathrm{T})$ and $x \in \Gamma$ put $f_{1}(y)=\bar{\chi}_{\mathrm{H}}(x-y)$, $f_{2}(y)=|\mathrm{N}|^{-1} \chi_{\mathrm{N}}(y) \varphi(y)$. Then for $j=1,2$ we have $f_{j} \in L^{2}(\Gamma)$ and $\hat{f}_{j}=0$ almost everywhere outside $\mathrm{H}$. Thus $\int_{\Gamma}\left|f_{j}(z)\right|^{2} d z=\int_{\Delta}\left|f_{j}(\iota y)\right|^{2} d y$ which implies that $\int_{\mathrm{r}} \bar{f}_{1}(z) f_{2}(z) d z=\int_{\Delta} \bar{f}_{1}(\iota y) f_{2}(\iota y) d y$. Now $\bar{f}_{1}(y) f_{2}(y)=l_{\mathrm{T}, \mathrm{N}}(x-y) \varphi(y)$. Since $\varphi \in E^{\prime}(\mathrm{T})$, the integral on the left is simply $\varphi(x)$ and we have obtained the formula $\left({ }^{37}\right)$

$$
\varphi(x)=\int_{\Delta} l_{\mathrm{T}, \mathrm{N}}(x-\iota y) \varphi(\iota y) d y .
$$

This proves that $\hat{\imath}: E^{\prime}(\mathrm{T}) \rightarrow E^{\prime}(\hat{\imath} \mathrm{T})$ is one-to-one. To show that it is onto we employ the device already used together with the Schwarz inequality to prove that $\int_{\Delta}\left|l_{\mathrm{T}, \mathrm{N}}(x-\iota y)\right| d y \leqq|\mathrm{~N}|^{-1 / 2}|\mathrm{~T}+\mathrm{N}|^{1 / 2}$ and that the map from $\Gamma$ to $L^{1}(\Delta)$ given by $x \rightarrow l_{\mathrm{T}, \mathrm{N}}(x-\imath y)$ is continuous. Thus, given $\psi \in C(\Delta)$ we may define a function $\varphi \in C(\Gamma)$ by $\varphi(x)=\int_{\Delta} l_{\mathrm{T}, \mathrm{N}}(x-\iota y) \psi(y) d y$. Now if $\psi=\imath e_{t}$ where $t \in \mathrm{T}$ it is easy to see that $\varphi=e_{t}$. From this it follows by approximation that if $\psi \in E(\hat{\imath} T)$ then $\varphi \in E(T)$ and $\hat{\imath} \varphi=\psi$. In case we only know that $\psi \in E^{\prime}(T)$ we choose a set $\mathrm{A}$ containing $\mathrm{T}$ in its interior with $(\mathrm{A}-\mathrm{A}) \cap \Delta^{\perp}=0$ and a suitable nucleus $\mathrm{M} \subset \mathrm{N}$ so that the above argument applies to the kernel $l_{\mathrm{A}, \mathrm{M}}$. $\hat{\imath}$ being a projection, $\imath \mathrm{T} \subset$ Int $\imath \mathrm{A}$; so if $\psi \in E^{\prime}(\hat{\imath} \mathrm{T})$ then $\int_{\Delta} l_{\mathrm{A}, \mathrm{M}}(x-\iota y) \psi(y) d y$ defines a function in $E(\mathrm{~A})$ because $\psi \in E(\iota \mathrm{A})$. Letting $\mathrm{A}$ approach $\mathrm{T}$ and then $\mathrm{M}$ approach $N$, we obtain the desired result that $\varphi \in E^{\prime}(\hat{\imath} T)$ and $\psi=\hat{\imath} \varphi$.

What we have actually proved is an explicit interpolation formula which is to say somewhat more than is claimed in the statement of (5.9). Using Jensen's inequality one can deduce that there exists a constant $b$ depending only on $\mathrm{T}$ such that if $\varphi \in E^{\prime}(T)$ and $K(\xi)$, defined for $0 \leqq \xi \leqq \infty$ with $K(0)=0$, is nondecreasing and convex then one has the inequalities

$$
\begin{aligned}
& \int_{\Delta} K\{|\varphi(\iota y)|\} d y \leqq \int_{\Gamma} K\{b|\varphi(x)|\} d x, \\
& \int_{\Gamma} K\{|\varphi(x)|\} d x \leqq \int_{\Delta} K\{b|\varphi(\iota y)|\} d y .
\end{aligned}
$$

Here $b \leqq|\mathrm{~N}|^{-1 / 2}|\mathrm{~T}+\mathrm{N}|$ for each admissible $\mathrm{N}$. The estimate given for the

(37) See BoAs [1]. In his situation $\Gamma$ is the reals and $\Delta$ the integers. The inequalities of the paragraph are due to him. 
constant $b$ is very crude. For the best possible inequality $b$ should vary with $K$, e.g. if $K(\xi)=\xi^{2}$ one can replace $b$ by 1 in the inequalities. This is the basic result we started with, namely that the Hilbert spaces $E^{\prime}(\mathrm{T}) \cap L^{2}(\Gamma)$ and $E^{\prime}(\hat{\imath} \mathrm{T}) \cap L^{2}(\Delta)$ were isometric. However, in this instance the given interpolation formula has the defect that it appears to depend explicitly on the set $\mathrm{T}$.

At this point we want to specialize the previous situation by taking $\Delta$ to be a discrete subgroup of $\Gamma$ such that $\Gamma / \Delta$ is compact. The first task at hand is to show that $\Delta^{\perp}$ has fundamental domains of a prescribed character.

5.10. Lemma. Let $\mathrm{N}$ be a nucleus such that $\hat{\Gamma}=\Delta^{\perp}+\mathrm{N}$. Then there is a Jordan measurable subset $\mathrm{H}$ of $\mathrm{N}$ with $\hat{\mathrm{T}}=\Delta^{\perp} \oplus \mathrm{H}$.

Proof. $\Delta^{\perp} \cap(\mathrm{N}-\mathrm{N})$ is a finite set consisting of distinct points $0, t_{1}, t_{2}, \cdots$, $t_{m}$. It is easy to see that there exists an $x \in \Gamma$ such that $e\left(t_{i} x\right) \neq 1$ for each $i$. Now define a continuous invariant pseudometric $\delta$ on $\hat{\Gamma}$ by $\delta(s)=|1-e(s x)|$. For each $s \in N,\left(s+\Delta^{\perp}\right) \cap N$ is finite. Let $N_{0}$ be the collection of those points $s \in N$ such that $\delta(s) \leqq \delta\left(s^{\prime}\right)$ whenever $s^{\prime} \in N$ and $s-s^{\prime} \in \Delta^{\perp}$, and let $\mathrm{H}_{0}$ be the subset of $\mathrm{N}_{0}$ where strict inequality holds for $s \neq s^{\prime} . \widehat{\Gamma}=\Delta^{\perp}+N_{0}$ while no two points of $\mathrm{H}_{0}$ are congruent modulo $\Delta^{\perp}$. Set $d=\inf \delta\left(t_{i}\right)$. Since $\mathrm{N}_{1}=\mathrm{N}_{0} \cap \tilde{\mathrm{H}}_{0}$ is totally bounded, there are a finite number of (Jordan measurable) open balls $\Omega_{1}, \cdots, \Omega_{n}$ of diameter less than $d$ which cover $N_{1}$. Moreover no two distinct points of $\Omega_{j} \cap N$ are congruent modulo $\Delta^{\perp}$. Define inductively sets $H_{1}, \cdots, H_{n}$, $\mathrm{N}_{2}, \cdots, \mathrm{N}_{n}$ by setting $\mathrm{H}_{j}=\mathrm{N}_{j} \cap \Omega_{j}$ and putting $\mathrm{N}_{j+1}$ equal to the intersection of $\mathrm{N}_{j}$ with the complement of $\Delta^{\perp}+\mathrm{H}_{j}$. The set $\mathrm{H}=\mathrm{H}_{0} \cup \mathrm{H}_{1} \cup \ldots \cup \mathrm{H}_{n}$ has the property that $\hat{\Gamma}=\Delta^{\perp} \oplus H$. To show that the Borel set $H$ is Jordan measurable it suffices to prove that $\mathrm{H}_{0}$ and $\mathrm{N}_{1}$ are. Observe that the boundary of $\mathrm{H}_{0}$ is contained in the boundary of $\mathrm{N}_{1}$; hence we have only to show that the boundary of $N_{1}$ has Haar measure 0 . Now the boundary of $N$ has measure 0 , and $\mathrm{N}_{1}$ is relatively closed in $\mathrm{N}$; thus the problem is reduced to showing that $\left|\mathrm{N}_{1}\right|=\mid$ Int $\mathrm{N}_{1} \mid$. For each $i$, the set of points $s \in \hat{\Gamma}$ such that $\delta(s)=\delta\left(s+t_{i}\right)$ in the union of at most two disjoint cosets of $x^{\perp}$, say $s_{i}+x^{\perp}$ and $s_{i}^{\prime}+x^{\perp}$ where $e\left(2 s_{i} x\right)=e\left(t_{i} x\right)$ and $e\left(s_{i}^{\prime} x\right)=-e\left(s_{i} x\right)$. Therefore

$$
\mathrm{N}_{1}=\mathrm{N} \cap U_{i}\left[\left(s_{i}+x \perp\right) \cup\left(s_{i}^{\prime}+x \perp\right)\right] .
$$

There are two cases: either $\left|x^{\perp}\right|=0$ in which case $\left|N_{1}\right|=0$ or $\left|x^{\perp}\right|>0$ in which case $x^{\perp}$ is open and $\mathrm{N}_{1}=\operatorname{Int} \mathrm{N}_{1}$.

Henceforth take some convenient $\mathrm{H}$ arising in the above lemma; it will be of the type discussed in $\S 1$, and the considerations given there will be used here. Unfortunately we have no canonical way of picking the set $\mathrm{H}$, and it is exactly this freedom of choice which entails the arbitrariness of the succeeding constructions.

The Haar measure on $\Delta$ which leads to the conventional normalization introduced earlier is $h^{-1}$ times the counting measure. The defect in the interpolation formula of (5.9) for $L^{2}$-functions will now be remedied. 
5.11. TheOREM. The Hilbert space $L^{2}(\Delta)$ is naturally isomorphic to the Hilbert space consisting of those continuous functions $\psi \in L^{2}(\Gamma)$ for which $\hat{\psi}$ vanishes almost everywhere outside $\mathrm{H}$. For such $a \psi, h^{-1} \sum_{y \in \Delta}|\psi(x+y)|^{2}$ $=\int_{\Gamma}|\psi(z)|^{2} d z$, for each $x \in \Gamma$. The correspondence between the two Hilbert spaces is given by

$$
\psi(x)=h^{-1} \sum_{y \in \Delta} \psi(y) \chi_{\mathrm{H}}(x-y)
$$

where the series converges absolutely and uniformly on compact sets.

The only statement which is not clear from what has gone before is the one involving uniformity. Given a compact subset $\mathrm{K} \subset \Gamma$ one can choose an increasing sequence $\left\{\Delta_{n}\right\}$ of finite subsets of $\Delta$ such that for $x \in \mathrm{K}, \chi_{\mathrm{H}}(x-y)$ $=0$ unless $y \in U \Delta_{n}$. Then given $\epsilon>0$ we can find an $n(\epsilon)$ such that $h^{-1} \sum_{\nu \in \tilde{\Delta}_{n}}\left|\chi_{\mathrm{H}}(x-y)\right|^{2}<\epsilon$ for $n>n(\epsilon)$ for all $x \in \mathrm{K}$. Accordingly, $h^{-1} \sum_{y \in \Delta_{n}} \psi(y) \chi_{\mathbf{H}}(x-y)$ converges, uniformly for $x \in \mathrm{K}$, to $\psi(x)$ as $n \rightarrow \infty$. The detail that is involved here is that $\left.h^{-1} \sum_{y \in \Delta}|| \chi_{\mathrm{H}}(x-y)\right|^{2}-\left|\chi_{\mathrm{H}}\left(x^{\prime}-y\right)\right|^{2} \mid$ $=h \sum_{y \in \Delta}\left|k_{\mathrm{H}}(x-y)-k_{\mathrm{H}}\left(x^{\prime}-y\right)\right| \leqq 2 h \omega_{\mathrm{H}}\left(x-x^{\prime}\right)$ where, as before, $\omega_{\mathrm{H}}(x)$ $=\sup _{\varepsilon \in \mathrm{B}}|1-e(s x)|$. To obtain this estimate put $f(y)=\chi_{\mathrm{H}}(x-y)+\chi_{\mathrm{H}}\left(x^{\prime}-y\right)$, $g(y)=\chi_{\mathrm{H}}(x-y)-\chi_{\mathrm{H}}\left(x^{\prime}-y\right)$. The sum involved is dominated by

$$
\begin{aligned}
h^{-1} \sum_{y \in \Delta}|f(y)||g(y)| & \leqq\left\{h^{-1} \sum|f(y)|^{2}\right\}^{1 / 2} \cdot\left\{h^{-1} \sum|g(y)|^{2}\right\}^{1 / 2} \\
& =\|f\|_{2} \cdot\|g\|_{2} \leqq 2 h^{1 / 2} \cdot h^{1 / 2} \omega_{\mathrm{H}}\left(x-x^{\prime}\right) .
\end{aligned}
$$

The preceding results have related functions in $C(\Gamma)$ having certain special properties with functions in $C(\Delta)$, namely their restrictions. One is prompted to investigate the maps arising from the projection $\pi: \Gamma \rightarrow \Gamma / \Delta$. First we shall note a fairly simple result that holds for arbitrary subgroups $\Delta$; later a more powerful theorem which is valid in the special situation will be given. By way of preliminary, observe that if $\varphi \in C_{u}(\Gamma)$ and $\mathscr{T}$ is an invariant mean on $\Delta$ then $\mathfrak{M}\left(\hat{\imath} \varphi^{x}\right)$ depends only on the coset of $x$ modulo $\Delta$, i.e. it is a function $\operatorname{Tl}(\varphi)$ defined on $\Gamma / \Delta$.

5.12. Theorem. Let $\pi: \Gamma \rightarrow \Gamma / \Delta$ be a projection. Then $\hat{\pi}: C(\Gamma / \Delta) \rightarrow E^{\prime}\left(\Delta^{\perp}\right)$ is an identification map. Given any invariant mean $\mathfrak{N}$ on $\Delta$, then $\mathfrak{T}: C_{u}(\Gamma) \rightarrow$ $C_{u}(\Gamma / \Delta)$ such that $\mathfrak{\Re} \hat{\pi}$ is the identity transformation on $C_{u}(\Gamma / \Delta)$, and if $\varphi \in C_{u}(\Gamma)$, $\Lambda\{\mathfrak{T}(\varphi)\} \subset \hat{\pi}^{-1} \Lambda(\varphi)$.

Proof. The first statement is given in the first part of Reiter [2]. Given $\varphi \in C_{u}(\Gamma)$ and $\mathscr{T}$ we define $\psi(x)=\mathscr{M}\left(\hat{\imath} \varphi^{x}\right)$ where $\iota: \Delta \rightarrow \Gamma$ is the injection. $\psi \in C_{u}(\Gamma)$ and is periodic with respect to $\Delta$; if $\varphi$ were periodic we should have $\psi=\varphi$. Now if $\mu \in B(\Gamma)$ is a measure concentrated at a finite number of points one will have $(\psi, \mu)=\mathfrak{T}\{\hat{\imath}(\mu \circ \varphi)\}$. However, since $\varphi$ is assumed to be uni- 
formly continuous, $\mu \circ \varphi$, for any $\mu \in B(\Gamma)$, is a uniform limit of convolutions of the special form; hence the above equation holds for all $\mu \in B(\Gamma)$ which implies that $\Lambda(\psi) \subset \Lambda(\varphi)$. We know already that $\Lambda(\psi) \subset \Delta^{\perp}$; therefore $\Lambda(\psi)$ $\subset \Lambda(\varphi) \cap \Delta^{\perp}$ which is the same statement as $\Lambda\{\mathfrak{M}(\varphi)\} \subset \hat{\pi}^{-1} \Lambda(\varphi)$.

Unfortunately, in the above $\mathfrak{M}(\varphi)$ may be zero in a variety of circumstances for what one would consider to be trivial reasons, e.g. if $\Lambda(\varphi) \cap \Delta^{\perp}=\varnothing$. A result of a similar nature which is free from this defect is the next, where we have to assume that $\Delta$ is discrete and $\Gamma / \Delta$ compact.

5.13. TheOREM. Given $\varphi \in C(\Gamma)$, define $\varphi_{\mathrm{H}}$ by the absolutely convergent series

$$
\varphi_{\mathrm{H}}(x)=\sum_{\boldsymbol{y} \in \Delta} k_{\mathrm{H}}(x+y) \varphi(x+y) .
$$

$\varphi_{\mathrm{H}}$ has the properties:

(i) the map $C(\Gamma) \rightarrow C(\Gamma / \Delta)$ induced by $\varphi \rightarrow \varphi_{\mathrm{H}}$ is a left inverse of $\hat{\pi}: C(\Gamma / \Delta)$ $\rightarrow C(\Gamma)$ induced by the projection $\pi: \Gamma \rightarrow \Gamma / \Delta$,

(ii) $\left\|\varphi_{\mathrm{H}}\right\| \leqq\|\varphi\|$ and $\varphi \geqq 0$ implies $\varphi_{\mathrm{H}} \geqq 0$,

(iii) $\left|\varphi(x)-\varphi_{\mathrm{H}}(x)\right| \leqq 2\|\varphi\| \cdot\left[1-k_{\mathrm{H}}(x)\right] \leqq 2\|\varphi\| \omega_{\mathrm{H}}(x)$,

(iv) $\Lambda\left(\varphi_{\mathrm{H}}\right) \subset\left[\Lambda(\varphi)+\mathrm{H}^{0}\right] \cap \Delta^{\perp}$,

(v) if $\varphi$ has compact spectrum, $\varphi_{\mathrm{H}}=h \sum_{t \in \Delta} \perp \Phi_{\mathrm{H}}(t) e_{t}$,

(vi) if $\varphi$ is positive definite so is $\varphi_{\mathrm{H}}$.

Proof. Utilizing (5.10) and (5.11), one sees that $\sum k_{\mathrm{H}}(x+y)$ $=h^{-2} \sum\left|\chi_{\mathrm{H}}(x+y)\right|^{2} \equiv 1$ and that the sum converges uniformly on compact sets. Thus $\varphi_{\mathrm{H}}$ is continuous; it is obviously periodic. (i), (ii), and (iii) are trivial once one has the properties of $k_{\mathrm{H}}$ established in $\$ 1$. (v) follows by applying the Poisson summation formula to $\Phi_{\mathrm{H}}(t)=\int e(t x) k_{\mathrm{H}}(x) \varphi(x) d x$ and yields (iv) as a by-product. The last assertion is equally simple. For any $\alpha \in B(\hat{\Gamma})$, define $\alpha_{\mathrm{H}}$ on compact subsets $\mathrm{K} \subset \hat{\Gamma}$ by $\alpha_{\mathrm{H}}(\mathrm{K})=h \sum \int \hat{k}_{\mathrm{H}}(t-s) \alpha(d s)$, the sum extending over all lattice points $t \in \Delta^{\perp} \cap \mathrm{K} . \alpha \rightarrow \alpha_{\mathrm{H}}$ is a norm-diminishing, positivity preserving projection of $B(\hat{\Gamma})$ onto $B\left(\Delta^{\perp}\right)$, and if $\varphi$ is the Fourier-Stieltjes transform of $\alpha$ then $\varphi_{\mathrm{H}}$ is the transform of $\alpha_{\mathrm{H}}$.

The main interest in (5.13) arises when the group $\Gamma$ possesses a family $\{\Delta\}$ of discrete subgroups with compact factor groups such that the corresponding family $\{\mathrm{H}\}$ is a $J$-net. In this case $\varphi_{\mathrm{H}}$ converges to $\varphi$ as $\mathrm{H} \rightarrow 0$; this is the explicit construction mentioned in the remarks following (5.5). If $\Omega$ is an open subset of $\hat{\Gamma}$ and $\Lambda(\varphi)$ is a compact subset of $\Omega$ then for sufficiently small $\mathrm{H}, \Lambda\left(\varphi_{\mathrm{H}}\right) \subset \Omega_{0}=\mathrm{U}\left(\Omega \cap \Delta^{\perp}\right)$ where the union is taken over the family $\{\Delta\}$.

We now wish to interpret some of the previous theorems in the light of the theory of entire functions of exponential type. The setting for the connection between spectral theory and the theory of functions of a complex variable may be carried over quite easily to general groups by means of the 
study of one-parameter subgroups $\left({ }^{38}\right)$. Here a one-parameter subgroup of $\Gamma$ means simply a nontrivial representation $\rho: R \rightarrow \Gamma$. Corresponding to it is a representation $\hat{\rho}: \hat{\Gamma} \rightarrow \hat{R}$; we shall normalize the duality relationship by taking the Haar measure in $R$ as $(2 \pi)^{-1}$ times Lebesgue measure and the Haar measure in $\hat{R}$ as the Lebesgue measure; this determines the characters up to complex conjugation; given $\xi \in R, t \in \hat{R}$ we set $e(t \xi)=\exp (i t \xi)$.

5.14. Theorem. Let $\rho: R \rightarrow \Gamma$ be a one-parameter subgroup and suppose $\varphi \in C(\Gamma)$. Set $-\tau=\inf \hat{\rho} t$ where the infimum is taken over $t \in \Lambda(\varphi)$. Then

(i) $\tau<\infty$ if and only if each of the functions $\hat{\rho} \varphi^{x}$ is the boundary value of $a$ function analytic of exponential type $\leqq \tau$ in the upper half-plane,

(ii) writing, in case $\tau<\infty, \varphi(x+\rho \zeta)$ for the value at $\zeta, \operatorname{Im} \zeta \geqq 0$, of the analytic function with boundary value $\hat{\rho} \varphi^{x}$, we have $|\varphi(x+\rho \zeta)| \leqq\|\varphi\| \exp \{\tau \operatorname{Im} \zeta\}$,

(iii) $\tau=\sup \lim \sup _{\eta \rightarrow \infty} \eta^{-1} \log |\varphi(x+\rho i \eta)|$ where the supremum is taken over $x \in \Gamma_{\rho}^{\prime}, \Gamma_{\rho}^{\prime}$ being any set such that $\rho R+\Gamma_{\rho}^{\prime}$ is dense in $\Gamma$.

Proof. Once the theorem is known for the identity representation of $R$ on $R$ the general case is clear consequence of (5.6). Although the classical situation is well-known, I should like to give a proof emphasizing the viewpoint of spectral theory. Given $\varphi$ bounded on the real axis we form the Poisson integral for $\operatorname{Im} \zeta>0$,

$$
\varphi(\zeta)=\frac{1}{2 \pi i} \int \varphi(x)\left\{\frac{1}{x-\zeta}-\frac{1}{x-\zeta}\right\} d x
$$

where $d x$ is the Lebesgue measure. $\varphi(\zeta)$ is analytic if and only if $\partial \varphi / \partial \bar{\zeta}=0$, and $\partial \varphi / \partial \bar{\xi}$ at $\xi+i \eta$ is $f_{\eta} \circ \varphi(\xi)$ where $f_{\eta}(x)=i(x-i \eta)^{-2} \cdot f_{\eta}(t)=0$ for $t \geqq 0$ and is nonvanishing in $t<0$. Hence $f \eta \circ \varphi=0$ implies $\Lambda(\varphi) \subset[0, \infty]$, and the converse is also true. Thus, given $\varphi \in C(R)$, the Poisson integral $\varphi(\zeta)$ defined for $\operatorname{Im} \zeta>0$ is analytic there if and only if $\tau \leqq 0$. Since, in any case, $\varphi(x+i \eta)$ $\rightarrow \varphi(x)$ uniformly on compact sets as $\eta \rightarrow 0$ and $|\varphi(\xi+i \eta)| \leqq\|\varphi\|$, if $\tau \leqq 0$ then $\varphi$ is the boundary value of a bounded analytic function in the upper halfplane. In the other direction, suppose $\varphi$ is the boundary value of a function $\psi$ which is bounded and analytic in the upper half-plane, i.e. for some sequence of $\eta$ 's tending to 0 from above, $\psi(x+i \eta)$ converges to $\varphi(x)$ for almost all $x$. It is easy to see that $\psi$ is represented by its Poisson integral:

$$
\psi(\zeta+i \eta)=\frac{1}{2 \pi i} \int \psi(x+i \eta)\left\{\frac{1}{x-\zeta}-\frac{1}{x-\zeta}\right\} d x \quad \text { for } \eta, \operatorname{Im} \zeta>0 .
$$

(38) For a treatment of analytic functions on groups see ARENS and SiNGER and the references given there. Arens and Singer develop their theory with reference to a semi-group in $\hat{\Gamma}$ which is not given in a natural way; so that analytic functions are not defined intrinsically on general groups. Plancherel and Polya deal with entire functions of several variables by the methods of harmonic analysis; their work was the original model for much of the contents of this section. 
Letting $\eta$ approach 0 and using dominated convergence we conclude $\psi(\zeta)$ $=\varphi(\zeta)$. Therefore $\varphi(\zeta)$ is analytic which, as we noted above, implies $\tau \leqq 0$. The proof of (i) and (ii) for $\tau=0$ is complete, and the result follows immediately for arbitrary $\tau$. (iii) is equivalent to (ii), with the best possible choice of $\tau$, by the Phragmen-Lindelöf theorem.

It should be noted that if $\{\pi(\eta)\}$ is the ordinary Cauchy-Poisson semigroup on $R$, i.e. $\pi(\eta ; d \xi)=\pi^{-1} \eta\left(\xi^{2}+\eta^{2}\right)^{-1} d \xi$, then $\{\rho \pi(\eta)\}$ is the semi-group on $\Gamma$ giving the Poisson integral; that is, if $\hat{\rho}(t) \geqq 0$ for $t \in \Lambda(\varphi)$ where $\varphi \in C(\Gamma)$ then $\varphi(x+\rho i \eta)=\rho \pi(\eta) \circ \varphi(x)$. The negative definite function on $\hat{\Gamma}$ corresponding to this semi-group is $\nu=|\hat{\rho}|$.

A good theory of analytic functions is available only for connected groups: if $\Gamma$ is not connected it has a proper open subgroup and hence nontrivial characters which are identically 1 on open sets. The characters ought to be "entire." One of the essential properties of entire functions, or for that matter analytic ones, is that they are completely determined by their values on any open set. Conversely, in a connected group one has a dense collection of oneparameter subgroups; this enables one to develop a theory of analytic functions. Now our aim is not to consider analytic functions on connected groups per se but only certain topics in the subject which are illuminated by the viewpoint of spectral theory. For this reason we shall not strive for maximum generality.

If $\Gamma$ is a connected group, we shall call $\varphi \in C(\Gamma)$ "entire" if $\Lambda(\varphi)$ is compact. For each one-parameter subgroup $\rho, \varphi(x+\rho \zeta)$ is then an entire function of exponential type in the complex variable $\zeta . \varphi$ cannot vanish on an open set without being identically 0 since the one-parameter subgroups are dense in $\Gamma$. The notion of "type" can be extended with a slight modification; the type of a function of one complex variable will be an interval instead of a number. Generally we define the type of $\varphi, T(\varphi)$, to be the convex hull of $\Lambda(\varphi)$, i.e. the set of points $t \in \hat{\Gamma}$ such that $\inf _{s \in \Delta(\varphi)} \hat{\rho} s \leqq \hat{\rho} t \leqq \sup _{u \in \Lambda(\varphi)} \hat{\rho} u$ for each one-parameter subgroup $\rho$. Reasoning analogous to that used in (5.14) may be applied to entire functions. In addition one has a simple theory of differential operators $\left({ }^{39}\right)$. Given $\rho$ a one-parameter subgroup and $\varphi$ an entire function we define $\partial \rho \circ \varphi(x)=\partial \varphi(x+\rho \xi) / i \partial \xi$ at $\xi=0$. The Fourier transform of $\partial \rho$ is $\hat{\rho}$. From a classical result of $\mathrm{S}$. Bernstein one obtains the theorem that $V_{\mathrm{T}}(\hat{\rho})=\sup _{t \in \mathrm{T}}|\hat{\rho}(t)| ;$ this is the same statement as $\|\partial \rho \circ \varphi\| \leqq \tau\|\varphi\|$ where $\tau=\sup |\hat{\rho}(t)|$, the supremum taken over $t \in \Lambda(\varphi)$. As a consequence one has that if $F\left(\zeta_{1}, \cdots, \zeta_{n}\right)$ is an entire function of $n$ complex variables and $\rho_{1}, \cdots, \rho_{n}$ are one-parameter subgroups then if we form

$$
\lambda(t)=F\left[\hat{\rho}_{1}(t), \cdots, \hat{\rho}_{n}(t)\right], \lambda \in V(\mathrm{~T})
$$

for each compact T. $\lambda$ corresponds to an operator $L$ which acts on all entire functions; the operator $L$ may be taken literally as $L=F\left(\partial \rho_{1}, \cdots, \partial \rho_{n}\right)$

(39) Cf. RIss. 
where the latter expression is understood as a power series. There is an operational calculus for a much wider class of $F^{\prime}$ s, e.g. those corresponding to fractional differential operators which include many generalized Laplacians. However it is also interesting to consider more restricted $F$ 's at the gain of enlarging the domain of $L$. We shall confine our attention to the case where $F$ is a polynomial, and hence $L$ is a "differential polynomial." We shall sa $y$ that $\psi \in C(\Gamma)$ is in the domain of $L$ if all the appropriate partial derivatives are continuous and the net result, $L \circ \psi$, is bounded.

5.15. Theorem $\left({ }^{40}\right)$. Let $L$ be a differential polynomial on the connected group $\Gamma$. Set $\lambda(t)=L \circ e_{t}(0)$ and $\Lambda_{a}=\{t|| \lambda(t) \mid \leqq a\}$. Suppose $\varphi \in C(\Gamma)$ and each of the functions $\varphi_{m}$ is in the domain of $L$ where $\varphi_{0}=\varphi$ and $\varphi_{m+1}=L \circ \varphi_{m}$. Then $\lim \sup _{m \rightarrow \infty}\left\|\varphi_{m}\right\| \|^{1 / m} \leqq a$ implies $\Lambda(\varphi) \subset \Lambda_{a}$.

Proof. It is sufficient to consider only $\Gamma=R^{n}$ since the general case then follows easily via (5.6). Let $f$ be a continuous function, vanishing outside a compact set, which is in the domain of $L$. It is then a trivial verification that if $\psi$ is in the domain of $L$ one has also $f \circ \psi$ in the domain of $L$ and $L \circ(f \circ \psi)$ $=f \circ(L \circ \psi)=(L \circ f) \circ \psi$. Now let $g=L \circ f$ and denote by $f_{m}, g_{m}$ the respective $m$-fold convolutions. We assert that $g_{m} \circ \varphi=f_{m} \circ \varphi_{m}$; this is proved by induction. It is an identity for $m=0$; suppose it is true for a given $m$. $f_{m+1} \circ \varphi_{m+1}=f_{m} \circ\left\{f \circ\left(L \circ \varphi_{m}\right)\right\}=f_{m} \circ\left\{(L \circ f) \circ \varphi_{m}\right\}=(L \circ f) \circ\left(f_{m} \circ \varphi_{m}\right)$ $=g \circ\left(g_{m} \circ \varphi\right)=g_{m+1} \circ \varphi$. Now we observe that (4.3) is true without the assumption that $\Lambda(\varphi)$ is compact provided we deal with absolutely continuous measures. Also $\hat{g}(t)=\lambda(t) \hat{f}(t)$. Hence $\sup _{t \in \Delta(\varphi)}|\lambda(t)||\hat{f}(t)|=\sup _{t \in \Lambda(\varphi)}|\hat{g}(t)|$ $=\lim \sup _{m \rightarrow \infty}\left\|g_{m} \circ \varphi\right\|^{1 / m}=\lim \sup _{m \rightarrow \infty}\left\|f_{m} \circ \varphi_{m}\right\|\left\|^{1 / m} \leqq\right\| f\left\|_{1} \cdot \lim \sup _{m \rightarrow \infty}\right\| \varphi_{m}\|\|^{1 / m}$. For a given $t$ we can choose $f$ suitably so that $\|f\|_{1}=1=\hat{f}(t)$; this gives $\sup _{t \in \Delta(\varphi)}|\lambda(t)| \leqq \lim \sup _{m \rightarrow \infty}\left\|\varphi_{m}\right\| \|^{1 / m}$.

If $L$ is such that $\Lambda_{a}$ is compact for each finite $a$ we say that " $L$ is elliptic." In this case we know from (4.3) that $\Lambda(\varphi) \subset \Lambda_{a}$ if and only if $\lim \sup _{m \rightarrow \infty}\left\|\varphi_{m}\right\|^{1 / m}$ $\leqq a$. This result may be viewed as a generalization of the theorem of $\mathrm{S}$. Bernstein where $\Gamma=R^{1}$ and $L$ is ordinary differentiation which gives a necessary and sufficient condition for $\varphi$ to be entire of exponential type $\leqq a$. In statements of this type the connectedness of the group $\Gamma$ enters only on account of our insistence that $L$ be a differential operator. We shall now indicate some theorems which are classically stated in terms of entire functions $\left({ }^{41}\right)$ but which have generalizations to functions with compact spectrum on groups which need not be connected. An example of what we have in mind is Carlson's Theorem. Here we take $\Gamma=R^{1}$ and $\Delta$ the subgroup of integers. The statement that $\varphi$ is of exponential type $<\pi$ implies, assuming $\varphi$ is bounded, that $\varphi \in E^{\prime}(\mathrm{T})$ where the hypotheses of (5.9) are fulfilled. The superfluous boundedness assumption may be removed by Cartwright's Theorem. Having

$\left({ }^{40}\right)$ This theorem and its proof are more or less contained in $\$ 3$ of Domar. However, I think it is worth emphasizing.

(11) BoAs [2] is a convenient reference. 
this, the statement that the map $\hat{\imath}: E^{\prime}(\mathrm{T}) \rightarrow E^{\prime}(\hat{\imath} \mathrm{T})$ is one-to-one is precisely Carlson's Theorem. The explicit interpolation formula has been given by Boas. (5.9) together with the inequalities given after it may be interpreted in the case $\Gamma=R^{r}$ to give results very close to those of Plancherel and Polya. Again, in the case $\Gamma=R^{1}, \Delta=I^{1}$ we may take $H=(-\pi, \pi]$ in (5.10). (5.11) is then the classical Valiron interpolation formula. When $\Gamma=R^{1}, \Delta=$ integral multiples of $2 \pi h^{-1}$ and $\mathrm{H}=(-h / 2, h / 2]$ the function $\varphi_{\mathrm{H}}$ given in (5.13) is $\varphi_{\mathrm{H}}(x)=\varphi(0)$, when $x \in \Delta$ and

$$
\varphi_{\mathrm{H}}(x)=4 h^{-2} \sin ^{2} h x / 2 \sum_{\nu=-\infty}^{\infty}\left(x+2 \pi h^{-1} \nu\right)^{-2} \varphi\left(x+2 \pi h^{-1} \nu\right),
$$

otherwise. If $\varphi$ is entire of exponential type $\tau$ and is bounded on the real axis, $\varphi_{\mathrm{H}}$ is the Levitan polynomial $\left({ }^{42}\right): \varphi_{\mathrm{H}}(x)=h \sum_{\nu=-n}^{n} \Phi_{\mathrm{H}}(\nu h) \exp (i \nu h x)$ where $n$ is the smallest integer not less than $h^{-1} \tau$. We have $\left|\varphi(x)-\varphi_{\mathrm{H}}(x)\right| \leqq 2\|\varphi\|$ $\cdot\left[1-(h x / 2)^{2} \sin ^{2} h x / 2\right] \leqq 2\|\varphi\| \min \left(1, h^{2} x^{2} / 12\right)$. The Levitan polynomials can be generalized to entire functions on any connected group because if $\Gamma$ is connected there always exists a family $\{\Delta\}$ of discrete subgroups with compact factor group such that the corresponding family $\{\mathrm{H}\}$ is a $J$-net.

Finally we shall give the proof of Theorem (4.5). The details here may serve as good examples of applications of the techniques developed in this section.

Proof of 4.5. First we show that if $\Gamma$ has rank $r \geqq 3$ then spectral synthesis does not hold locally. We start out with the case $\Gamma_{0}=I^{3}$ where there exists a compact set $\mathrm{T}_{0}$ in $\hat{\Gamma}_{0}$ and a $\mu_{0} \in B(\Gamma)$ with compact support such that $\hat{\mu}_{0}=0$ on $\mathrm{T}_{0}$ but $V_{\mathrm{T}_{0}}^{\prime}\left(\hat{\mu}_{0}\right) \neq 0$. Now let $\Gamma_{1}$ be a discrete group of rank $\geqq 3$; there is then an injection $\rho: \Gamma_{0} \rightarrow \Gamma_{1}$. Let $\mu_{1}=\rho \mu_{0}, T_{1}=\hat{\rho}^{-1} T_{0} . \mu_{1}$ has compact support, $T_{1}$ is compact, and $V_{T_{1}}^{\prime}\left(\hat{\mu}_{1}\right) \geqq V_{T_{0}}^{\prime}\left(\hat{\mu}_{0}\right)>0$ by (5.3). Next suppose $\Gamma_{2}$ is compactly generated; $\Gamma_{2}$ has a discrete subgroup $\Gamma_{1}$ such that $\Gamma_{2} / \Gamma_{1}$ is compact; let $\iota: \Gamma_{1} \rightarrow \Gamma_{2}$ be the natural injection. $\Gamma_{1}$ has the same rank as $\Gamma_{2}$; we assume rank $\Gamma_{2} \geqq 3$. Let $\mu_{2}=\imath \mu_{1}$ and let $T_{2}$ be a compact subset of $\hat{\Gamma}_{2}$ such that $\imath \mathrm{T}_{2}=\mathrm{T}_{1}$ and $\hat{\imath}$ is one-to-one on a neighborhood of $\mathrm{T}_{2} . V_{\mathbf{T}_{1}}^{\prime}\left(\hat{\mu}_{1}\right) \neq 0$ implies $V_{\mathbf{T}_{2}}^{\prime}\left(\hat{\mu}_{2}\right) \neq 0$. Lastly, if $\Gamma$ is any group of rank $\geqq 3$ there is a compactly generated open subgroup $\Gamma_{2}$ which has rank $\geqq 3$. Suppose $\sigma: \Gamma_{2} \rightarrow \Gamma$ is the natural injection. Set $\mu=\sigma \mu_{2}, \mathrm{~T}=\hat{\sigma}^{-1} \mathrm{~T}_{2} . \mu$ has compact support, $\mathrm{T}$ is compact, $\hat{\mu}=0$ on $\mathrm{T}$, but $V_{\mathbf{T}}^{\prime}(\hat{\mu})>0$.

To prove the affirmative part of (4.5) we shall need a construction. Let $\Gamma$ be a compactly generated group of rank $r$. $\Gamma$ has a discrete subgroup $\Delta \simeq I^{r}$ and a symmetric compact subset $\mathrm{K}$ containing the generators of $\Delta$ such that $\Gamma=\Delta+\mathrm{K}$. Each of the sets $\left\{t \in \hat{\Gamma}\left|\sup _{x \in \mathrm{K}}\right| 1-e(t x) \mid<4 n^{-1}\right\}$ contains a nucleus $\mathrm{N}_{n}$ with $(n \Delta)^{\perp}+\mathrm{N}_{n}=\hat{\mathrm{\Gamma}}$. Let $\mathrm{H}_{n}$ be the set constructed in (5.10) so that $\mathrm{H}_{n} \subset \mathrm{N}_{n}$ and $(n \Delta)^{\perp} \oplus \mathrm{H}_{n}=\hat{\mathrm{\Gamma}}$. It is easy to see that $h_{n}=n^{-r} h_{1}$. Let $\omega_{n}(x)$

(42) Cf. Achieser, p. 146. (5.13) was proved for the case under consideration by M. Krein; however the proof given here, when stripped down for the real line seems a little bit simpler and sharper. 
$=\sup |1-e(t x)|$ where the supremum is taken over $t \in \mathrm{H}_{n}$. Write $\mathrm{K}_{1}=\mathrm{K}, \mathrm{K}_{j+1}$ $=\mathrm{K}_{j}+\mathrm{K}$. Since $\Gamma=\mathrm{UK}_{j}$ we can define for each $x$ a gauge $\mathrm{K}(x)$, the smallest value of $j$ such that $x \in \mathbf{K}_{j}$. Clearly $\omega_{n}(x)<4 n^{-1} \mathrm{~K}(x)$. Put $\delta(x)=\sup _{n} n^{r / 2} \omega_{n}^{r / 2}(x)$. $\delta$ is a pseudometric with $\delta(n x)=O\left(n^{r / 2}\right)$ for fixed $x$. For $r \leqq 2, n^{r / 2} \omega_{n}(x)$ $\leqq 1+\delta(x)$. It follows that in this case if $\mu \in B(\Gamma)$ and $\int[1+\delta(x)]|\mu(d x)| \leqq M$ then $|\hat{\mu}(s)-\hat{\mu}(t)| \leqq 2 M h_{1}^{-1 / 2} h_{n}^{1 / 2}$ whenever $s-t \in \mathrm{H}_{n}^{0}$.

It is now an immediate consequence of (4.4) that if $\mathrm{T}$. is compact, $\int[1+\delta(x)]|\mu(d x)|<\infty$, and $\hat{\mu}=0$ on $\mathrm{T}$ then $V_{\mathrm{T}}^{\prime}(\hat{\mu})=0$. (Let $\left\{\mathrm{H}_{n}\right\}$ be the $J$-net in question.) For $\varphi \in C(\Gamma)$ define $\left|\|\varphi\| \|=\sup _{x}\right| \varphi(x) \mid[1+\delta(x)]^{-1}$. The continuous linear functionals in this topology are precisely those of the form $F(\varphi)=\int \varphi(-x) \mu(d x)$ where $\mu$ satisfies the condition given above. Hence spectral synthesis holds in this topology. Spectral analysis does also; if $\delta(x)$ is a pseudometric with $\sum n^{-2} \log [1+\delta(n x)]<\infty$ for each $x \in \Gamma$ then given a point $t \in \hat{\Gamma}$ and a closed set $\mathrm{T} \subset \hat{\Gamma}$ with $t \in \mathrm{T}$ there is an (absolutely continuous) measure $\mu$ such that $\int[1+\delta(x)]|\mu(d x)|<\infty, \hat{\mu}(t)=1$, and $\hat{\mu}=0$ on $\mathrm{T}$.

The passage to the general case is very easy. Let $\Gamma$ be any group of rank $r \leqq 2$. For each compactly generated open subgroup we have a pseudonorm as given above. These pseudonorms define a topology weaker than the strict topology and stronger than the topology of uniform convergence on compact sets in which spectral synthesis holds; spectral analysis does likewise, for if $t$ is a point of $\hat{\Gamma}$ and $\mathrm{T}$ a closed subset not containing it, one can find a $\mu \in B(\Gamma)$ with compactly generated support, satisfying the appropriate growth condition whose Fourier-Stieltjes transform separates $t$ and T.

6. Examples of spectral synthesis. In this section we shall exhibit a number of sets which admit spectral synthesis. The examples essentially deal with multiple Fourier transforms in Euclidean space, $\Gamma=R^{r}$; however they are all capable of being stated in a more general context, and, in particular, dimensionality considerations do not play any rôle.

As far as the spectral synthesis problem is concerned it is unnecessary to consider the most general locally compact abelian groups. First of all, one can assume that $\Gamma$ is discrete. For example, the spectral synthesis problem in an arbitrary compactly generated group of rank $r$ is equivalent to the problem for $I^{r}$. The validity of these assertions may be checked by making suitable use of (5.7), (5.8), and (5.9). The consequence of (5.7) and (5.8) which was the main aim of REITER [2] is

6.1. TheOREM. Let $\rho: \Gamma_{1} \rightarrow \Gamma_{2}$ be a homomorphism and $\mathrm{T}_{1}$ and $\mathrm{T}_{2}$ respective closed subsets of $\hat{\Gamma}_{1}$ and $\hat{\Gamma}_{2}$ such that $\mathrm{T}_{1}=\hat{\rho} \mathrm{T}_{2}$ and $\mathrm{T}_{2}=\hat{\rho}^{-1} \mathrm{~T}_{1}$. Then $\mathrm{T}_{1}$ is an $S$-set if and only if $\mathrm{T}_{2}$ is an $S$-set.

The reduction of the spectral synthesis problem to discrete groups shows that in some sense the question is purely algebraic. However, this reduction may not be the most useful one. Usually it will be convenient to take $R^{r}$ for the representative of the compactly generated groups of rank $r$. One of the reasons is that in Euclidean space a homothety of a set $\mathrm{T}$ gives rise to a par- 
ticularly simply transformation of $E^{\prime}(\mathrm{T})$. Thus it is nearly a triviality that spectral synthesis holds for star-shaped domains in $R^{r}$ : assuming that $\mathrm{T}$ is star-shaped with respect to the origin and is the closure of its interior, for $\varphi \in E^{\prime}(\mathrm{T})$ and $0 \leqq \xi<1$ we have $\varphi_{\xi} \in E(\mathrm{~T})$ where $\varphi_{\xi}(x)=\varphi(\xi x)$; since $\varphi_{\xi} \rightarrow \varphi$ narrowly as $\xi \rightarrow 1, \varphi \in E(\mathrm{~T})$.

One of the sources of difficulty in the spectral synthesis question is that the class of $S$-sets is not closed under the ordinary set operations. For example, if in $R^{3}$ we take $T_{1}=\{t|| t \mid \leqq 1\}$ and $T_{2}=\{t|| t \mid \geqq 1\}$ both are $S$-sets by the argument given in the paragraph above (use $1<\xi<\infty$ for $\mathrm{T}_{2}$ ). However $\mathrm{T}_{1} \cap \mathrm{T}_{2}$ is not an $S$-set. It is not known whether the class of $S$-sets is closed under finite unions. Below we shall give three methods for exhibiting $S$-sets. The procedures are due to Calderón, Kahane and Salem, and Herz.

The basic idea of Calderón $\left.{ }^{43}\right)$ is to introduce a property of sets which is stronger than spectral synthesis but which is easily seen to be preserved by the formation of finite unions and by injections.

Definition. A subset $\mathrm{T}$ of $\hat{\Gamma}$ is of type $S S$ if the hypotheses $\varphi \in C_{u}(\Gamma)$, $\mu \in B(\Gamma), \hat{\mu}(t)=0$ for $t \in \mathrm{T}$, and $\Lambda(\mu \circ \varphi) \subset \mathrm{T}$ imply $\mu \circ \varphi=0$.

The most important facts about $S S$-sets may be summarized together.

6.2. Theorem. (i) $\mathrm{T}$ is an SS-set if the boundary of $\mathrm{T}$ is an SS-set. (ii) The countable union of closed SS-sets is an SS-set. (iii) The injective image of an SS-set is an SS-set.

Proof. Using the assumptions and notation of the above definition, $\Lambda(\mu \circ \varphi) \subset$ boundary of $\mathrm{T}$, so (i) is quite trivial. Suppose $\mathrm{T}=\mathrm{U}_{n=1}^{\infty} \mathrm{T}_{n}$ where each $\mathrm{T}_{n}$ is a closed $S S$-set. If $\Lambda(\mu \circ \varphi)$ were not empty, there would exist, by Baire's category theorem, an open set $\Omega$ and a point $t$ such that $t \in \Lambda(\mu \circ \varphi)$ $\cap \Omega \subset \mathbf{T}_{n}$ for some $n$. Choosing $f \in L^{1}(\Gamma)$ such that $\hat{f}(t) \neq 0$ while $\hat{f}$ vanishes outside $\Omega$, one sees that the function $f \circ \varphi$ and the measure $\mu$ would together furnish a contradiction to the assumption that $\mathrm{T}_{n}$ is of type $S S$, -(ii) is proved. The situation in (iii) is that we are given a subgroup $\Delta$ of $\Gamma$ with the projection map $\pi: \Gamma \rightarrow \Gamma / \Delta, \mathrm{T}$ is supposed to be an $S S$-set in the character group of $\Gamma / \Delta$, and $\hat{\pi} T$ is under investigation. This is precisely the situation to which (5.12) applies. Let $\mathfrak{T}$ be some invariant mean on $\Delta$, and let us apply the hypotheses of the definition of an $S S$-set to $\hat{\pi} \mathrm{T} . \Lambda(\mu \circ \varphi) \subset \hat{\pi} \mathrm{T}$, so $\mu \circ \varphi$ $\in \hat{\pi} E^{\prime}(\mathrm{T})$ and $\mu \circ \varphi=\hat{\pi} \mathfrak{T}(\mu \circ \varphi)$. The same basic uniformity argument used in the proof of (5.12) shows that $\mathfrak{T}(\mu \circ \varphi)=\pi \mu \circ \mathfrak{M}(\varphi)$. However $\mathfrak{M}(\varphi)$ $\in C_{u}(\Gamma / \Delta), \pi \mu \in B(\Gamma / \Delta), \pi \hat{\mu}$ vanishes on $\mathrm{T}$ (since $\hat{\mu}=0$ on $\hat{\pi} \mathrm{T}$ ), and $\Lambda\{\pi \mu \circ \mathfrak{N}(\varphi)\} \subset \mathrm{T}$. T being of type $S S$, these imply $\mathbb{M}(\mu \circ \varphi)=0$, on the strength of which we conclude that $\mu \circ \varphi=0$, and this proves (iii). The chief consequence of the theorem is

6.3. Corollary. Polyhedral sets in $R^{r}$ are SS-sets and a fortiori sets for which spectral synthesis holds.

(4) See Ideals in abelian group algebras in SyMposium. 
This corollary is more a matter of definition than anything else. Polyhedral sets are defined inductively: each subset of $R^{0}$ is polyhedral; a set in $R^{r}, r \geqq 1$, is polyhedral if it is closed and its boundary is the finite (one could be more general and allow countable) union of translates of the images of polyhedral sets in $R^{r-1}$. Unfortunately, this procedure gives the only known method for constructing closed $S S$-sets, while on the other hand, an example of an $S$-set which is not an $S S$-set is lacking. One would like to have statements (6.2) (i) and (ii) with " $S S$ " replaced by " $S$ ". In lieu of these there is a principle implicit in the work of Calderón which allows one to piece together $S$-sets.

6.4. Theorem. Given $\mathrm{T}$, suppose there is an SS-set $\Sigma \subset \mathrm{T}$ such that each point of $\mathrm{T} \cap \tilde{\Sigma}$ is known to be relatively interior in $\mathrm{T}$ to a subset of $\mathrm{T}$ which is an $S$-set. Then $\mathrm{T}$ is an $S$-set.

Proof. Suppose $\varphi \in C(\Gamma), \Lambda(\varphi) \subset \mathrm{T}, \mu \in B(\Gamma)$, and $\hat{\mu}=0$ on T. If $t \in \mathrm{T} \cap \Omega$ $\subset \mathrm{T}^{\prime} \subset \mathrm{T}$ where $\Omega$ is open and $\mathrm{T}^{\prime}$ is an $S$-set, then we may choose an $f \in L^{1}(\Gamma)$ with $\hat{f}(t) \neq 0$ and $\hat{f}=0$ outside $\Omega . \Lambda(f \circ \varphi) \subset \mathrm{T}^{\prime}$ while $\hat{\mu}=0$ on $\mathrm{T}^{\prime}$, an $S$-set, so that $\mu \circ f \circ \varphi=0$, i.e. $t \notin \Lambda(\mu \circ \varphi)$. Thus the hypotheses of the theorem guarantee that $\Lambda(\mu \circ \varphi) \subset \Sigma$. Since $\Sigma$ is an $S S$-set, $\mu \circ \varphi=0$.

An example of the foregoing is given at the end of this section. An entirely different set of examples is provided by the device of KAHANE and SAlEm. Their purpose was to exhibit perfect sets $\mathrm{T}$ such that $E^{\prime}(\mathrm{T})$ consisted entirely of Fourier-Stieltjes transforms. However, a minor modification of their idea leads to the ad hoc construction of $S$-sets in any $\sigma$-compact group of finite rank.

Construction. Let $\Gamma$ be a $\sigma$-compact group of rank $r<\infty$. Suppose a finite subset, $\mathrm{T}_{1}$, of $\hat{\Gamma}$ is given together with a constant $m>1$. A sequence $\mathrm{T}_{2}, \mathrm{~T}_{3}, \cdots$ of finite sets can be constructed so that if $\mathrm{T}$ is the closure of $\bigcup_{n=1}^{\infty} \mathrm{T}_{n}$ and $\varphi \in E^{\prime}(\mathrm{T})$ then there is a sequence $\left\{\varphi_{n}\right\}$ with $\varphi_{n} \in E\left(\mathrm{~T}_{n}\right)$ such that $\left\|\varphi_{n}\right\| \leqq m\|\varphi\|$ and $\varphi_{n} \rightarrow \varphi$, uniformly on compact sets, as $n \rightarrow \infty$. The only limitation on the sets $\mathrm{T}_{n}$ is the requirement that $\mathrm{T}_{n} \subset \mathrm{T}_{n+1} \subset \mathrm{T}_{n}+\mathrm{H}_{n}^{0}$ where $\mathrm{H}_{n}$ is determined by $\mathrm{T}_{n}$ and $\left\{\mathrm{H}_{n}\right\}$ forms a $J$-net. We suppose, by induction, that $\mathrm{T}_{n}$ has been defined and that $\mathrm{H}$ is some $J$-set with $\left|\mathrm{H}^{0}+\mathrm{H}^{0}\right| \leqq 4^{r} h$. If $\varphi \in C(\Gamma)$ and $\Lambda(\varphi) \subset \mathrm{T}_{n}+\mathrm{H}^{0}$ then $k_{\mathrm{H}}(x) \varphi(x)=\int e(t x) \Phi_{\mathrm{H}}(t) d t$ where $\Phi_{\mathrm{H}}$ vanishes outside the set $\mathrm{T}_{n}+\mathrm{H}^{0}+\mathrm{H}^{0}$. Let $\varphi_{n}(x)$ be the function obtained by replacing $e(t x)$ in the integral with $e[s(t) x]$ where $s(t)$ is a measurable function having its values in $\mathrm{T}_{n}$ so that $t-s(t) \in \mathrm{H}^{0}+\mathrm{H}^{0} . \varphi_{n} \in E\left(\mathrm{~T}_{n}\right)$ and

$$
\begin{aligned}
\left|k_{\mathrm{H}}(x) \varphi(x)-\varphi_{n}(x)\right| & \leqq\left|\int[e(t x)-e(s(t) x)] \Phi_{\mathrm{H}}(t) d t\right| \leqq 4 \omega_{\mathrm{H}}(x) \int\left|\Phi_{\mathrm{H}}(t)\right| d t \\
& \leqq 4 \omega_{\mathrm{H}}(x) h^{-1 / 2}\left|\mathrm{~T}_{n}+\mathrm{H}^{0}+\mathrm{H}^{0}\right|^{1 / 2}\|\varphi\| \leqq 2^{r+2} N_{n}^{1 / 2} \omega_{\mathrm{H}}(x)\|\varphi\|
\end{aligned}
$$


where $N_{n}$ is the number of points in $\mathrm{T}_{n}$. Now let $a_{n}$ be some number with $1<a_{n}<m$. Since $\mathrm{T}_{n}$ is finite, there is a compact set $\mathrm{K}_{n} \subset \Gamma$ such that for $\psi \in E\left(\mathrm{~T}_{n}\right)$ one has $\|\psi\| \leqq a_{n} \sup _{x \in \mathrm{K}_{n}}|\psi(x)|$. We choose $\mathrm{H}=\mathrm{H}_{n}$ so small that for $x \in \mathrm{K}_{n}, \omega_{\mathrm{H}}(x) \leqq\left(a_{n}^{-1} m-1\right) 2^{-r-2} N_{n}^{-1 / 2}$. Using the previous estimate on $\left|k_{\mathrm{H}}(x) \varphi(x)-\varphi_{n}(x)\right|$ we get $\left|\varphi_{n}(x)\right| \leqq a_{n}^{-1} m\|\varphi\|$ for $x \in \mathrm{K}_{n}$ and hence $\left\|\varphi_{n}\right\|$ $\leqq m\|\varphi\|$. Finally, $\left|\varphi(x)-\varphi_{n}(x)\right| \leqq\left\{2^{r+2} N_{n}^{1 / 2}+1\right\} \omega_{\mathrm{H}}(x)\|\varphi\|$ for $\varphi \in E^{\prime}(\mathrm{T})$. This quantity will tend to 0 uniformly on compact sets if $\mathrm{H}_{n} \rightarrow 0$ sufficiently rapidly. Suitable sets $\mathrm{H}_{n}$ can be chosen when the group $\Gamma$ satisfies our requirements.

The above construction furnishes examples of perfect sets $T$ for which somewhat more than spectral synthesis is valid. These sets are, however, quite rarefied. One may suspect that this is due to the crudeness of the estimates which may be the price one has to pay for the freedom of choice in the sets $\mathrm{T}_{n}$. At any rate, if stronger arithmetical requirements are imposed on the sets $\mathrm{T}_{n}$ one can do much better. We shall now suppose that the group $\Gamma$ possesses a family of discrete subgroups $\{\Delta\}$ such that each factor group $\Gamma / \Delta$ is compact and for a given nucleus $N$ in $\hat{\Gamma}$ there is a member $\Delta$ with $\hat{\Gamma}=\Delta^{\perp}+N$. Given $N$ and $\Delta$, let $H$ be chosen as in the conclusion of (5.10). The collection $\{\mathrm{H}\}$ forms what we shall call an arithmetical $J$-net.

6.5. Theorem. Suppose that $\mathrm{T}$ is a closed subset of $\hat{\Gamma}$ and that there exists an arithmetical $J$-net $\{\mathrm{H}\}$ and a collection of points $s=s(\mathrm{H}) \in \hat{\Gamma}$ such that $\left(\mathrm{T}+\mathrm{H}^{0}\right)$ $\cap\left(s+\Delta^{\perp}\right) \subset \mathrm{T}$ for each $\mathrm{H}$. Then spectral synthesis holds for $\mathrm{T}$ in the narrow topology, i.e. given $\varphi \in E^{\prime}(\mathrm{T}), \mathrm{K}$ a compact subset of $\Gamma$, and $\epsilon>0$ there is a trigonometric polynomial $\psi$ with spectrum in $\mathrm{T}$ such that $\|\psi\| \leqq\|\varphi\|$ and $|\varphi(x)-\psi(x)|$ $<\epsilon$ for $x \in \mathrm{K}$.

Proof. We employ a slight modification of (5.13). Set $\varphi_{\mathrm{H}}^{s}(x)$ $=\sum_{y \in \Delta} k_{\mathrm{H}}(x+y) \varphi(x+y) \bar{e}(s y)$. It is obvious that $\varphi_{\mathrm{H}}^{s}=e_{s} \varphi_{\mathrm{H}}^{\prime}$ where $\varphi^{\prime}$ is the function $\bar{e}_{s} \varphi$. Consequently, $\Lambda\left(\varphi_{\mathrm{H}}^{s}\right) \subset s+\left(\mathrm{T}+\mathrm{H}^{0}-s\right) \cap \Delta^{\perp}=\left(\mathrm{T}+\mathrm{H}^{0}\right) \cap\left(s+\Delta^{\perp}\right)$. By our assumption we have $\Lambda\left(\varphi_{\mathrm{H}}^{s}\right) \subset \mathrm{T}$. As in (5.13), $\left\|\varphi_{\mathrm{H}}^{s}\right\| \leqq\|\varphi\|$ and $\left|\varphi(x)-\varphi_{\mathrm{H}}^{s}(x)\right| \leqq\|\varphi\| \omega_{\mathrm{H}}(x)$. Hence $\varphi_{\mathrm{H}}^{s} \rightarrow \varphi$ narrowly. The functions $\varphi_{\mathrm{H}}^{s}$ themselves may not be trigonometric polynomials, but they are at least uniformly almost periodic since $\Gamma / \Delta$ is compact. Thus each $\varphi_{\mathrm{H}}^{s}$ is the uniform limit of trigonometric polynomials with spectra in $\mathrm{T}$, and the theorem is proved.

We wish to make two remarks about this theorem. The first is that one construct sets $\mathrm{T}$ fulfilling the hypotheses which have arbitrary topological or measure-theoretic character. For example, by a Cantor-type procedure one can obtain perfect frontier sets of positive measure in $R^{r}$ which are $S$-sets. The second remark is that if $\mathrm{T}$ is the boundary of a parallelepiped in $R^{r}$ both (6.3) and (6.5) apply. The one says that $\mathrm{T}$ is an $S S$-set; the other that spectral synthesis holds in the narrow topology. These two properties, both of which imply that $\mathbf{T}$ is an $S$-set, seem to involve two entirely different ideas.

EXAmple. Let $\mathrm{T}$ be a "porcupine," i.e. a subset of $R^{3}$ of the form $\mathrm{T}=\mathrm{T}_{1} \cup \mathrm{T}_{2}$ 
where the "body" $T_{1}$ is a locally star-shaped set and the "quills" $T_{2}$ is a finite collection of straight line segments; $\mathrm{T}_{1} \cap \mathrm{T}_{2}=\Sigma$ is a finite set. For each point of $\mathrm{T}_{1} \cap \tilde{\mathrm{T}}_{2}$ we can find a star-shaped subset of $\mathrm{T}$ containing it which is relatively open in $\mathrm{T}$. Each point of $\mathrm{T}_{2} \cap \widetilde{\mathrm{T}}_{1}$ lies on a line segment relatively interior to T. Since star-shaped sets and line segments are $S$-sets, Theorem (6.4) applies to show that $\mathrm{T}$ is an $S$-set.

EXample $\left({ }^{41}\right)$. Suppose $\Gamma=R^{1}$ and $\mathrm{T} \subset \hat{\Gamma}$ is a set constructed by the Cantor method. That is, we have a sequence $b_{1}, b_{2}, \ldots$ of real numbers $b_{n}>1 ; \mathrm{T}_{0}$ is a given interval; $\mathrm{T}_{n}$ consists of $2^{n}$ intervals, and $\mathrm{T}_{n+1}$ is obtained by removing from each subinterval of $\mathrm{T}_{n}$ the middle $b_{n}$ th part; $\mathrm{T}=\bigcap_{n=0}^{\infty} \mathrm{T}_{n}$. $\mathrm{T}$ fulfills the hypotheses of (6.5) if and only if each $b_{n}$ is an integer.

\section{BIBLIOGRAPHY}

\section{N. I. ACHIESER}

1. Approximationstheorie, Berlin, 1953.

R. ARens and I. M. Singer

1. Generalized analytic functions, Trans. Amer. Math. Soc. vol. 81 (1956) pp. 379-393.

S. BANACH

1. Théorie des opérations linéaires, Warsaw-Lwow, 1932.

Arne Beurling

1. Un thêrème sur les fonctions bornées et uniformement continues sur l'axe réel, Acta Math. vol. 77 (1945) pp. 127-136.

2. On the spectral synthesis of bounded functions, Acta Math. vol. 81 (1949) pp. 225-238.

3. Sur les integrales de Fourier absolument convergentes et leur application d une transformation fonctionnelle, Neuvième Congrès des Mathematiciens Scandinaves à Helsingfors, 1938.

4. Sur les spectres des fonctions, Colloques Internationaux du Centre National de la Récherche Scientifique XV; Analyse harmonique, Nancy, 1947.

5. On a closure problem, Ark. Mat. vol. 1 (1951) pp. 301-303.

R. P. BOAs, JR.

1. Entire functions bounded on a line, Duke Math. J. vol. 6 (1940) pp. 148-169.

2. Entire functions, New York, 1954.

S. BOCHNER

1. A theorem on Fourier-Stieltjes integrals, Bull. Amer. Math. Soc. vol. 40 (1934) pp. 271276.

2. Harmonic analysis and the theory of probability, Berkeley-Los Angeles, 1955.

R. C. BuCk

1. Operator algebras and dual spaces, Proc. Amer. Math. Soc. vol. 3 (1952) pp. 681-687.

H. Cartan

1. Sur les fondements de la theorie du potentiel, Bull. Soc. Math. France vol. 69 (1941) pp. 71-96.

H. Cartan and J. Deny

1. Le principe du maximum en theorie du potentiel et la notion de fonction surharmonique, Acta Sci. Math. Szeged. vol. 12 (1950) pp. 81-100.

H. Cartan and R. Godement

1. Theorie de la dualite et analyse harmonique dans les groupes Abeliens localement compacts, Ann. Ecole. Norm. Sup. vol. 64 (1947) pp. 79-99.

(4) The proof of (6.5) for the case in question has been given in HERz [1]. 
G. Choquet

1. Theory of capacities, Ann. Inst. Fourier, Grenoble vol. 5 (1953-1954) pp. 131-295. J. DENY

1. Les potentiels d'energie finie, Acta Math. vol. 83 (1950) pp. 107-183.

YNGVE DOMAR

1. Harmonic analysis based on certain commutative Banach algebras, Acta Math. vol. 96 (1956) pp. 1-66.

W. F. EBERLEIN

1. Abstract ergodic theorems and weak almost periodic functions, Trans. Amer. Math. Soc. vol. 67 (1949) pp. 217-240.

R. Godement

1. Theorèmes Tauberiens et theorie spectrale, Ann. Ecole. Norm. Sup. vol. 64 (1947) pp. 119-138.

C. S. HerZ

1. Spectral synthesis for the Cantor set, Proc. Nat. Acad. Sci. vol. 42 (1956) pp. 42-43.

2. A note on summability methods and spectral analysis, Trans. Amer. Math. Soc. vol. 86 (1957) pp: 506-510.

G. A. HuNT

1. Semi-groups of measures on Lie groups, Trans. Amer. Math. Soc. vol. 81 (1956) pp. 264293.

2. Markoff processes and potentials, Illinois J. Math. vol. 1 (1957) pp. 44-93, 316-369; vol. 2 (1958) pp. 151-213.

S. Kaczmarz and H. Steinhaus

1. Orthogonalreihen, Warsaw-Lwow, 1935.

J.-P. Kahane and R. SALEM

1. Sur les ensembles lineaires ne portant pas de pseudomesures, C. R. Acad. Sci. Paris vol. 243 (1956) pp. 1185-1187.

J. L. KELLEY

1. General topology, New York, 1955.

L. H. Loomis

1. Abstract harmonic analysis, New York, 1953.

G. G. LoRENTz

1. A contribution to the theory of divergent sequences, Acta Math. vol. 80 (1948) pp. 167-190.

P. Malliavin

1. Sur l'impossibilité de la synthèse spectrale sur la droite, C. R. Acad. Sci. Paris vol. 248 (1959) pp. 2155-2157.

R. E. A. C. Paley and N. Wiener

1. Fourier transforms in the complex domain, Amer. Math. Soc. Colloquium Publications, vol. 19, New York, 1934.

M. Plancherel and G. Polya

1. Fonctions entières et intégrales de Fourier multiples, Comm. Math. Helv. vol. 9 (1936) pp. 224-248; vol. 10 (1937) pp. 110-163.

H. PollaRD

1. The harmonic analysis of bounded functions, Duke Math. J. vol. 20 (1953) pp. 499-512.

I. I. Pyatetskir-Shapiro

1. Supplement to the work On the problem of uniqueness of the resolution of functions into trigonometric series (in Russian), Uchenye Zapiski (Moscow University) 165 Mat. VII (1954) pp. 79-97.

H. J. REITER

1. Investigations in harmonic analysis, Trans. Amer. Math. Soc. vol. 73 (1952) pp. 401427. 
2. Contributions to harmonic analysis, I, Acta Math. vol. 96 (1956) pp. 253-263; II, Math. J. Riss Ann. vol. 133 (1957) pp. 298-302; III, J. London Math. Soc. vol. 32 (1957) pp. 477-483.

1. Eléments de calcul differrentiel et théories des distributions sur les groupes Abéliens localement compacts, Acta Math. vol. 89 (1953) pp. 45-105.

I. J. SCHOENBERG

1. A remark on the preceding note by Bochner, Bull. Amer. Math. Soc. vol. 40 (1934) pp. 277-278.

2. Remarks to Maurice Frechet's article, "Sur la deffinition axiomatigue d'une classe d'espaces vectoriels distanciés applicables vectoriellement sur l'espace de Hilbert," Ann. of Math. vol. 36 (1935) pp. 724-732.

3. Metric spaces and positive definite functions, Trans. Amer. Math. Soc. vol. 44 (1938) pp. 522-536.

L. SCHWARTZ

1. Sur une proprieté de synthèse spectrale dans les groupes non compacts, C. R. Acad. Sci. Paris vol. 227 (1948) pp. 424-426.

I. E. SEGAL

1. The group algebra of a locally compact group, Trans. Amer. Math. Soc. vol. 61 (1947) pp. 69-105.

Symposium on Harmonic Analysis and Related Integral Transforms

A. WEIL

Cornell University, 1956, unpublished, but duplicated.

1. L'intégration dans les groupes topologiques et ses applications, Paris, 1938.

N. WIENER

1. The Fourier integral, Cambridge, 1933.

Antoni Zygmund

1. Trigonometric series, Warsaw-Lwow, 1935.

Cornell University, ITHACA, NEW YORK

The Institute for Advanced Study, Princeton, New Jersey 